\title{
ADMISSIBLE DIAGRAMS IN QUANTUM NILPOTENT ALGEBRAS AND COMBINATORIC PROPERTIES OF WEYL GROUPS
}

\author{
ANTOINE MÉRIAUX AND GÉRARD CAUCHON
}

\begin{abstract}
Consider a complex simple Lie algebra $\mathfrak{g}$ of rank $n$. Denote by $\Pi$ a system of simple roots, by $W$ the corresponding Weyl group, consider a reduced expression $w=s_{\alpha_{1}} \circ \cdots \circ s_{\alpha_{t}}$ (each $\alpha_{i} \in \Pi$ ) of some $w \in W$ and call diagram any subset of $\llbracket 1, \ldots, t \rrbracket$. We denote by $U^{+}[w]$ (or $\left.U_{q}^{w}(\mathfrak{g})\right)$ the "quantum nilpotent" algebra as defined by Jantzen in 1996

We prove (theorem 5.3.1) that the positive diagrams naturally associated with the positive subexpressions (of the reduced expression of $w$ chosen above) in the sense of R. Marsh and K. Rietsch (or equivalently the subexpressions without defect in the sense of V. Deodhar), coincide with the admissible diagrams constructed by G. Cauchon which describe the natural stratification of $\operatorname{Spec}\left(U^{+}[w]\right)$.

This theorem implies in particular (corollaries 5.3.1 and 5.3.2):

(1) The map $\zeta: \Delta=\left\{j_{1}<\cdots<j_{s}\right\} \mapsto u=s_{\alpha_{j_{1}}} \circ \cdots \circ s_{\alpha_{j_{s}}}$ is a bijection from the set of admissible diagrams onto the set $\{u \in W \mid u \leq w\}$.

(2) For each admissible diagram $\Delta=\left\{j_{1}<\cdots<j_{s}\right\}, s_{\alpha_{j_{1}}} \circ \cdots \circ s_{\alpha_{j_{s}}}$ is a reduced expression of $u=\zeta(\Delta)$.

(3) The map $\zeta^{\prime}: \Delta=\left\{j_{1}<\cdots<j_{s}\right\} \mapsto u^{\prime}=s_{\alpha_{j_{s}}} \circ \cdots \circ s_{\alpha_{j_{1}}}$ is a bijection from the set of admissible diagrams onto the set $\left\{u \in W \mid u \leq v=w^{-1}\right\}$.

(4) For each admissible diagram $\Delta=\left\{j_{1}<\cdots<j_{s}\right\}, s_{\alpha_{j_{s}}} \circ \cdots \circ s_{\alpha_{j_{1}}}$ is a reduced expression of $u^{\prime}=\zeta^{\prime}(\Delta)$.

If the Lie algebra $\mathfrak{g}$ is of type $A_{n}$ and $w$ is chosen in order that $U^{+}[w]$ is the algebra of quantum matrices $O_{q}\left(M_{p, m}(k)\right)$ with $m=n-p+1$ (see section 2.1), then, the admissible diagrams are the I-diagrams in the sense of A. Postnikov (http://arxiv.org/abs/math/0609764). In this particular case, the assertions 3 and 4 have also been proved (with quite different methods) by A. Postnikov and by T. Lam and L. Williams.
\end{abstract}

\section{INTRODUCTION}

Given a complex simple Lie algebra $\mathfrak{g}$ of rank $n$, denote by $\Pi$ a system of simple roots, by $W$ the corresponding Weyl group, and consider a reduced expression $w=s_{\alpha_{1}} \circ \cdots \circ s_{\alpha_{t}}$ of some $w \in W$.

Denote by $U^{+}[w]$ (or $\left.U_{q}^{w}(\mathfrak{g})\right)$ the "quantum nilpotent" algebra associated to $w$ as in [12] and by $X_{1}, \ldots, X_{t}$ the canonical generators of $U^{+}[w]$ associated with this chosen reduced expression of $w$ (see section 2.1 for more details).

The natural action of the torus (namely the multiplicative group of the $K_{\lambda}$, where $\lambda$ runs through the additive group generated by the roots system; see 22,

Received by the editors July 24, 2008 and, in revised form, March 24, 2010.

2010 Mathematics Subject Classification. Primary 17B37; Secondary 16T20.

(C)2010 American Mathematical Society Reverts to public domain 28 years from publication 
Chapter 4]) on the prime spectrum $\operatorname{Spec}\left(U^{+}[w]\right)$ induces a finite stratification which is completely described by the admissible diagrams (also called Cauchon diagrams) which are some particular subsets of $\llbracket 1, \ldots, t \rrbracket$ (see [4]). Those admissible diagrams are very closely connected with the quantum commutation rules satisfied by the canonical generators. For example, in the $A_{n}$ type, one can choose $w$ such that $U^{+}[w]$ is a quantum matrix algebra

$$
O_{q}\left(M_{p, m}(k)\right)=k\left\langle X_{i, j}\right\rangle(1 \leq i \leq p, 1 \leq j \leq m),
$$

and we know [5] that the commutation relations

$$
X_{u, v} X_{i, j}=X_{i, j} X_{u, v}-\left(q-q^{-1}\right) X_{i, v} X_{u, j}(i<u, j<v)
$$

determine the shape of admissible diagrams by the following observation:

If $\mathcal{P}$ is any completely prime ideal of $O_{q}\left(M_{p, m}(k)\right)$, we have immediately

$$
X_{u, v} \in \mathcal{P} \Rightarrow\left(X_{i, v} \in \mathcal{P} \text { or } X_{u, j} \in \mathcal{P}\right) .
$$

This implies $\llbracket 5]$ that $\Delta \subset \llbracket 1, \ldots, p \rrbracket \times \llbracket 1, \ldots, m \rrbracket$ (the set of indexes) is an admissible diagram if and only if, for each $i, j, u, v$ as above, we have:

$$
(u, v) \in \Delta \Rightarrow((i, v) \in \Delta \text { or }(u, j) \in \Delta) .
$$

This means that the admissible diagrams are the unions of truncated rows and columns, namely the I-diagrams in the sense of A. Postnikov (see [20]).

So, we see in this example that admissible diagrams are quantum objects since, in the nonquantum case (when $q=1$ ), the formulas $\left(*\right.$ ) become $X_{u, v} X_{i, j}=X_{i, j} X_{u, v}$, so that observation $(* *)$ is no longer valid and the admissible diagrams become invisible.

On the other hand, R. Marsh and K. Rietsch [18] defined the notion of positive subexpression of the reduced expression of $w$ considered above. These positive subexpressions are defined by particular subsets of $\llbracket 1, \ldots, t \rrbracket$ that we call the positive diagrams. R. Marsh and K. Rietsch proved in [18] that they are in one-to-one natural correspondence with the elements of the Weyl group which are smaller or equal to $w$ (for the Bruhat order). In this paper, we prove (theorem 5.3. 1) that the positive diagrams coincide with the admissible diagrams, which can be interpreted saying that R. Marsh and K. Rietsch's positive subexpressions are quantum objects.

In particular, this implies (corollary 5.3. 1):

(1) The map $\zeta: \Delta=\left\{j_{1}<\cdots<j_{s}\right\} \mapsto u=s_{\alpha_{j_{1}}} \circ \cdots \circ s_{\alpha_{j_{s}}}$ is a bijection from the set of admissible diagrams onto the set $\{u \in W \mid u \leq w\}$.

(2) Consider an admissible diagram $\Delta=\left\{j_{1}<\cdots<j_{s}\right\}$ and some integer $i \in \llbracket 1, \ldots, t \rrbracket$. Set $\Delta \cap \llbracket i+1, \ldots, t \rrbracket=\left\{j_{c}, \ldots, j_{s}\right\}(1 \leq c \leq s)$. Then the expression $s_{\alpha_{i}} \circ s_{\alpha_{j_{c}}} \circ \cdots \circ s_{\alpha_{j_{s}}}$ is reduced. In particular, $s_{\alpha_{j_{1}}} \circ \cdots \circ s_{\alpha_{j_{s}}}$ is a reduced expression of $\zeta(\Delta)$,

(3) Consider some $u \in W$ with $u \leq w$. Then, the only admissible diagram $\Delta$ such that $\zeta(\Delta)=u$ is recursively defined as follows:

- $1 \in \Delta \Leftrightarrow l\left(s_{\alpha_{1}} \circ u\right)=l(u)-1 \Leftrightarrow u^{-1}\left(\alpha_{1}\right)$ is a negative root;

- consider some integer $i \in \llbracket 1, \ldots, t-1 \rrbracket$, assume that $\Delta \cap \llbracket 1, \ldots, i \rrbracket=$ $\left\{j_{1}, \ldots, j_{d}\right\}$ and set $u_{i}=s_{\alpha_{j_{d}}} \circ \cdots \circ s_{\alpha_{j_{2}}} \circ s_{\alpha_{j_{1}}} \circ u\left(u_{i}=u\right.$ if $\left.\Delta \cap \llbracket 1, \ldots, i \rrbracket=\emptyset\right)$.

Then $(i+1 \in \Delta) \Leftrightarrow\left(l\left(s_{\alpha_{i+1}} \circ u_{i}\right)=l\left(u_{i}\right)-1\right) \Leftrightarrow\left(u_{i}^{-1}\left(\alpha_{i+1}\right)\right.$ is a negative root).

It also implies (corollary 5.3. 2): 
(1) The map $\zeta^{\prime}: \Delta=\left\{j_{1}<\cdots<j_{s}\right\} \mapsto u^{\prime}=s_{\alpha_{j_{s}}} \circ \cdots \circ s_{\alpha_{j_{1}}}$ is a bijection from the set of admissible diagrams onto the set $\left\{u \in W \mid u \leq v=w^{-1}\right\}$.

(2) Consider an admissible diagram $\Delta=\left\{j_{1}<\cdots<j_{s}\right\}$ and some integer $i \in \llbracket 1, \ldots, t \rrbracket$. Set $\Delta \cap \llbracket i+1, \ldots, t \rrbracket=\left\{j_{c}, \ldots, j_{s}\right\}(1 \leq c \leq s)$. Then the expression $s_{\alpha_{j_{s}}} \circ \cdots \circ s_{\alpha_{j_{c}}} \circ s_{\alpha_{i}}$ is reduced. In particular, $s_{\alpha_{j_{s}}} \circ \cdots \circ s_{\alpha_{j_{1}}}$ is a reduced expression of $\zeta^{\prime}(\Delta)$.

In the particular case $U^{+}[w]=O_{q}\left(M_{p, m}(k)\right)$ mentioned above, this last result gives back a theorem of A. Postnikov [20] and a theorem of T. Lam and L. Williams [17.

In the general case, let us denote by $\Delta \leftrightarrow w^{\Delta}$ the one-to-one correspondence constructed by R. Marsh and K. Rietsch [18 between the positive diagrams and the elements of the Weyl group which are smaller or equal to $w$.

Let us also denote by $\Delta \leftrightarrow \mathcal{P}_{\Delta}$ the one-to-one correspondence constructed by G. Cauchon $\left[4\right.$ between the admissible diagrams and the prime ideals of $U^{+}[w]$ which are invariant under the torus action.

By theorem 5.3.1 of this paper, it turns out that there exists a natural one-to-one correspondence

$$
w^{\Delta} \leftrightarrow \mathcal{P}_{\Delta}
$$

between the elements of the Weyl group which are smaller or equal to $w$ and the prime ideals of $U^{+}[w]$ which are invariant under the torus action.

Let us recall that, in the particular case $U^{+}[w]=O_{q}\left(M_{p, m}(k)\right)$ mentioned above, S. Launois 14 has constructed (with quite different methods) such a one-to-one correspondence which, moreover, preserves the ordering (where the Weyl group is provided with the Bruhat order and the spectrum $\operatorname{Spec}\left(O_{q}\left(M_{p, m}(k)\right)\right)$ is provided with the inclusion order). This leads us to ask the following questions (unsolved at the moment):

- Question 1. Does this Launois correspondence coincide with ours in the particular case $U^{+}[w]=O_{q}\left(M_{p, m}(k)\right)$ ?

- Question 2. Does our correspondence $w^{\Delta} \leftrightarrow \mathcal{P}_{\Delta}$ preserve the ordering in the general case (where the Weyl group is provided with the Bruhat order and the spectrum $\operatorname{Spec}\left(U^{+}[w]\right)$ is provided with the inclusion order)?

(A positive answer would supply the Bruhat order with a nice quantum interpretation.)

\section{BACKGROUND ON WEYL GROUPS}

Following J. C. Jantzen ([12]), we use the following conventions throughout this paper:

- $\mathfrak{g}$ is a complex simple Lie algebra.

- $\Phi$ is the (irreducible) root system of $\mathfrak{g}$ with respect to a fixed Cartan subalgebra.

- $\Pi$ is a fixed basis of $\Phi, \Phi^{+}$denotes the subset of positive roots, and we set $n=|\Pi|, \quad \mathrm{N}=\left|\Phi^{+}\right|(1 \leq n \leq N)$.

- $W$ is the Weyl group of $\Phi$ and (, ) is the unique scalar product on the real vector space $V$ generated by $\Phi$, such that $\left.\|\beta\|^{2}=2(\|\beta\|:=\sqrt{(\beta, \beta})\right)$ for all short roots $\beta$ in $\Phi$.

- For any $\beta$ in $\Phi$, we set $d_{\beta}=\frac{\|\beta\|^{2}}{2}, \beta^{\vee}=\frac{\beta}{d_{\beta}}$, and $s_{\beta}$ denotes the reflection with respect to $\beta\left(s_{\beta}(x)=x-\left(\beta^{\vee}, x\right) \beta\right.$ for any $x$ in $\left.V\right)$. 
- $k$ is a field with $\operatorname{char}(k) \neq 2$ and, in addition, $\operatorname{char}(k) \neq 3$ if $\Phi$ has type $G_{2}$. - $q \in k^{*}:=k \backslash\{0\}, q$ is not a root of unity.

- The $k$-algebra $U_{q}(\mathfrak{g})$ and its canonical generators $E_{\alpha}, F_{\alpha}, K_{\alpha}^{ \pm 1}(\alpha \in \Pi)$ are defined as in [12]. We denote by $U^{+}\left(\right.$or $\left.U_{q}^{+}(\mathfrak{g})\right)$ the subalgebra generated by the $E_{\alpha}(\alpha \in \Pi)$.

- Denote by $\mathbb{Z} \Pi$ the root lattice. The algebra $U_{q}(\mathfrak{g})$ is $\mathbb{Z} \Pi$-graded and, if $\alpha$ in $\Pi, \operatorname{deg}\left(E_{\alpha}\right)=\alpha, \operatorname{deg}\left(F_{\alpha}\right)=-\alpha, \operatorname{deg}\left(K_{\alpha}^{ \pm 1}\right)=0$.

- For any $\rho=\sum_{\alpha \in \Pi} m_{\alpha} \alpha\left(m_{\alpha} \in \mathbb{Z}\right)$ in the root lattice $\mathbb{Z} \Pi$, we set $K_{\rho}=$ $\prod_{\alpha \in \Pi} K_{\alpha}^{m_{\alpha}}$. The multiplicative group $T=\left\{K_{\rho} \mid \rho \in \mathbb{Z} \Pi\right\}$ is called the Torus (of $\left.U_{q}(\mathfrak{g})\right)$. This group acts on the algebra $U_{q}(\mathfrak{g})$ by

$$
K_{\rho} . u=K_{\rho}^{-1} u K_{\rho}\left(\forall u \in U_{q}(\mathfrak{g})\right) .
$$

In particular, for any homogeneous element $u$ of $U_{q}(\mathfrak{g})$ with degree $\operatorname{deg}(u)$ $=\gamma \in \mathbb{Z} \Pi$, we have $K_{\rho} \cdot u=q^{-(\rho, \gamma)} u$.

2.1. Quantum algebras $U^{+}[w]$. Consider any $w \in W$, set $t=l(w)$ and consider a reduced expression

$$
w=s_{\alpha_{1}} \circ \cdots \circ s_{\alpha_{t}} \quad\left(\alpha_{i} \in \Pi \text { for } 1 \leq i \leq t\right) .
$$

It is well known that

$$
\beta_{1}=\alpha_{1}, \beta_{2}=s_{\alpha_{1}}\left(\alpha_{2}\right), \ldots, \beta_{t}=s_{\alpha_{1}} \ldots s_{\alpha_{t-1}}\left(\alpha_{t}\right)
$$

are distinct positive roots and that the set $\left\{\beta_{1}, \ldots, \beta_{t}\right\}$ does not depend on the reduced expression (11) of $w$. For any $\alpha \in \Pi$, define the braid automorphism $T_{\alpha}$ of the algebra $U_{q}(\mathfrak{g})$ as in $(12$, p. 153), and set

$$
X_{\beta_{1}}=E_{\alpha_{1}}, X_{\beta_{2}}=T_{\alpha_{1}}\left(E_{\alpha_{2}}\right), \ldots, X_{\beta_{t}}=T_{\alpha_{1}} \ldots T_{\alpha_{t-1}}\left(E_{\alpha_{t}}\right) .
$$

The following results are classical ([12], chapter 8):

- $X_{\beta_{1}}, \ldots, X_{\beta_{t}}$ are all in $U^{+}$. Each $X_{\beta_{i}}(1 \leq i \leq t)$ is homogeneous of degree $\operatorname{deg}\left(X_{\beta_{i}}\right)=\beta_{i}$.

- We denote by $U^{+}[w]\left(\right.$ or $\left.U_{q}^{w}(\mathfrak{g})\right)$ the subalgebra of $U^{+}$generated by $X_{\beta_{1}}$, $\ldots, X_{\beta_{t}}$. This algebra does not depend on the above reduced expression (11) of $w$ (although the variables $X_{\beta_{1}}, \ldots, X_{\beta_{t}}$ depend on (11)).

- The ordered monomials $X^{\underline{a}}:=X_{\beta_{1}}^{a_{1}}, \ldots, X_{\beta_{t}}^{a_{t}}, \underline{a}=\left(a_{1}, \ldots, a_{t}\right) \in \mathbb{N}^{t}$, are a basis of $U^{+}[w]$ (as a $k$-vector space).

- Since the above generators $X_{\beta_{i}}$ are homogeneous, the $\mathbb{Z} \Pi$-graduation of $U_{q}(\mathfrak{g})$ induces a $\mathbb{Z} \Pi$-graduation of $U^{+}[w]$ and the action of the Torus $T$ on $U_{q}(\mathfrak{g})$ induces, by restriction, an action of $T$ on $U^{+}[w]$.

- If $1 \leq i<j \leq t$, we have the following straightening formula due to Levendorskii and Soibelman:

$$
\begin{gathered}
X_{\beta_{j}} X_{\beta_{i}}-q^{-\left(\beta_{i}, \beta_{j}\right)} X_{\beta_{i}} X_{\beta_{j}}=P_{j, i}, \\
P_{j, i}=\sum_{\underline{a}=\left(a_{i+1}, \ldots, a_{j-1}\right)} c_{\underline{a}} X_{\beta_{i+1}}^{a_{i+1}} \ldots X_{\beta_{j-1}}^{a_{j-1}},
\end{gathered}
$$

with $\underline{a} \in \mathbb{N}^{j-i-1}, c_{\underline{a}} \in k$, and $c_{\underline{a}} \neq 0$ for only finitely many $\underline{a}$. $P_{j, i}$ is homogeneous with degree $\beta_{i}+\beta_{j}$ so that, if $j=i+1$, we have $P_{j, i}=0$.

The reader will observe a little difference between those formulas and the original Levendorskii-Soibelman one ([16], prop. 5.5.2.), in which the left member of (2) is $X_{\beta_{j}} X_{\beta_{i}}-q^{\left(\beta_{i}, \beta_{j}\right)} X_{\beta_{i}} X_{\beta_{j}}$. The reason is that Levendorskii 
and Soibelman use a version of the quantum group $U_{q}(\mathfrak{g})$ which slightly differs from ours. Under our conventions, a direct proof of formulas (2) and (3) is given in [19].

- When $w=w_{0}$, we have $t=N,\left\{\beta_{1}, \ldots, \beta_{t}\right\}=\Phi^{+}$and $U^{+}[w]=U^{+}$.

An example. Assume, for sake of simplicity, that $k=\mathbb{C}$ is the complex number field, that $\mathfrak{g}$ has type $A_{n}$ with $n \geq 3$ and that the simple roots $\epsilon_{1}, \ldots, \epsilon_{n}$ are ordered such that the Dynkin diagram is

$$
\epsilon_{1}-\epsilon_{2}-\epsilon_{3}-\cdots-\epsilon_{n-1}-\epsilon_{n} .
$$

Consider the following particular reduced expression of the longest element in $W$ :

(4) $w_{0}=s_{\epsilon_{1}} \circ\left(s_{\epsilon_{2}} \circ s_{\epsilon_{1}}\right) \circ \cdots \circ\left(s_{\epsilon_{j}} \circ s_{\epsilon_{j-1}} \circ \cdots \circ s_{\epsilon_{1}}\right) \circ \cdots \circ\left(s_{\epsilon_{n}} \circ s_{\epsilon_{n-1}} \circ \cdots \circ s_{\epsilon_{1}}\right)$.

Denote by $Y_{1,2}, Y_{1,3}, Y_{2,3}, \ldots, Y_{1, j+1}, Y_{2, j+1}, \ldots, Y_{j, j+1}, \ldots, Y_{1, n+1}, Y_{2, n+1}, \ldots$, $Y_{n, n+1}$ the canonical generators of $U^{+}$with respect to this reduced decomposition and observe that:

Lemma 2.1.1. (1) $Y_{j, j+1}=E_{\alpha_{j}}$ for $1 \leq j \leq n$.

(2) $Y_{i, j+1}=Y_{i, j} Y_{j, j+1}-q^{-1} Y_{j, j+1} Y_{i, j}$ for $1 \leq i<j \leq n$.

Proof. (1) This results from the equality

$$
s_{\epsilon_{1}} \circ\left(s_{\epsilon_{2}} \circ s_{\epsilon_{1}}\right) \circ \cdots \circ\left(s_{\epsilon_{j}} \circ s_{\epsilon_{j-1}} \circ \cdots \circ s_{\epsilon_{2}}\right)\left(\epsilon_{1}\right)=\epsilon_{j} .
$$

(2) Recall (see section 2.1) that

$$
\begin{aligned}
Y_{i, j+1}= & T_{\epsilon_{1}} \circ\left(T_{\epsilon_{2}} \circ T_{\epsilon_{1}}\right) \circ \cdots \circ\left(T_{\epsilon_{j-1}}\right. \\
& \left.\circ T_{\epsilon_{j-2}} \circ \cdots \circ T_{\epsilon_{1}}\right) \circ\left(T_{\epsilon_{j}} \circ T_{\epsilon_{j-1}} \circ \cdots \circ T_{\epsilon_{j+2-i}}\right)\left(E_{\epsilon_{j+1-i}}\right) \quad(i>1) .
\end{aligned}
$$

(In the case $i=1$, this formula becomes $Y_{1, j+1}=T_{\epsilon_{1}} \circ\left(T_{\epsilon_{2}} \circ T_{\epsilon_{1}}\right) \circ \cdots \circ$ $\left(T_{\epsilon_{j-1}} \circ T_{\epsilon_{j-2}} \circ \ldots \circ T_{\epsilon_{1}}\right)\left(E_{\epsilon_{j}}\right)$.)

Assume $i>1$. Since $\left\{\epsilon_{j-i}, \ldots, \epsilon_{1}\right\} \perp\left\{\epsilon_{j}, \ldots, \epsilon_{j+2-i}\right\}$ and $\left\{\epsilon_{j-i-1}, \ldots, \epsilon_{1}\right\}$ $\perp \epsilon_{j+1-i}, Y_{i, j+1}$ is equal to

$$
\begin{aligned}
T_{\epsilon_{1}} \circ\left(T_{\epsilon_{2}} \circ T_{\epsilon_{1}}\right) & \circ \cdots \circ\left(T_{\epsilon_{j-1}} \circ T_{\epsilon_{j-2}} \circ \cdots \circ T_{\epsilon_{j+1-i}}\right) \\
& \circ\left(T_{\epsilon_{j}} \circ T_{\epsilon_{j-1}} \circ \cdots \circ T_{\epsilon_{j+2-i}}\right) \circ T_{\epsilon_{j-i}}\left(E_{\epsilon_{j+1-i}}\right)
\end{aligned}
$$

with, by [12,

$$
T_{\epsilon_{j-i}}\left(E_{\epsilon_{j+1-i}}\right)=E_{\epsilon_{j-i}} E_{\epsilon_{j+1-i}}-q^{-1} E_{\epsilon_{j+1-i}} E_{\epsilon_{j-i}} .
$$

Moreover, we have

$$
\begin{aligned}
& s_{\epsilon_{1}} \circ\left(s_{\epsilon_{2}} \circ s_{\epsilon_{1}}\right) \circ \cdots \circ\left(s_{\epsilon_{j-1}} \circ s_{\epsilon_{j-2}} \circ \cdots \circ s_{\epsilon_{j+1-i}}\right) \circ\left(s_{\epsilon_{j}} \circ s_{\epsilon_{j-1}} \circ \cdots \circ s_{\epsilon_{j+2-i}}\right)\left(\epsilon_{j+1-i}\right) \\
& \quad=s_{\epsilon_{1}} \circ\left(s_{\epsilon_{2}} \circ s_{\epsilon_{1}}\right) \circ \cdots \circ\left(s_{\epsilon_{j-1}} \circ s_{\epsilon_{j-2}} \circ \cdots \circ s_{\epsilon_{j+1-i}}\right)\left(\epsilon_{j+1-i}+\epsilon_{j+2-i}+\cdots+\epsilon_{j}\right) \\
& \quad=s_{\epsilon_{1}} \circ\left(s_{\epsilon_{2}} \circ s_{\epsilon_{1}}\right) \circ \cdots \circ\left(s_{\epsilon_{j-2}} \circ s_{\epsilon_{j-3}} \circ \ldots \circ s_{\epsilon_{1}}\right)\left(\epsilon_{j}\right)=\epsilon_{j} .
\end{aligned}
$$

This implies that

$$
\begin{aligned}
T_{\epsilon_{1}} & \circ\left(T_{\epsilon_{2}} \circ T_{\epsilon_{1}}\right) \circ \cdots \circ\left(T_{\epsilon_{j-1}} \circ T_{\epsilon_{j-2}} \circ \cdots \circ T_{\epsilon_{j+1-i}}\right) \\
& \circ\left(T_{\epsilon_{j}} \circ T_{\epsilon_{j-1}} \circ \cdots \circ T_{\epsilon_{j+2-i}}\right)\left(E_{\epsilon_{j+1-i}}\right)=E_{\epsilon_{j}}=Y_{j, j+1} .
\end{aligned}
$$

Since $\left\{\epsilon_{j}, \epsilon_{j-1}, \ldots, \epsilon_{j+2-i}\right\} \perp \epsilon_{j-i}$, we have

$$
\begin{aligned}
& T_{\epsilon_{1}} \circ\left(T_{\epsilon_{2}} \circ T_{\epsilon_{1}}\right) \circ \cdots \circ\left(T_{\epsilon_{j-1}} \circ T_{\epsilon_{j-2}} \circ \cdots \circ T_{\epsilon_{j+1-i}}\right) \circ\left(T_{\epsilon_{j}} \circ T_{\epsilon_{j-1}} \circ \cdots \circ T_{\epsilon_{j+2-i}}\right)\left(E_{\epsilon_{j-i}}\right) \\
& \quad=T_{\epsilon_{1}} \circ\left(T_{\epsilon_{2}} \circ T_{\epsilon_{1}}\right) \circ \cdots \circ\left(T_{\epsilon_{j-1}} \circ T_{\epsilon_{j-2}} \circ \cdots \circ T_{\epsilon_{j+1-i}}\right)\left(E_{\epsilon_{j-i}}\right)=Y_{i, j} .
\end{aligned}
$$

So, $Y_{i, j+1}=Y_{i, j} Y_{j, j+1}-q^{-1} Y_{j, j+1} Y_{i, j}$. 
In the case $i=1$, we get

$$
\begin{aligned}
Y_{1, j+1} & =T_{\epsilon_{1}} \circ\left(T_{\epsilon_{2}} \circ T_{\epsilon_{1}}\right) \circ \cdots \circ\left(T_{\epsilon_{j-1}} \circ T_{\epsilon_{j-2}} \circ \cdots \circ T_{\epsilon_{1}}\right)\left(E_{\epsilon_{j}}\right) \\
& =T_{\epsilon_{1}} \circ\left(T_{\epsilon_{2}} \circ T_{\epsilon_{1}}\right) \circ \cdots \circ\left(T_{\epsilon_{j-2}} \circ T_{\epsilon_{j-3}} \circ \cdots \circ T_{\epsilon_{1}}\right) \circ T_{\epsilon_{j-1}}\left(E_{\epsilon_{j}}\right)
\end{aligned}
$$

with, by $[12$,

$$
T_{\epsilon_{j-1}}\left(E_{\epsilon_{j}}\right)=E_{\epsilon_{j-1}} E_{\epsilon_{j}}-q^{-1} E_{\epsilon_{j}} E_{\epsilon_{j-1}} .
$$

We observe that

$T_{\epsilon_{1}} \circ\left(T_{\epsilon_{2}} \circ T_{\epsilon_{1}}\right) \circ \cdots \circ\left(T_{\epsilon_{j-2}} \circ T_{\epsilon_{j-3}} \circ \cdots \circ T_{\epsilon_{1}}\right)\left(E_{\epsilon_{j}}\right)=E_{\epsilon_{j}}=Y_{j, j+1}$,

and that

$$
T_{\epsilon_{1}} \circ\left(T_{\epsilon_{2}} \circ T_{\epsilon_{1}}\right) \circ \cdots \circ\left(T_{\epsilon_{j-2}} \circ T_{\epsilon_{j-3}} \circ \cdots \circ T_{\epsilon_{1}}\right)\left(E_{\epsilon_{j-1}}\right)=Y_{1, j} .
$$

So, $Y_{1, j+1}=Y_{1, j} Y_{j, j+1}-q^{-1} Y_{j, j+1} Y_{1, j}$.

Denote by $v$ a square root of $q$ and, as in [1, denote by $e_{i, j}$ the generators of $U^{+}$constructed by H. Yamane 22. We know that:

- $e_{j, j+1}=E_{\epsilon_{j}}$ for $1 \leq j \leq n$.

- $e_{i, j+1}=v e_{i, j} e_{j, j+1}-v^{-1} e_{j, j+1} e_{i, j}$ for $1 \leq i<j \leq n$.

This implies:

- $v^{i-j} e_{i, j+1}=\left(v^{i-j+1} e_{i, j}\right) e_{j, j+1}-e_{j, j+1} q^{-1}\left(v^{i-j+1} e_{i, j}\right)$ for $1 \leq i<j \leq n$ and, from lemma 2.1. 1, we deduce (by induction on $j-i$ ):

Lemma 2.1.2. $Y_{i, j+1}=v^{i-j} e_{i, j+1}$ for $1 \leq i \leq j \leq n$.

Now, consider an integer $p$ with $1<p<n$ and set

(5) $w=\left(s_{\epsilon_{p}} \circ s_{\epsilon_{p-1}} \circ \cdots \circ s_{\epsilon_{1}}\right) \circ\left(s_{\epsilon_{p+1}} \circ s_{\epsilon_{p}} \circ \cdots \circ s_{\epsilon_{2}}\right) \circ \cdots \circ\left(s_{\epsilon_{n}} \circ s_{\epsilon_{n-1}} \circ \cdots \circ s_{\epsilon_{n-p+1}}\right)$.

If $p<j<n$, we observe that

$$
\left\{\epsilon_{j-p}, \ldots, \epsilon_{1}\right\} \perp\left\{\epsilon_{j+1}, \ldots, \epsilon_{j+1-p+1}, \ldots, \epsilon_{n}, \ldots, \epsilon_{n-p+1}\right\} .
$$

This implies that

$$
w_{0}=w_{1} \circ w \circ w_{2}
$$

with

$$
\begin{gathered}
w_{1}=s_{\epsilon_{1}} \circ\left(s_{\epsilon_{2}} \circ s_{\epsilon_{1}}\right) \circ \cdots \circ\left(s_{\epsilon_{p-1}} \circ s_{\epsilon_{p-2}} \circ \cdots \circ s_{\epsilon_{1}}\right), \\
w_{2}=s_{\epsilon_{1}} \circ\left(s_{\epsilon_{2}} \circ s_{\epsilon_{1}}\right) \circ \cdots \circ\left(s_{\epsilon_{n-p}} \circ s_{\epsilon_{n-p-1}} \circ \cdots \circ s_{\epsilon_{1}}\right) .
\end{gathered}
$$

If $d, d_{1}, d_{2}$ denote the number of simple reflections which appear respectively in the expressions of $w, w_{1}, w_{2}$ given above, we observe that $d+d_{1}+d_{2}$ is equal to the length of $w_{0}$. This implies:

Lemma 2.1.3. (1) (5) is a reduced expression of $w$.

(2) If $p \leq j \leq n$, then

$T_{w_{1}} \circ\left(T_{\epsilon_{p}} \circ T_{\epsilon_{p-1}} \circ \cdots \circ T_{\epsilon_{1}}\right) \circ \cdots \circ\left(T_{\epsilon_{j-1}} \circ T_{\epsilon_{j-2}} \circ \cdots \circ T_{\epsilon_{j-p+1}}\right)\left(E_{\epsilon_{j}}\right)=Y_{j, j+1}$.

(1) If $p \leq j \leq n$ and $1 \leq i \leq p$, then

$$
T_{w_{1}} \circ\left(T_{\epsilon_{p}} \circ T_{\epsilon_{p-1}} \circ \cdots \circ T_{\epsilon_{1}}\right) \circ \cdots \circ\left(T_{\epsilon_{j}} \circ T_{\epsilon_{j-1}} \circ \cdots \circ T_{\epsilon_{j-i+2}}\right)\left(E_{\epsilon_{j-i+1}}\right)=Y_{i, j+1} \text {. }
$$


This implies that the canonical generators $X_{i, j+1}$ of $U^{+}[w]$ satisfy

$$
X_{i, j+1}=T_{w_{1}}^{-1}\left(Y_{i, j+1}\right)
$$

for $p \leq j \leq n$ and $1 \leq i \leq p$.

Since the Yamane generators $e_{i, j+1}(p \leq j \leq n, 1 \leq i \leq p)$ satisfy the commutation relations of quantum matrices, it follows from lemma 2.1. 2 that the generators $Y_{i, j+1}$ satisfy the same property. Now, as $T_{w_{1}}^{-1}$ is an automorphism of $U_{q}(\mathfrak{g})$ and as the ordered monomials in the variables $X_{i, j+1}$ (where the order on these variables is defined by the inverse lexicographic order on the indexes $(i, j+1)$ ) are a basis of $U^{+}[w]$, we conclude that

Proposition 2.1.1. $U^{+}[w]$ is the algebra $O_{q}\left(M_{p, m}(k)\right)$ with $m=n-p+1$ and with canonical generators the variables $X_{i, j+1}(p \leq j \leq n, 1 \leq i \leq p)$.

2.2. Diagrams. We assume that $w, t$, the simple roots $\alpha_{i}$, and the positive roots $\beta_{i}(1 \leq i \leq t)$, are defined as in section 2.1 , and we set

$$
v=w^{-1}=s_{\alpha_{t}} \circ \cdots \circ s_{\alpha_{1}} .
$$

Definition 2.2.1. A diagram with respect to (1) is any subset $\Delta$ of $\llbracket 1, \ldots, t \rrbracket$. (If there is no possible ambiguity, we omit to make precise "with respect to (1)".)

In the following, we sometimes also omit the symbol o in the composition of maps.

Consider a diagram $\Delta$. For any $i \in \llbracket 1, \ldots, t \rrbracket$, we set

$$
s_{\alpha_{i}}^{\Delta}=\left\{\begin{array}{cl}
s_{\alpha_{i}} & \text { if } i \in \Delta \\
I d & \text { if } i \notin \Delta
\end{array}\right\}
$$

and we denote

- $w^{\Delta}=s_{\alpha_{1}}^{\Delta} \ldots s_{\alpha_{t}}^{\Delta}$,

- $v^{\Delta}=\left(w^{\Delta}\right)^{-1}=s_{\alpha_{t}}^{\Delta} \ldots s_{\alpha_{1}}^{\Delta}$,

- for any $i \in \llbracket 1, \ldots, t, t+1 \rrbracket, w_{i}^{\Delta}=s_{\alpha_{i}}^{\Delta} \ldots s_{\alpha_{t}}^{\Delta}\left(w_{1}^{\Delta}=w^{\Delta}, w_{t+1}^{\Delta}=I d\right)$,

- for any $i \in \llbracket 0,1, \ldots, t \rrbracket, v_{i}^{\Delta}=\left(w_{t-i+1}^{\Delta}\right)^{-1}=s_{\alpha_{t}}^{\Delta} \ldots s_{\alpha_{t-i+2}}^{\Delta} s_{\alpha_{t-i+1}}^{\Delta}\left(v_{0}^{\Delta}=\right.$ $\left.\left(w_{t+1}^{\Delta}\right)^{-1}=I d, v_{t}^{\Delta}=v^{\Delta}\right)$.

Remark (the case of example 2.1). Assume we are in the situation of example 2.1, so that

$$
\begin{aligned}
& w=s_{\alpha_{1}} \circ s_{\alpha_{2}} \circ \cdots \circ s_{\alpha_{t}}=\left(s_{\epsilon_{p}} \circ s_{\epsilon_{p-1}} \circ \cdots \circ s_{\epsilon_{1}}\right) \\
& \quad \circ\left(s_{\epsilon_{p+1}} \circ s_{\epsilon_{p}} \circ \cdots \circ s_{\epsilon_{2}}\right) \circ \cdots \circ\left(s_{\epsilon_{n}} \circ s_{\epsilon_{n-1}} \circ \cdots \circ s_{\epsilon_{n-p+1}}\right) .
\end{aligned}
$$

Set $m=n-p+1$, so that $t=m p$, and consider a rectangular tableau consisting of $p \times m$ boxes labeled from 1 to $m p$ as mentioned in the following figure. 


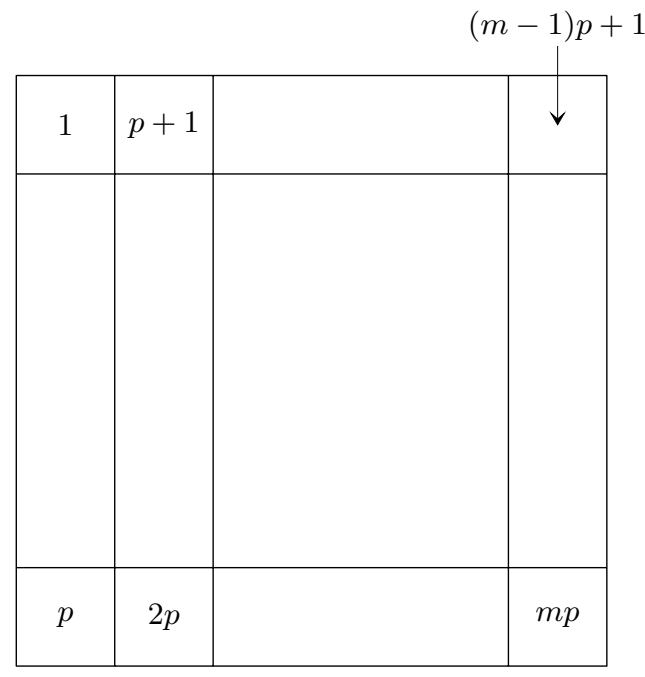

Following A. Postnikov [20, let us draw this tableau with $p$ wires going along the rows and $m$ wires going along the columns. Label the ends of the wires from 1 to $n+1$ as in the following figure.

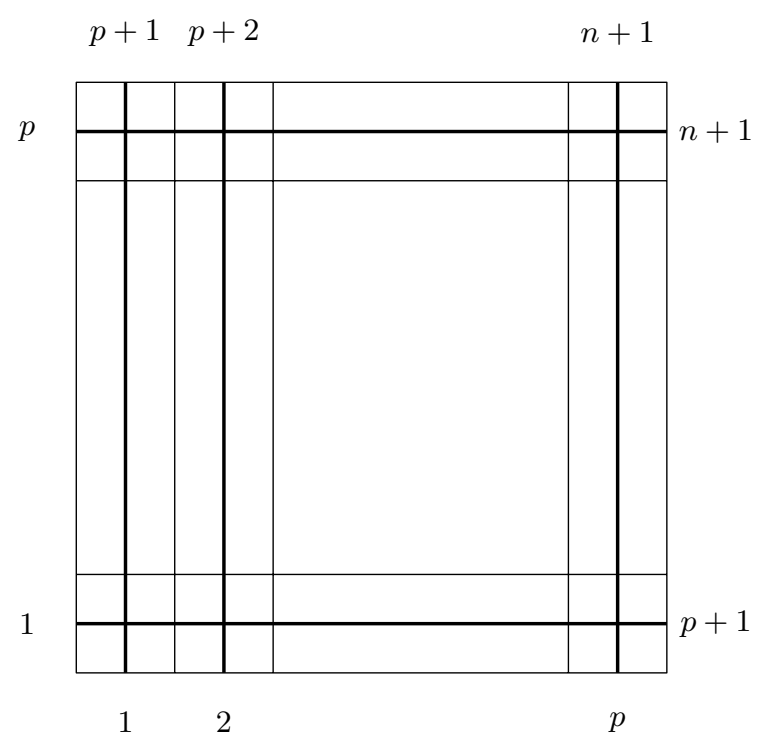

We observe that, if $W$ is identified as usual with the symmetric group $\mathcal{S}_{n+1}$ so that each $s_{\epsilon_{i}}$ is the transposition $(i, i+1)$, the wiring diagram defined above corresponds to the permutation $v=w^{-1}$. 
Now, consider any diagram $\Delta$ (i.e. any subset of $\llbracket 1, m p \rrbracket$ ), color in dark the boxes whose labels are in $\Delta$, and replace the corresponding crossing in noncolored boxes by a noncrossing:

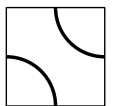

white box

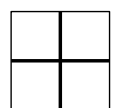

colored box

Then, if $\Delta=\left\{i_{1}<\cdots<i_{l}\right\}$, we observe that this new wiring diagram corresponds to the permutation $s_{\epsilon_{i_{l}}} \circ \cdots \circ s_{\epsilon_{i_{1}}}=v^{\Delta}$.

So, using A. Postnikov's conventions ([20], pp. 71 and 72) we can say:

(1) $\Delta$ is a pipe dream or a wiring diagram.

(2) $u=v^{\Delta}$ is the element of $W$ corresponding to the pipe dream $\Delta$ and $\Delta$ is a pipe dream (or a wiring diagram) of $u$.

In particular, we have:

(3) $v$ is the element of $W$ corresponding to the full pipe dream $\Delta=\llbracket 1, \ldots, t \rrbracket$.

(4) $I d$ is the element of $W$ corresponding to the empty pipe dream $\Delta=\emptyset$.

Now, in the general case, let us denote by $\leq$ the Bruhat order on the Weyl group $W$. Since (1) is a reduced expression of $w$, we have that (6) is a reduced expression of $v$. Let us recall ([13], corollary A.1.8) that an element $u$ of $W$ satisfies $u \leq w$ (resp. $u \leq v$ ) if and only if it can we written as a product of simple reflections obtained by omitting some of the $s_{\alpha_{i}}$ in the reduced expression (10) (resp. (6)). So, we have immediately:

Lemma 2.2.1. (1) The map $\Delta \mapsto u=w^{\Delta}$ (resp. $\left.u=v^{\Delta}\right)$ is surjective from the set of diagrams onto the set $\{u \in W \mid u \leq w\}$ (resp. $\{u \in W \mid u \leq v\}$ ).

(2) The map $u \mapsto u^{-1}$ is a bijection from $\{u \in W \mid u \leq w\}$ onto $\{u \in W \mid u \leq v\}$.

Lemma 2.2.2. $\boldsymbol{v}^{\Delta}:=\left(v_{0}^{\Delta}, v_{1}^{\Delta}, \ldots, v_{t}^{\Delta}\right)$ is a subexpression of (6) in the sense of Marsh and Rietsch 18 .

Proof. This is because $v_{i-1}^{\Delta}=s_{\alpha_{t}}^{\Delta} \ldots s_{\alpha_{t-i+2}}^{\Delta} \Longrightarrow v_{i}^{\Delta}=v_{i-1}^{\Delta} s_{\alpha_{t-i+1}}^{\Delta}$ with

$$
s_{\alpha_{t-i+1}}^{\Delta}=\left\{\begin{array}{cc}
s_{\alpha_{t-i+1}} & \text { if } t-i+1 \in \Delta \\
I d & \text { if } t-i+1 \notin \Delta
\end{array}\right\} .
$$

Lemma 2.2.3. If $\boldsymbol{u}=\left(u_{0}, u_{1}, \ldots, u_{t}\right)$ is a subexpression of $v$, there exists a unique diagram $\Delta$ such that $\boldsymbol{v}^{\Delta}=\boldsymbol{u}$. So, the map $\Delta \mapsto \boldsymbol{v}^{\Delta}$ is a bijection from the set of diagrams onto the set of subexpressions of $v$.

Proof. Since $\mathbf{u}$ is a subexpression of $v$, we have $u_{0}=I d$ and

$$
(\forall i \in \llbracket 1, \ldots, t \rrbracket)\left(u_{i-1}\right)^{-1} u_{i} \in\left\{s_{t-i+1}, I d\right\} .
$$

Given any diagram $\Delta$, we have $\mathbf{v}^{\Delta}=\left(v_{0}^{\Delta}, v_{1}^{\Delta}, \ldots, v_{t}^{\Delta}\right)$. Since $v_{0}^{\Delta}=u_{0}=I d$, we have $\mathbf{v}^{\Delta}=\mathbf{u}$ if and only if

$$
(\forall i \in \llbracket 1, \ldots, t \rrbracket)\left(u_{i-1}\right)^{-1} u_{i}=\left(v_{i-1}^{\Delta}\right)^{-1} v_{i}^{\Delta}=s_{\alpha_{t-i+1}}^{\Delta} .
$$

Now, there exists a unique diagram $\Delta$ which satisfies those conditions. It is defined by

$$
(\forall i \in \llbracket 1, \ldots, t \rrbracket)(t-i+1 \in \Delta) \Leftrightarrow\left(u_{i-1}\right)^{-1} u_{i}=s_{t-i+1} .
$$


Definition 2.2.2. We say that a diagram $\Delta$ is positive with respect to the reduced decomposition (11) (or that $\Delta$ is positive if there is no ambiguity) if $\mathbf{v}^{\Delta}=\left(v_{0}^{\Delta}, v_{1}^{\Delta}\right.$, $\left.\ldots, v_{t}^{\Delta}\right)$ is a positive subexpression of (6) in the sense of Marsh and Rietsch [18], namely:

$$
v_{i-1}^{\Delta}<v_{i-1}^{\Delta} s_{\alpha_{t-i+1}} \quad(\forall i \in \llbracket 1, \ldots, t \rrbracket) .
$$

Observation 2.2.1. The full diagram and the empty diagram are both positive.

Proof. • If $\Delta=\llbracket 1, \ldots, t \rrbracket$ is the full diagram, we have $v_{i-1}^{\Delta}=s_{\alpha_{t}} \ldots s_{\alpha_{t-i+2}}$ for any $i \in \llbracket 1, \ldots, t \rrbracket\left(v_{0}^{\Delta}=I d\right)$. Since (6) is a reduced expression of $v$, $s_{\alpha_{t}} \ldots s_{\alpha_{t-i+2}} s_{\alpha_{t-i+1}}$ is a reduced expression of $v_{i-1}^{\Delta} s_{\alpha_{t-i+1}}$. So, clearly, we have $v_{i-1}^{\Delta}<v_{i-1}^{\Delta} s_{\alpha_{t-i+1}}$.

- If $\Delta=\emptyset$, we have $v_{i-1}^{\Delta}=I d$, so that $v_{i-1}^{\Delta}<v_{i-1}^{\Delta} s_{\alpha_{t-i+1}}$.

Consider any $i \in \llbracket 1, \ldots, t \rrbracket$, set $j=t-i+1$ and $\left\{j_{1}<\cdots<j_{s}\right\}=\Delta \cap \llbracket j+$ $1, \ldots, t \rrbracket=\Delta \cap \llbracket t-i+2, \ldots, t \rrbracket$, so that $v_{i-1}^{\Delta}=s_{\alpha_{t}}^{\Delta} \ldots s_{\alpha_{t-i+2}}^{\Delta}=s_{\alpha_{j_{s}}}^{\Delta} \ldots s_{\alpha_{j_{1}}}$ and $v_{i-1}^{\Delta} s_{\alpha_{t-i+1}}=s_{\alpha_{j_{s}}} \ldots s_{\alpha_{j_{1}}} s_{\alpha_{j}}$.

If $w_{1}$ and $w_{2}$ are in $W$, it follows from ([13], corollary A.1.8) that

$\left(w_{1}<w_{2}\right) \Leftrightarrow\left(w_{1} \neq w_{2}\right.$ and $w_{1}$ is obtained by omitting some simple reflections in a reduced expression of $w_{2}$ )

$$
\Leftrightarrow\left(w_{1}^{-1}<w_{2}^{-1}\right) \text {. }
$$

So, with $w_{1}=v_{i-1}^{\Delta}$ and $w_{2}=v_{i-1}^{\Delta} s_{\alpha_{t-i+1}}$, we obtain:

$$
\left(v_{i-1}^{\Delta}<v_{i-1}^{\Delta} s_{\alpha_{t-i+1}}\right) \Leftrightarrow\left(s_{\alpha_{j_{1}}} \ldots s_{\alpha_{j_{s}}}<s_{\alpha_{j}} s_{\alpha_{j_{1}}} \ldots s_{\alpha_{j_{s}}}\right) .
$$

Let us set $u=s_{\alpha_{j_{1}}} \ldots s_{\alpha_{j_{s}}}$ and recall that $l\left(s_{\alpha_{j}} u\right)=l(u) \pm 1$. If $l\left(s_{\alpha_{j}} u\right)=l(u)$ +1 , we have that $u<s_{\alpha_{j}} u$ ([13], A.1.6). Conversely, if $u<s_{\alpha_{j}} u$, we have $l(u)$ $<l\left(s_{\alpha_{j}} u\right)\left(\left[13\right.\right.$, A.1.6) and, consequently, $l\left(s_{\alpha_{j}} u\right)=l(u)+1$. So, we have:

$$
\left(v_{i-1}^{\Delta}<v_{i-1}^{\Delta} s_{\alpha_{t-i+1}}\right) \Leftrightarrow l\left(s_{\alpha_{j}} s_{\alpha_{j_{1}}} \ldots s_{\alpha_{j_{s}}}\right)=1+l\left(s_{\alpha_{j_{1}}} \ldots s_{\alpha_{j_{s}}}\right) .
$$

Since the mapping $i \mapsto j=t-i+1$ is a bijection of $\llbracket 1, \ldots, t \rrbracket$, we conclude:

Lemma 2.2.4. The diagram $\Delta$ is positive if and only if, for any $j \in \llbracket 1, \ldots, t \rrbracket$,

$$
\Delta \cap \llbracket j+1, \ldots, t \rrbracket=\left\{j_{1}<\cdots<j_{s}\right\} \Rightarrow l\left(s_{\alpha_{j}} s_{\alpha_{j_{1}}} \ldots s_{\alpha_{j_{s}}}\right)=1+l\left(s_{\alpha_{j_{1}}} \ldots s_{\alpha_{j_{s}}}\right) .
$$

Now, we can prove the following characterization of positive diagrams.

Proposition 2.2.1. The diagram $\Delta$ is positive if and only if, for any $j \in \llbracket 1, \ldots, t \rrbracket$,

$$
\Delta \cap \llbracket j+1, \ldots, t \rrbracket=\left\{j_{1}<\cdots<j_{s}\right\} \Rightarrow l\left(s_{\alpha_{j}} s_{\alpha_{j_{1}}} \ldots s_{\alpha_{j_{s}}}\right)=1+s .
$$

Proof. Assume that $\Delta$ is positive, choose $j, j_{1}, \ldots, j_{s}$ as above, and consider any $l$ with $1<l<s$. We have $\Delta \cap \llbracket j_{l}+1, \ldots, t \rrbracket=\left\{j_{l+1}<\cdots<j_{s}\right\}$ and, by lemma 2.2.4, $l\left(s_{\alpha_{j_{l}}} s_{\alpha_{j_{l+1}}} \ldots s_{\alpha_{j_{s}}}\right)=1+l\left(s_{\alpha_{j_{l+1}}} \ldots s_{\alpha_{j_{s}}}\right)$. From this, we get that $l\left(s_{\alpha_{j_{1}}} \ldots s_{\alpha_{j_{s}}}\right)$ $=s$ and, again by lemma $2.2 .4, l\left(s_{\alpha_{j}} s_{\alpha_{j_{1}}} \ldots s_{\alpha_{j_{s}}}\right)=1+s$.

Conversely, assume that this equality holds for any $j$ and choose $j, j_{1}, \ldots, j_{s}$ as above. We have $\Delta \cap \llbracket j_{1}+1, \ldots, t \rrbracket=\left\{j_{2}<\cdots<j_{s}\right\}$ and, by assumption, $l\left(s_{\alpha_{j_{1}}} s_{\alpha_{j_{2}}} \ldots s_{\alpha_{j_{s}}}\right)=1+(s-1)=s$. So, $l\left(s_{\alpha_{j}} s_{\alpha_{j_{1}}} \ldots s_{\alpha_{j_{s}}}\right)=1+l\left(s_{\alpha_{j_{1}}} \ldots s_{\alpha_{j_{s}}}\right)$ and $\Delta$ is positive by lemma 2.2.4. 
2.3. Some properties of positive diagrams. In this section, we use the same conventions as in section 2.2 .

\section{Proposition 2.3.1.}

(1) The map: $\Delta \mapsto u=v^{\Delta}$ is a bijection from the set of positive diagrams onto the set $\{u \in W \mid u \leq v\}$.

(2) The map: $\Delta \mapsto u=w^{\Delta}$ is a bijection from the set of positive diagrams onto the set $\{u \in W \mid u \leq w\}$.

Proof. (1) By lemma 2.2.1, $f: \Delta \mapsto u=v^{\Delta}$ is a map from the set of positive diagrams in the set $\{u \in W \mid u \leq v\}$. Consider any $u \in W$ with $u \leq v$. By ([18, Lemma 3.5.), there exists a unique positive subexpression $\mathbf{u}^{+}=\left(u_{0}, u_{1}, \ldots, u_{t}\right)$ of (6) with $u_{t}=u$. By lemma 2.2. 3, there exists a unique diagram $\Delta$ which satisfies $\mathbf{v}^{\Delta}=\left(v_{0}^{\Delta}, v_{1}^{\Delta}, \ldots, v_{t}^{\Delta}\right)=\mathbf{u}^{+}$. So, $\Delta$ is positive (def. 2.2.2) and $v^{\Delta}=v_{t}^{\Delta}=u_{t}=u$. This proves that $f$ is surjective.

If $\Delta^{\prime}$ is a positive diagram such that $v^{\Delta^{\prime}}=u$, then $\mathbf{v}^{\Delta^{\prime}}=\left(v_{0}^{\Delta^{\prime}}, v_{1}^{\Delta^{\prime}}\right.$, $\ldots, v_{t}^{\Delta^{\prime}}$ ) is a positive subexpression of (6) with $v_{t}^{\Delta^{\prime}}=v^{\Delta^{\prime}}=u$. So, we have $\mathbf{v}^{\Delta^{\prime}}=\mathbf{u}^{+} \Rightarrow \mathbf{v}^{\Delta^{\prime}}=\mathbf{v}^{\Delta}$ and, by lemma 2.2.3, $\Delta^{\prime}=\Delta$. This proves that $f$ is bijective.

(2) Denote by $g$ the bijection $u \mapsto u^{-1}$ from $\{u \in W \mid u \leq w\}$ onto $\{u \in W \mid u \leq$ $v\}$ (lemma 2.2. 1).

Then $g^{-1} \circ f: \Delta \mapsto u=\left(v^{\Delta}\right)^{-1}=w^{\Delta}$ is a bijection from the set of positive diagrams onto the set $\{u \in W \mid u \leq w\}$.

Consider any $p \in \llbracket 1, \ldots, t \rrbracket, p \neq 1, t$, and set

$$
\begin{gathered}
w_{1}=s_{\alpha_{1}} \ldots s_{\alpha_{p}}, \\
w_{2}=s_{\alpha_{p+1}} \ldots s_{\alpha_{t}} .
\end{gathered}
$$

We have $w=w_{1} w_{2}$ and, since (11) is a reduced expression of $w$, (17) and (8) are reduced expressions of $w_{1}$ and $w_{2}$, respectively.

Denote by $\Delta$ any subset of $\llbracket 1, \ldots, p \rrbracket$, so that $\Delta$ is a diagram with respect to (77) and with respect to (1) both.

Proposition 2.3.2. ( $\Delta$ is positive with respect to (7) $) \Leftrightarrow(\Delta$ is positive with respect to (10).

Proof. Assume that $\Delta$ is positive with respect to (11) and consider any $j \in \llbracket 1, \ldots$, $p \rrbracket$. We have $\Delta \cap \llbracket j+1, \ldots, p \rrbracket=\Delta \cap \llbracket j+1, \ldots, t \rrbracket$ and, using the characterization of positive diagrams given in proposition 2.2 .1 , we obtain that $\Delta$ is positive with respect to (7).

Assume that $\Delta$ is positive with respect to (17) and consider any $j \in \llbracket 1, \ldots, t \rrbracket$. If $j \leq p$, we have $\Delta \cap \llbracket j+1, \ldots, t \rrbracket=\Delta \cap \llbracket j+1, \ldots, p \rrbracket$, so that the characterization given in proposition 2.2.1 is satisfied. If $j>p$, we have $\Delta \cap \llbracket j+1, \ldots, t \rrbracket=\emptyset$, so that the characterization given in proposition 2.2.1 is again satisfied.

Now, consider a nonempty diagram $\Delta$ (with respect to (1)) and two integers $j$, $m$ in $\llbracket 1, \ldots, t \rrbracket$ with $j<m$ and $m \in \Delta$. Let us recall that $\left(\beta_{1}, \ldots, \beta_{t}\right)$ is the sequence of positive roots associated to the reduced expression (1D) of $w$ (section 2.1) and let us denote:

$$
\text { - } \bar{\Delta}=\llbracket 1, \ldots, t \rrbracket \backslash \Delta,
$$


- $\Delta \cap \llbracket j+1, \ldots, m-1 \rrbracket=\left\{j_{1}<\cdots<j_{r}\right\}$ (unless this set is empty),

- $\bar{\Delta} \cap \llbracket j+1, \ldots, m-1 \rrbracket=\left\{l_{1}<\cdots<l_{p}\right\}$ (unless this set is empty),

- $\left(\gamma_{1}, \ldots, \gamma_{p}, \gamma_{p+1}\right)$ is the sequence of (not necessarily positive) roots defined recursively by $\gamma_{p+1}=\beta_{m}$ and, for $1 \leq i \leq p, \gamma_{i}=s_{\beta_{l_{i}}}\left(\gamma_{i+1}\right)$,

- for $1 \leq i \leq p$, we set $a_{i}=\left(\beta_{l_{i}}^{\vee}, \gamma_{i+1}\right)$.

Until the end of this section, we assume that $\bar{\Delta} \cap \llbracket j+1, \ldots, m-1 \rrbracket$ is nonempty, so that $p$ and the roots $\gamma_{i}(1 \leq i \leq p+1)$ are well defined.

Lemma 2.3.1.

$$
\gamma_{1}=\beta_{m}-a_{p} \beta_{l_{p}}-\cdots-a_{1} \beta_{l_{1}} .
$$

Proof. For $1 \leq i \leq p$, we have $\gamma_{i}=\gamma_{i+1}-a_{i} \beta_{l_{i}}$. Summing these equalities, we get $\gamma_{1}=\gamma_{p+1}-a_{p} \beta_{l_{p}}-\cdots-a_{1} \beta_{l_{1}}$ and, by definition, $\gamma_{p+1}=\beta_{m}$.

Set

$$
w^{\prime}=s_{\alpha_{1}} \ldots s_{\alpha_{m-1}}
$$

and observe that, since (11) is a reduced expression of $w$, (9) is a reduced expression of $w^{\prime}$.

Lemma 2.3.2. For any $i \in \llbracket 1, \ldots, p \rrbracket$, denote by $w_{i}^{\prime}$ the element of $W$ obtained by omitting $s_{\alpha_{l_{i}}}, \ldots, s_{\alpha_{l_{p}}}$ in (9). Then, we have $\gamma_{i}=w_{i}^{\prime}\left(\alpha_{m}\right)$.

Proof. Assume that $i=p$ and set $u_{p}=s_{\alpha_{1}} \ldots s_{\alpha_{l_{p}}-1}$, so that:

$$
\begin{aligned}
w^{\prime}=u_{p} s_{\alpha_{l_{p}}} s_{\alpha_{l_{p}+1}} \ldots s_{\alpha_{m-1}} & \Rightarrow u_{p}^{-1} w^{\prime}=s_{\alpha_{l_{p}}} s_{\alpha_{l_{p}+1}} \ldots s_{\alpha_{m-1}} \\
& \Rightarrow u_{p} s_{\alpha_{l_{p}}} u_{p}^{-1} w^{\prime}=u_{p} s_{\alpha_{l_{p}+1}} \ldots s_{\alpha_{m-1}}=w_{p}^{\prime} .
\end{aligned}
$$

As $u_{p}\left(\alpha_{l_{p}}\right)=\beta_{l_{p}}$, we conclude that $w_{p}^{\prime}=s_{\beta_{l_{p}}} w^{\prime}$. So, $w_{p}^{\prime}\left(\alpha_{m}\right)=s_{\beta_{l_{p}}} w^{\prime}\left(\alpha_{m}\right)=$ $s_{\beta_{l_{p}}}\left(\beta_{m}\right)=\gamma_{p}$.

Assume $i<p, \gamma_{i+1}=w_{i+1}^{\prime}\left(\alpha_{m}\right)$, and set $u_{i}=s_{\alpha_{1}} \ldots s_{\alpha_{l_{i}}-1}$. We have $w_{i+1}^{\prime}=$ $u_{i} s_{\alpha_{l_{i}}} \sigma$ with $\sigma \in W$ and $w_{i}^{\prime}=u_{i} \sigma$. From this, we deduce (as in the case $i=p$ ) that $u_{i} s_{\alpha_{l_{i}}} u_{i}^{-1} w_{i+1}^{\prime}=w_{i}^{\prime}$. So, $w_{i}^{\prime}\left(\alpha_{m}\right)=s_{\beta_{l_{i}}} w_{i+1}^{\prime}\left(\alpha_{m}\right)=s_{\beta_{l_{i}}}\left(\gamma_{i+1}\right)=\gamma_{i}$.

Now, we can prove:

Proposition 2.3.3. Assume that

$$
\beta_{j}+\beta_{m}=a_{p} \beta_{l_{p}}+\cdots+a_{1} \beta_{l_{1}} .
$$

Then $\Delta$ is not positive (with respect to (10).

Proof. By lemma 2.3.1, we have $\beta_{j}=-\gamma_{1}$ and, by lemma 2.3.2, we can write $\beta_{j}$ $=-w_{1}^{\prime}\left(\alpha_{m}\right)$. Recall that $w_{1}^{\prime}$ is obtained by omitting $s_{\alpha_{l_{1}}}, \ldots, s_{\alpha_{l_{p}}}$ in the reduced expression (9) of $w^{\prime}$ and that

$$
\begin{aligned}
\left\{l_{1}<\cdots<l_{p}\right\} & =\bar{\Delta} \cap \llbracket j+1, \ldots, m-1 \rrbracket \\
& =\llbracket j+1, \ldots, m-1 \rrbracket \backslash\left\{j_{1}<\cdots<j_{r}\right\} .
\end{aligned}
$$

- In the case $\Delta \cap \llbracket j+1, \ldots, m-1 \rrbracket=\emptyset$, we have $\left\{l_{1}, \ldots, l_{p}\right\}=\llbracket j+1, m-1 \rrbracket$, so that $w_{1}^{\prime}=s_{\alpha_{1}} \ldots s_{\alpha_{j-1}} s_{\alpha_{j}}$. So, we have $\beta_{j}=s_{\alpha_{1}} \ldots s_{\alpha_{j-1}}\left(\alpha_{j}\right)=$ $-s_{\alpha_{1}} \ldots s_{\alpha_{j-1}} s_{\alpha_{j}}\left(\alpha_{m}\right)$. This implies that $\alpha_{j}=-s_{\alpha_{j}}\left(\alpha_{m}\right)$, so $\alpha_{m}=\alpha_{j}$. Now, we have $\Delta \cap \llbracket j+1, \ldots, t \rrbracket=\left\{m=j_{1}^{\prime}<\cdots<j_{l}^{\prime}\right\}(l \geq 1)$.

Since $\alpha_{j}=\alpha_{m}=\alpha_{j_{1}^{\prime}}, l\left(s_{\alpha_{j}} s_{\alpha_{j_{1}^{\prime}}} \ldots s_{\alpha_{j_{l}^{\prime}}}\right) \leq l-1$, proposition 2.2.1 implies that $\Delta$ is not positive. 
- Otherwise, we have

$$
w_{1}^{\prime}=s_{\alpha_{1}} \ldots s_{\alpha_{j-1}} s_{\alpha_{j}} s_{\alpha_{j_{1}}} \ldots s_{\alpha_{j_{r}}}=\eta s_{\alpha_{j}} s_{\alpha_{j_{1}}} \ldots s_{\alpha_{j_{r}}}
$$

with $\eta=s_{\alpha_{1}} \ldots s_{\alpha_{j-1}}$.

Since $\beta_{j}=\eta\left(\alpha_{j}\right)$, we have $\beta_{j}=-w_{1}^{\prime}\left(\alpha_{m}\right) \Rightarrow \alpha_{j}=-s_{\alpha_{j}} s_{\alpha_{j_{1}}} \ldots s_{\alpha_{j_{r}}}\left(\alpha_{m}\right)$ $\Rightarrow s_{\alpha_{j}} s_{\alpha_{j_{1}}} \ldots s_{\alpha_{j_{r}}}\left(\alpha_{m}\right)=-\alpha_{j}$ is a negative root. By ([10], lemma 10.2.C), this implies that $l\left(s_{\alpha_{j}} s_{\alpha_{j_{1}}} \ldots s_{\alpha_{j_{r}}} s_{\alpha_{m}}\right)<r+2$.

As $m \in \Delta$, we have $\Delta \cap \llbracket j+1, \ldots, t \rrbracket=\left\{j_{1}<\cdots<j_{s}\right\}$ with $s>r$ and $j_{r+1}=m$. So, $l\left(s_{\alpha_{j}} s_{\alpha_{j_{1}}} \ldots s_{\alpha_{j_{s}}}\right) \leq l\left(s_{\alpha_{j}} s_{\alpha_{j_{1}}} \ldots s_{\alpha_{j_{r}}} s_{\alpha_{m}}\right)+s-(r+1)$ $<r+2+s-(r+1)=s+1$ and, by proposition 2.2.1, $\Delta$ is not positive.

\section{BACKGROUND ON THE DELETING DERIVATIONS ALGORITHM}

3.1. Conventions. In this section, we use the conventions of section 2.1 and we set $R=U^{+}[w]$. In order to simplify the notation, we set

- $X_{i}=X_{\beta_{i}}$ for any $i \in \llbracket 1, \ldots, t \rrbracket$, so that $R=k\left\langle X_{1}, \ldots, X_{t}\right\rangle$. Moreover, $X_{1}, \ldots, X_{t}$ are called the canonical generators (with respect to the reduced decomposition (10) of $R$.

- Recall (section 2.1) that, for each $\rho$ in the root lattice $\mathbb{Z} \Pi$, the map $h_{\rho}: u$ $\mapsto K_{\rho} . u$ is in $A u t(R)$, the group of automorphisms of the algebra $R$.

- Let us set $H=\left\{h_{\rho} \mid \rho \in \mathbb{Z} \Pi\right\}$ and observe that $H$ is an abelian subgroup of Aut $(R)$.

- Recall that $R$ is $\mathbb{Z} \Pi$-graded and that, for any homogeneous element $u$ of degree $\gamma$ in $R$, for any $\rho \in \mathbb{Z} \Pi$, we have $h_{\rho}(u)=q^{-(\rho, \gamma)} u$.

- For each $(i, j) \in(\llbracket 1, \ldots, t \rrbracket)^{2}$, set $\lambda_{i, j}=q^{-\left(\beta_{i}, \beta_{j}\right)}, q_{i}=\lambda_{i, i}=q^{-\left\|\beta_{i}\right\|^{2}}$, and observe that $q_{i}$ is not a root of unity.

- If $1 \leq i<j \leq t$, the Levendorskii-Soibelman formula can be written as

$$
P_{j, i}=\sum_{\underline{a}=\left(a_{i+1}, \ldots, a_{j-1}\right)} c_{\underline{a}} X_{i+1}^{a_{i+1}} \ldots X_{j-1}^{a_{j-1}}
$$

with $\underline{a} \in \mathbb{N}^{j-i-1}, c_{\underline{a}} \in k$, and $c_{\underline{a}} \neq 0$ for only finitely many $\underline{a}$. Moreover, $P_{j, i}$ is homogeneous with degree $\beta_{i}+\beta_{j}$ so that, if $j=i+1$, we have $P_{j, i}=0$. In the general case, this also implies that, if $c_{a} \neq 0$, then $a_{i+1} \beta_{i+1}+\cdots+$ $a_{j-1} \beta_{j-1}=\beta_{i}+\beta_{j}$.

- Since $\beta_{i}$ and $\beta_{j}$ are positive roots, $\beta_{i}+\beta_{j}$ is nonzero. So, if $c_{\underline{a}} \neq 0$, then $\underline{a}$ is nonzero.

- From this, we get that the generators $X_{1}, \ldots, X_{t}$ of $R$ satisfy the equalities and the assumption 6.1.1. of (4], section 6.1.).

- Moreover, since the ordered monomials $X^{\underline{a}}:=X_{1}^{a_{1}} \ldots X_{t}^{a_{t}}, \underline{a}=\left(a_{1}, \ldots, a_{1}\right)$ $\in \mathbb{N}^{t}$, are a basis of $R$, it follows from ([4, propositions 6.1.1. and 6.1.2.) that $R$ satisfies the conventions of (4], section 3.1.). In particular, $R$ is an iterated Ore extension of the ground field $k$ and so, $R$ is a noetherian domain.

- We denote by $F=\operatorname{Fract}(R)$ the division ring of fractions of $R$.

- By (4, proposition 6.1.1.), we also get that $R$ is the $k$-algebra generated by the "variables" $X_{1}, \ldots, X_{t}$ subject to the relations (10). 
- For each $l \in \llbracket 1, \ldots, t \rrbracket$, we set $h_{l}=h_{\beta_{l}} \in H$, and observe that, if $i \in \llbracket 1$, $\ldots, t \rrbracket$, we have $h_{l}\left(X_{i}\right)=\lambda_{l, i} X_{i}$.

- Since each $X_{i}$ is homogeneous, it is an $H$-eigenvector and, since $\lambda_{1,1}=$ $q^{-\left(\beta_{1}, \beta_{1}\right)}$ is not a root of unity, the assumption 4.1.2. of [4] is satisfied. Since each $\lambda_{i, j}$ is a power of $q$, the assumption 4.1.1. of [4] is also satisfied. As explained in [4] (proof of lemma 4.2.2.) this implies that each prime ideal of $R$ is completely prime.

- Recall that each automorphism $h \in H$ can be extended to a (unique) automorphism (denoted $h$ also) of $F$, so that $H$ can be seen as a subgroup of $\operatorname{Aut}(F)$.

3.2. The algebras $R^{(m)}$. Recall (4, section 3) that, for any $m \in \llbracket 2, \ldots, t+1 \rrbracket$, there exists a family $\left(X_{1}^{(m)}, \ldots, X_{t}^{(m)}\right)$ of new "variables" in $F$, called the canonical generators (with respect to the reduced decomposition (1)) of the algebra $R^{(m)}=$ $k\left\langle X_{1}^{(m)}, \ldots, X_{t}^{(m)}\right\rangle$, and which satisfies the following properties:

- If $1 \leq i<j \leq t$, we have the following simplified Levendorskii-Soibelman formula:

$$
\begin{array}{ll} 
& X_{j}^{(m)} X_{i}^{(m)}-\lambda_{j, i} X_{i}^{(m)} X_{j}^{(m)}=P_{j, i}^{(m)} \text { with } \\
\diamond \quad & m \leq j \Rightarrow P_{j, i}^{(m)}=0, \\
\diamond \quad & j<m \Rightarrow P_{j, i}^{(m)}=\sum_{\underline{a}=\left(a_{i+1}, \ldots, a_{j-1}\right)} c_{\underline{a}}\left(X_{i+1}^{(m)}\right)^{a_{i+1}} \ldots\left(X_{j-1}^{(m)}\right)^{a_{j-1},}
\end{array}
$$

where the coefficients $c_{\underline{a}}$ are the same as in (11). $\left(S o, P_{j, i}^{(m)}=0\right.$ in the case $i+1=j<m$ and, in the general case, if $c_{\underline{a}} \neq 0$, we have $a_{i+1} \beta_{i+1}+\cdots$ $+a_{j-1} \beta_{j-1}=\beta_{i}+\beta_{i}$, so that $\underline{a}$ is nonzero.)

- This implies that the generators $X_{1}^{(m)}, \ldots, X_{t}^{(m)}$ of $R^{(m)}$ still satisfy the equalities and the assumption 6.1.1. of (4, section 6.1.).

- The ordered monomials $\left(X^{(m)}\right)^{\underline{a}}:=\left(X_{1}^{(m)}\right)^{a_{1}} \ldots\left(X_{t}^{(m)}\right)^{a_{t}}, \underline{a}=\left(a_{1}, \ldots, a_{1}\right)$ $\in \mathbb{N}^{t}$, are a basis of $R^{(m)}$. So, $R^{(m)}$ still satisfies the conventions of (4, section 3.1.). In particular, $R^{(m)}$ is an iterated Ore extension of the ground field $k$ and so, $R^{(m)}$ is a noetherian domain.

- We have $\operatorname{Fract}\left(R^{(m)}\right)=\operatorname{Fract}(R)=F$.

- By (4], proposition 6.1.1.), $R^{(m)}$ is the $k$-algebra generated by the "variables" $X_{1}^{(m)}, \ldots, X_{t}^{(m)}$ subject to the relations (12).

- For each $\rho$ in the root lattice $\mathbb{Z} \Pi$ and for each $i \in \llbracket 1, \ldots, t \rrbracket$, we still have $h_{\rho}\left(X_{i}^{(m)}\right)=q^{-\left(\rho, \beta_{i}\right)} X_{i}^{(m)}$. This implies that $h_{\rho}\left(R^{(m)}\right)=R^{(m)}$. So $H$ can still be seen as a subgroup of $A u t\left(R^{(m)}\right)$ and each $X_{i}^{(m)}$ is an $H$-eigenvector. If $l$ and $i$ are in $\llbracket 1, \ldots, t \rrbracket$, we have $h_{l}\left(X_{i}^{(m)}\right)=\lambda_{l, i} X_{i}^{(m)}$ and, as above, this implies that $R^{(m)}$ satisfies the assumptions 4.4.2 and 4.4.1 of [4. As explained in (4), proof of lemma 4.2.2), this implies that each prime ideal of $R^{(m)}$ is completely prime.

- If $u \in F$, and if $\gamma \in \mathbb{Z} \Pi$, we say that $u$ is homogeneous of degree $\gamma$, if $h_{\rho}(u)$ $=q^{-(\rho, \gamma)} u$ for all $\rho$ in $\mathbb{Z} \Pi$. So, for example, each $X_{i}^{(m)}$ is homogeneous of degree $\beta_{i}$.

- If $u_{1}, \ldots, u_{r}$ are homogeneous of same degree $\gamma$, then any linear combination of $u_{1}, \ldots, u_{r}$ (with coefficients in $k$ ) is homogeneous of degree $\gamma$. 
- Clearly, if $u_{1}$ is homogeneous of degree $\gamma_{1}$ and $u_{2}$ is homogeneous of degree $\gamma_{2}$, then $u_{1} u_{2}$ is homogeneous of degree $\gamma_{1}+\gamma_{2}$.

- Likewise, if $u$ is nonzero and homogeneous of degree $\gamma$, then $u^{-1}$ is homogeneous of degree $-\gamma$.

- So, if $u_{1}, \ldots, u_{r}$ are nonzero and homogeneous of degrees $\gamma_{1}, \ldots, \gamma_{r}$, respectively, if $\underline{a}=\left(a_{1}, \ldots, a_{r}\right) \in \mathbb{Z}^{r}$, then $u^{\underline{a}}:=u_{1}^{a_{1}} \ldots u_{r}^{a_{r}}$ is homogeneous of degree $a_{1} \gamma_{1}+\cdots+a_{r} \gamma_{r}$.

- For each $j \in \llbracket 1, \ldots, t \rrbracket$, denote by $\delta_{j}^{(m)}$ the left $h_{j}$-derivation of $R^{(m)}$ defined by $X_{j}^{(m)}$. This means that, for each $a \in R^{(m)}, \delta_{j}^{(m)}(a):=X_{j}^{(m)} a-$ $h_{j}(a) X_{j}^{(m)}$.

- If $j \in \llbracket 1, \ldots, t \rrbracket$, we have

$$
\begin{gathered}
h_{j}\left(k\left\langle X_{1}^{(m)}, \ldots, X_{j-1}^{(m)}\right\rangle\right)=k\left\langle X_{1}^{(m)}, \ldots, X_{j-1}^{(m)}\right\rangle, \\
\delta_{j}^{(m)}\left(k\left\langle X_{1}^{(m)}, \ldots, X_{j-1}^{(m)}\right\rangle\right) \subset k\left\langle X_{1}^{(m)}, \ldots, X_{j-1}^{(m)}\right\rangle .
\end{gathered}
$$

So, $h_{j}$ induces an automorphism, still denoted $h_{j}$, of the algebra $k\left\langle X_{1}^{(m)}, \ldots, X_{j-1}^{(m)}\right\rangle$. It is the only automorphism of $k\left\langle X_{1}^{(m)}, \ldots, X_{j-1}^{(m)}\right\rangle$ which satisfies $h_{j}\left(X_{i}^{(m)}\right)=$ $\lambda_{j, i} X_{i}^{(m)}$ for any $i$.

Likewise, $\delta_{j}^{(m)}$ induces a left $h_{j}$-derivation of $k\left\langle X_{1}^{(m)}, \ldots, X_{j-1}^{(m)}\right\rangle$, still denoted $\delta_{j}^{(m)}$. It satisfies the following properties:

(1) If $1 \leq i<j$, we have $\delta_{j}^{(m)}\left(X_{i}^{(m)}\right)=P_{j, i}^{(m)}$. This implies that $\delta_{j}^{(m)}$ is zero on $k\left\langle X_{1}^{(m)}, \ldots, X_{j-1}^{(m)}\right\rangle$, and that $X_{j}^{(m)} a=h_{j}(a) X_{j}^{(m)}$ for any $a$ in $k\left\langle X_{1}^{(m)}, \ldots, X_{j-1}^{(m)}\right\rangle$, as soon as $j \geq m$.

(2) $\delta_{j}^{(m)}$ is locally nilpotent on $k\left\langle X_{1}^{(m)}, \ldots, X_{j-1}^{(m)}\right\rangle$.

(3) We have

$$
R^{(m)}=k\left[X_{1}^{(m)}\right]\left[X_{2}^{(m)} ; h_{2}, \delta_{2}^{(m)}\right] \ldots\left[X_{m-1}^{(m)} ; h_{m-1}, \delta_{m-1}^{(m)}\right]\left[X_{m}^{(m)} ; h_{m}\right] \ldots\left[X_{t}^{(m)} ; h_{t}\right] .
$$

- For each $i \in \llbracket 1, \ldots, t \rrbracket$, we have $X_{i}^{(t+1)}=X_{i}$, so that $R^{(t+1)}=R$.

Until the end of this section, we assume that $m \in \llbracket 2, \ldots, t \rrbracket$.

- Recall that $q_{m}=\lambda_{m, m}$ is not a root of unity and define quantum integers $[l]_{q_{m}}$ (resp. quantum factorial $\left.[l] !_{q_{m}}\right)$ for $l \in \mathbb{N}$, as in (4], section 2).

- For each $i \in \llbracket 1, \ldots, t \rrbracket$, we have

(1) $m \leq i \Rightarrow X_{i}^{(m)}=X_{i}^{(m+1)}$. In particular, we have $X_{m}^{(m)}=X_{m}^{(m+1)}$.

(2) $i<m \Rightarrow X_{i}^{(m)}=X_{i}^{(m+1)}+\sum_{l=1}^{+\infty} C_{l}^{(m+1)}\left(X_{m}^{(m+1)}\right)^{-l}$ with

$$
C_{l}^{(m+1)}=\frac{\left(1-q_{m}\right)^{-l}}{[l] !_{q_{m}}} \lambda_{m, i}^{-l}\left(\delta_{m}^{(m+1)}\right)^{l}\left(X_{i}^{(m+1)}\right) .
$$

- We observe that, since $k\left\langle X_{1}^{(m+1)}, \ldots, X_{m-1}^{(m+1)}\right\rangle$ is $\delta_{m}^{(m+1)}$-stable, we have $C_{l}^{(m+1)} \in k\left\langle X_{1}^{(m+1)}, \ldots, X_{m-1}^{(m+1)}\right\rangle$ and, since $\delta_{m}^{(m+1)}$ is locally nilpotent on $k\left\langle X_{1}^{(m+1)}, \ldots, X_{m-1}^{(m+1)}\right\rangle$, only finitely many $C_{l}^{(m+1)}$ are nonzero.

- If $i<m$ and $X_{m}^{(m+1)} X_{i}^{(m+1)}-\lambda_{m, i} X_{i}^{(m+1)} X_{m}^{(m+1)}=P_{m, i}^{(m+1)}=0$, then $\delta_{m}^{(m+1)}\left(X_{i}^{(m+1)}\right)=0$, and $X_{i}^{(m)}=X_{i}^{(m+1)}$. 
- There exists a unique homomorphism of $k$-algebras

$$
\Theta^{(m)}: k\left\langle X_{1}^{(m+1)}, \ldots, X_{m-1}^{(m+1)}\right\rangle \rightarrow k\left\langle X_{1}^{(m)}, \ldots, X_{m-1}^{(m)}\right\rangle
$$

which satisfies $\Theta^{(m)}\left(X_{i}^{(m+1)}\right)=X_{i}^{(m)}$ for $1 \leq i \leq m-1$.

- When $m=2$, we set

(1) $Z_{i}=X_{i}^{(2)}$ for $i \in \llbracket 1, \ldots, t \rrbracket$.

(2) $\bar{R}=R^{(2)}$.

- $\bar{R}=k\left\langle Z_{1}, \ldots, Z_{t}\right\rangle$ is the $k$-algebra generated by the variables $Z_{1}, \ldots, Z_{t}$ subject to the relations:

$$
(1 \leq i<j \leq t) \Rightarrow Z_{j} Z_{i}=\lambda_{j, i} Z_{i} Z_{j}
$$

- For any $i \in \llbracket 1, \ldots, t \rrbracket$, we have $X_{i}^{(2)}=\cdots=X_{i}^{(i+1)}=Z_{i}$.

- $S_{m}=\left\{\left(X_{m}^{(m+1)}\right)^{l} \mid l \in \mathbb{N}\right\}=\left\{\left(X_{m}^{(m)}\right)^{l} \mid l \in \mathbb{N}\right\}$ is a multiplicative system of regular elements in $R^{(m)}$ and in $R^{(m+1)}$. It satisfies the Ore condition (on both sides) in each one of those rings and we have $R^{(m)} S_{m}^{-1}=R^{(m+1)} S_{m}^{-1}$.

3.3. Prime and $H$-prime spectrum of $R^{(m)}$, admissible diagrams. Consider any integer $m \in \llbracket 2, \ldots, t+1 \rrbracket$. As usual, we denote by $\operatorname{Spec}\left(R^{(m)}\right)$ (resp. $H-$ $\operatorname{Spec}\left(R^{(m)}\right)$ ) the set of all prime ideals (resp. $H$-invariant prime ideals) of $R^{(m)}$.

Recall (see section 3.2 ) that each prime ideal of $R^{(m)}$ is completely prime. If $m$ $\in \llbracket 2, \ldots, t \rrbracket$, we denote by

$$
\phi_{m}: \operatorname{Spec}\left(R^{(m+1)}\right) \rightarrow \operatorname{Spec}\left(R^{(m)}\right)
$$

the canonical injection defined in (4), section 4.3).

Moreover, if $m^{\prime} \leq m+1$ and if $\mathcal{P} \in \operatorname{Spec}\left(R^{(m+1)}\right)$, then $\mathcal{P}^{\prime}=\phi_{m^{\prime}} \circ \cdots \circ \phi_{m}(\mathcal{P})$ is called the canonical image of $\mathcal{P}$ in $\operatorname{Spec}\left(R^{\left(m^{\prime}\right)}\right)$. (If $m^{\prime}=m+1$, this canonical image is $\mathcal{P}$ itself.)

Let us now recall the main properties of the maps $\phi_{m}$.

- Denote by $\mathcal{P}^{(m+1)}$ any prime ideal in $\operatorname{Spec}\left(R^{(m+1)}\right)$ and set $\mathcal{P}^{(m)}$ $=\phi_{m}\left(\mathcal{P}^{(m+1)}\right)$.

(1) If $X_{m}^{(m+1)} \notin \mathcal{P}^{(m+1)}$, then $\mathcal{P}^{(m+1)} \cap S_{m}=\emptyset$ and

$$
\mathcal{P}^{(m)}=R^{(m)} \cap \mathcal{P}^{(m+1)} S_{m}^{-1} .
$$

In this case, $X_{m}^{(m)}=X_{m}^{(m+1)} \notin \mathcal{P}^{(m)}$.

(2) If $X_{m}^{(m+1)} \in \mathcal{P}^{(m+1)}$, there exists a unique algebra homomorphism $g: R^{(m)} \rightarrow R^{(m+1)} / \mathcal{P}^{(m+1)} \quad$ which satisfies $g\left(X_{i}^{(m)}\right)=X_{i}^{(m+1)}+$ $\mathcal{P}^{(m+1)}$ for all $i \in \llbracket 1, \ldots, t \rrbracket$, and $\mathcal{P}^{(m)}=\operatorname{Ker}(g)$. In this case, $X_{m}^{(m)}$ $=X_{m}^{(m+1)} \in \mathcal{P}^{(m)}$.

(3) For any $h \in H$, we have $h\left(\mathcal{P}^{(m)}\right)=\phi_{m}\left(h\left(\mathcal{P}^{(m+1)}\right)\right)($, lemma 5.5.5.) so that, by the injectivity of $\phi_{m}, \mathcal{P}^{(m+1)}$ is $H$-invariant if and only if $\mathcal{P}^{(m)}$ is $H$-invariant.

- A diagram $\Delta$ is said admissible (with respect to the reduced decomposition (1)) if there exists a prime ideal $\mathcal{P}$ of $R\left(=R^{(t+1)}\right)$ whose canonical image $\mathcal{P}^{(2)}$ in $\operatorname{Spec}(\bar{R})\left(=\operatorname{Spec}\left(R^{(2)}\right)\right)$ satisfies:

$$
\mathcal{P}^{(2)} \cap\left\{Z_{i} \mid 1 \leq i \leq t\right\}=\left\{Z_{i} \mid i \in \Delta\right\} .
$$


- Consider a diagram $\Delta$ and denote by $\mathcal{P}_{\Delta}^{(2)}=\left(\left\{Z_{i} \mid i \in \Delta\right\}\right)$ the ideal generated by the variables $Z_{i}$ with $i \in \Delta$. Then, by ([4], proposition 5.5.1.), we have

(1) $\mathcal{P}_{\Delta}^{(2)} \in H-\operatorname{Spec}\left(R^{(2)}\right)$.

(2) Conversely, for any $\mathcal{Q} \in H-\operatorname{Spec}\left(R^{(2)}\right)$, there exists a (unique) diagram $\Delta$ such that $\mathcal{Q}=\mathcal{P}_{\Delta}^{(2)}$, namely $\Delta=\left\{i \in \llbracket 1, t \rrbracket \mid Z_{i} \in \mathcal{Q}\right\}$.

- A diagram $\Delta$ is admissible if and only if there exists $\mathcal{P}_{\Delta} \in \operatorname{Spec}(R)(=$ $\operatorname{Spec}\left(R^{(t+1)}\right)$ ) such that

$$
\mathcal{P}_{\Delta}^{(2)}=\phi_{2} \circ \cdots \circ \phi_{t}\left(\mathcal{P}_{\Delta}\right) \text {. }
$$

(See 4], theorem 5.5.1. and observe that, since each $\phi_{i}$ is injective, $\mathcal{P}_{\Delta}$ is unique.)

- The map $\Delta \mapsto \mathcal{P}_{\Delta}$ is a bijection from the set of admissible diagrams onto the set $H-\operatorname{Spec}(R)\left(=H-\operatorname{Spec}\left(R^{(t+1)}\right)\right)$. In fact, if $\Delta$ is admissible, then $\mathcal{P}_{\Delta}$ is $H$-invariant because $\mathcal{P}_{\Delta}^{(2)}=\phi_{2} \circ \cdots \circ \phi_{t}\left(\mathcal{P}_{\Delta}\right)$ is. So, $\Delta \mapsto \mathcal{P}_{\Delta}$ is a map from the set of admissible diagrams into $H-\operatorname{Spec}(R)$. It is injective because the map $\Delta \mapsto \mathcal{P}_{\Delta}^{(2)}$ is injective. If $\mathcal{P} \in H-\operatorname{Spec}(R)$, then $\phi_{2} \circ \cdots \circ \phi_{t}(\mathcal{P}) \in H-\operatorname{Spec}\left(R^{(2)}\right)$. So $\phi_{2} \circ \cdots \circ \phi_{t}(\mathcal{P})=\mathcal{P}_{\Delta}^{(2)}$ with $\Delta$ an admissible diagram such that $\mathcal{P}=\mathcal{P}_{\Delta}$.

\section{New Results on $H$-InVARIant PRime ideals}

In this section, we consider an integer $m \in \llbracket 2, \ldots, t+1 \rrbracket$, and we denote by $\mathcal{P}^{(m)}$ an $H$-invariant prime ideal of $R^{(m)}$. We set $A^{(m)}=R^{(m)} / P^{(m)}$ and we observe that this algebra is a noetherian domain (since $R^{(m)}$ is noetherian and $P^{(m)}$ is completely prime). Set $D_{m}=\operatorname{Frac}\left(A^{(m)}\right)$ its division ring of fractions. Denote by $f_{m}: R^{(m)} \rightarrow A^{(m)}$ the canonical homomorphism and, for each $i \in \llbracket 1$, $\ldots, t \rrbracket$, set $x_{i}^{(m)}=f_{m}\left(X_{i}^{(m)}\right)$ the canonical image of $X_{i}^{(m)}$ in $A^{(m)}$. If $h$ is an element of the group $H$, we have $h\left(\mathcal{P}^{(m)}\right)=\mathcal{P}^{(m)}$ and, consequently, $h$ induces an automorphism of the algebra $A^{(m)}$, denoted $\bar{h}$, which satisfies $\bar{h} \circ f_{m}=f_{m} \circ h$. This automorphism can be extended to an automorphism, still denoted $\bar{h}$ of the division ring $D_{m}$.

4.1. A necessary and sufficient condition for $\mathcal{P}^{(m)}$ to be in $\operatorname{Im}\left(\phi_{m}\right)$. Assume that $m \leq t$ and recall (section 3.2) that $R^{(m)}$ is the $k$-algebra generated by the variables $X_{1}^{(m)}, \ldots, X_{t}^{(m)}$ subject to the relations (12):

$$
X_{j}^{(m)} X_{i}^{(m)}-\lambda_{j, i} X_{i}^{(m)} X_{j}^{(m)}=P_{j, i}^{(m)}\left(X_{i+1}^{(m)}, \ldots, X_{j-1}^{(m)}\right)
$$

for $i<j$, and with $P_{j, i}^{(m)}\left(X_{i+1}^{(m)}, \ldots, X_{j-1}^{(m)}\right) \in k\left\langle X_{i+1}^{(m)}, \ldots, X_{j-1}^{(m)}\right\rangle$.

Proposition 4.1.1. $\mathcal{P}^{(m)} \in \operatorname{Im}\left(\phi_{m}\right)$ if and only if one of two following conditions is satisfied:

a) $X_{m}^{(m)} \notin \mathcal{P}^{(m)}$.

b) $X_{m}^{(m)} \in \mathcal{P}^{(m)}$ and $\Theta^{(m)}\left(\delta_{m}^{(m+1)}\left(X_{i}^{(m+1)}\right)\right) \in \mathcal{P}^{(m)}$ for $1 \leq i \leq m-1$.

Proof. Assume that $\mathcal{P}^{(m)} \in \operatorname{Im}\left(\phi_{m}\right)$, so that $\mathcal{P}^{(m)}=\phi_{m}\left(\mathcal{P}^{(m+1)}\right)$ with $\mathcal{P}^{(m+1)}$ $\in \operatorname{Spec}\left(R^{(m+1)}\right)$, and assume that condition a) is not satisfied. This implies that $\mathcal{P}^{(m)}=\operatorname{ker}(g)$ where $g: R^{(m)} \rightarrow R^{(m+1)} / \mathcal{P}^{(m+1)}$ is the homomorphism which transforms each $X_{i}^{(m)}$ into $x_{i}^{(m+1)}=X_{i}^{(m+1)}+\mathcal{P}^{(m+1)}$. Consider $1 \leq i \leq m-1$. 
Recall that $\delta_{m}^{(m+1)}\left(X_{i}^{(m+1)}\right)=P_{m, i}^{(m+1)}\left(X_{i+1}^{(m+1)}, \ldots, X_{m-1}^{(m+1)}\right)$ and that $\Theta^{(m)}$ : $k\left\langle X_{1}^{(m+1)}, \ldots, X_{m-1}^{(m+1)}\right\rangle \rightarrow k\left\langle X_{1}^{(m)}, \ldots, X_{m-1}^{(m)}\right\rangle$ is the homomorphism which transforms each $X_{l}^{(m+1)}$ into $X_{l}^{(m)}$. Since $X_{m}^{(m)} \in \mathcal{P}^{(m)}$, we have $X_{m}^{(m+1)} \in \mathcal{P}^{(m+1)}([4$, proposition 4.3.1.) and so, $\delta_{m}^{(m+1)}\left(X_{i}^{(m+1)}\right) \in \mathcal{P}^{(m+1)}$. Now, we have

$$
\begin{aligned}
g\left(\Theta^{(m)}\left(\delta_{m}^{(m+1)}\left(X_{i}^{(m+1)}\right)\right)\right) & =g\left(\Theta^{(m)}\left(P_{m, i}^{(m+1)}\left(X_{i+1}^{(m+1)}, \ldots, X_{m-1}^{(m+1)}\right)\right)\right) \\
& =g\left(P_{m, i}^{(m+1)}\left(X_{i+1}^{(m)}, \ldots, X_{m-1}^{(m)}\right)\right)=P_{m, i}^{(m+1)}\left(x_{i+1}^{(m+1)}, \ldots, x_{m-1}^{(m+1)}\right) \\
& =P_{m, i}^{(m+1)}\left(X_{i+1}^{(m+1)}, \ldots, X_{m-1}^{(m+1)}\right)+\mathcal{P}^{(m+1)}=0 .
\end{aligned}
$$

This implies that $\left.\Theta^{(m)}\left(\delta_{m}^{(m+1)}\left(X_{i}^{(m+1)}\right)\right)\right) \in \operatorname{ker}(g)=\mathcal{P}^{(m)}$. If condition a) is satisfied, then $\mathcal{P}^{(m)} \in \operatorname{Im}\left(\phi_{m}\right)$ by ([4, lemma 4.3.1). Assume that condition b) is satisfied. So, if $1 \leq i \leq m-1$, we have, as previously, $P_{m, i}^{(m+1)}\left(X_{i+1}^{(m)}, \ldots, X_{m-1}^{(m)}\right)$ $=\Theta^{(m)}\left(\delta_{m}^{(m+1)}\left(X_{i}^{(m+1)}\right)\right) \in \mathcal{P}^{(m)}$. So, in $A^{(m)}=R^{(m)} / \mathcal{P}^{(m)}$, we have

$$
P_{m, i}^{(m+1)}\left(x_{i+1}^{(m)}, \ldots, x_{m-1}^{(m)}\right)=0 .
$$

Since $P_{m, i}^{(m)}=0$ (see section 3.2), we can write

$x_{m}^{(m)} x_{i}^{(m)}-\lambda_{m, i} x_{i}^{(m)} x_{m}^{(m)}=P_{m, i}^{(m)}\left(x_{i+1}^{(m)}, \ldots, x_{m-1}^{(m)}\right)=0=P_{m, i}^{(m+1)}\left(x_{i+1}^{(m)}, \ldots, x_{m-1}^{(m)}\right)$.

If $1 \leq i \leq j-1$ with $j \neq m$, we have (see section 3.2)

$$
x_{j}^{(m)} x_{i}^{(m)}-\lambda_{j, i} x_{i}^{(m)} x_{j}^{(m)}=P_{j, i}^{(m)}\left(x_{i+1}^{(m)}, \ldots, x_{j-1}^{(m)}\right)=P_{j, i}^{(m+1)}\left(x_{i+1}^{(m)}, \ldots, x_{j-1}^{(m)}\right) .
$$

So, by the universal property of algebras defined by generators and relations, there exists a (unique) homomorphism $\epsilon: R^{(m+1)} \rightarrow A^{(m)}$ which transforms each $X_{l}^{(m+1)}$ into $x_{l}^{(m)}$. This homomorphism is surjective, and its kernel $\operatorname{ker}(\epsilon)=$ $\mathcal{P}^{(m+1)}$ is a prime ideal of $R^{(m+1)}$. We observe that, since $X_{m}^{(m)} \in \mathcal{P}^{(m)}$, we have $X_{m}^{(m+1)} \in \mathcal{P}^{(m+1)}$, and that $\epsilon$ induces an isomorphism

$$
\bar{\epsilon}: R^{(m+1)} / \mathcal{P}^{(m+1)} \rightarrow A^{(m)}=R^{(m)} / \mathcal{P}^{(m)}
$$

which transforms each $x_{l}^{(m+1)}$ into $x_{l}^{(m)}$. Recall that $f_{m}: R^{(m)} \rightarrow R^{(m)} / \mathcal{P}^{(m)}$ denotes the canonical homomorphism.

So, $g=(\bar{\epsilon})^{-1} \circ f_{m}: R^{(m)} \rightarrow R^{(m+1)} / \mathcal{P}^{(m+1)}$ is the homomorphism which transforms each $X_{l}^{(m)}$ into $x_{l}^{(m+1)}$. As $\operatorname{ker}(g)=\operatorname{ker}\left(f_{m}\right)=\mathcal{P}^{(m)}$, we conclude that $\mathcal{P}^{(m)}$ $=\phi_{m}\left(\mathcal{P}^{(m+1)}\right)$.

Corollary 4.1.1. Assume $2 \leq m \leq t+1$ and consider some $\mathcal{Q} \in \operatorname{Spec}\left(R^{(m)}\right)$.

Assume that $X_{m}^{(m)} \notin \mathcal{Q}, \ldots, X_{t}^{(m)} \notin \mathcal{Q}$.

Then there exists $\mathcal{P} \in \operatorname{Spec}(R)$ such that $\mathcal{Q}$ is the canonical image of $\mathcal{P}$ in $\operatorname{Spec}\left(R^{(m)}\right)$. Moreover, if $\mathcal{P}^{(2)}$ is the canonical image of $\mathcal{P}$ in $\operatorname{Spec}\left(R^{(2)}\right)$, then

$$
\mathcal{P}^{(2)} \cap\left\{Z_{m}, \ldots, Z_{t}\right\}=\emptyset .
$$

Proof. We prove this by decreasing induction on $m$.

- If $m=t+1$, we have $\mathcal{Q} \in \operatorname{Spec}(R)$ and $\mathcal{P}=\mathcal{Q}$ satisfies the required properties. 
- Assume $2 \leq m \leq t$. By proposition 4.1.1, we have $\mathcal{Q}=\phi_{m}\left(\mathcal{Q}^{\prime}\right)$ with $\mathcal{Q}^{\prime} \in$ $\operatorname{Spec}\left(R^{(m+1)}\right)$. Since $X_{m}^{(m)} \notin \mathcal{Q}$, we have (see section 3.3) $X_{m}^{(m+1)} \notin \mathcal{Q}^{\prime}$ and, if we set $S_{m}=\left\{\left(X_{m}^{(m+1)}\right)^{d} \mid d \in \mathbb{N}\right\}$, then

$$
\mathcal{Q}=R^{(m)} \cap \mathcal{Q}^{\prime} S_{m}^{-1} .
$$

Assume $X_{j}^{(m+1)} \in \mathcal{Q}^{\prime}$ with $j \geq m$. Since $X_{j}^{(m+1)}=X_{j}^{(m)}$ (see section 3.2), we have $X_{j}^{(m)} \in R^{(m)} \cap \mathcal{Q}^{\prime} \subseteq R^{(m)} \cap \mathcal{Q}^{\prime} S_{m}^{-1}=\mathcal{Q}$, which is false. So, we have:

$-X_{m}^{(m+1)} \notin \mathcal{Q}^{\prime}$.

- $X_{m+1}^{(m+1)} \notin \mathcal{Q}^{\prime}, \ldots, X_{t}^{(m+1)} \notin \mathcal{Q}^{\prime}$ and, by the induction assumption, there exists $\mathcal{P} \in \operatorname{Spec}(R)$ such that $\mathcal{Q}^{\prime}$ is the canonical image of $\mathcal{P}$ in $\operatorname{Spec}\left(R^{(m+1)}\right)$. This implies that $\mathcal{Q}=\phi_{m}\left(\mathcal{Q}^{\prime}\right)$ is the canonical image of $\mathcal{P}$ in $\operatorname{Spec}\left(R^{(m)}\right)$. Moreover, again by the induction assumption, we have

$$
\mathcal{P}^{(2)} \cap\left\{Z_{m+1}, \ldots, Z_{t}\right\}=\emptyset .
$$

As $X_{m}^{(m+1)} \notin \mathcal{Q}^{\prime}$, we know ([4], proposition 5.2.1) that $Z_{m} \notin \mathcal{P}^{(2)}$.

4.2. Some properties of $A^{(m)}$. For each integer $j \in \llbracket 2, \ldots, m-1 \rrbracket$, we set $\mathcal{P}^{(j)}$ $=\phi_{j} \circ \cdots \circ \phi_{m-1}\left(\mathcal{P}^{(m)}\right)$. Let us, first, construct in $A^{(m)}$, a new version of the deleting derivations algorithm.

Proposition 4.2.1. For each integer $j \in \llbracket 2, \ldots, m-1 \rrbracket$, there exists a sequence $\left(x_{1}^{(j)}, \ldots, x_{t}^{(j)}\right)$ in $D_{m}=\operatorname{Frac}\left(A^{(m)}\right)$ and a subalgebra $A^{(j)}=k\left\langle x_{1}^{(j)}, \ldots, x_{t}^{(j)}\right\rangle$ of $D_{m}$ which satisfy the following properties:

(1) If $l$ and $i$ are in $\llbracket 1, \ldots, t \rrbracket$, we have $\overline{h_{l}}\left(x_{i}^{(j)}\right)=\lambda_{l, i} x_{i}^{(j)}, \overline{h_{l}}\left(A^{(j)}\right)=A^{(j)}$ and $\overline{h_{l}}$ induces, by restriction, an automorphism (still denoted $\left.\overline{h_{l}}\right)$ of $A^{(j)}$.

(2) Choose $l \in \llbracket 1, \ldots, t \rrbracket$ and denote by $d_{l}^{(j)}$ the left $\overline{h_{l}}$-derivation of $A^{(j)}$ associated to $x_{l}^{(j)}\left(d_{l}^{(j)}(a)=x_{l}^{(j)} a-\overline{h_{l}}(a) x_{l}^{(j)}\right.$ for all $\left.a \in A^{(j)}\right)$. Then $d_{l}^{(j)}\left(k\left\langle x_{1}^{(j)}, \ldots, x_{l-1}^{(j)}\right\rangle\right) \subset k\left\langle x_{1}^{(j)}, \ldots, x_{l-1}^{(j)}\right\rangle$ and $d_{l}^{(j)}$ is locally nilpotent on $k\left\langle x_{1}^{(j)}, \ldots, x_{l-1}^{(j)}\right\rangle$.

(3) There exists a unique homomorphism $f_{j}: R^{(j)} \rightarrow A^{(j)}$ which transforms each $X_{i}^{(j)}$ into $x_{i}^{(j)}$. $f_{j}$ is surjective and $\operatorname{ker}\left(f_{j}\right)=\mathcal{P}^{(j)}$.

(4) If $l \in \llbracket 1, \ldots, t \rrbracket$, we have $d_{l}^{(j)} \circ f_{j}=f_{j} \circ \delta_{l}^{(j)}$ and $\overline{h_{l}} \circ f_{j}=f_{j} \circ h_{l}$.

(5) If $l, e \in \llbracket 1, \ldots, t \rrbracket$, we have $\overline{h_{l}} \circ \overline{h_{e}}=\overline{h_{e}} \circ \overline{h_{l}}$ and $\overline{h_{l}} \circ d_{e}^{(j)}=\lambda_{l, e} d_{e}^{(j)} \circ \overline{h_{l}}$.

(6) If $x_{j}^{(j+1)}=0$, then $x_{i}^{(j)}=x_{i}^{(j+1)}$ for each $i \in \llbracket 1, \ldots, t \rrbracket$.

(7) If $x_{j}^{(j+1)} \neq 0$, and $i \in \llbracket 1, \ldots, t \rrbracket$, then

(a) $j \leq i \Rightarrow x_{i}^{(j)}=x_{i}^{(j+1)}$. In particular, we have $x_{j}^{(j)}=x_{j}^{(j+1)}$. 
(b) $i<j \Rightarrow x_{i}^{(j)}=x_{i}^{(j+1)}+\sum_{d=1}^{+\infty} c_{d}^{(j+1)}\left(x_{j}^{(j+1)}\right)^{-d}$ with

$$
c_{d}^{(j+1)}=\frac{\left(1-q_{j}\right)^{-d}}{[d] !_{q_{j}}} \lambda_{j, i}^{-d}\left(d_{j}^{(j+1)}\right)^{d}\left(x_{i}^{(j+1)}\right) .
$$

(We observe that, since $k\left\langle x_{1}^{(j+1)}, \ldots, x_{j-1}^{(j+1)}\right\rangle$ is $d_{j}^{(j+1)}$-stable, we have $c_{d}^{(j+1)} \in k\left\langle x_{1}^{(j+1)}, \ldots, x_{j-1}^{(j+1)}\right\rangle$ and, since $d_{j}^{(j+1)}$ is locally nilpotent on $k\left\langle x_{1}^{(j+1)}, \ldots, x_{j-1}^{(j+1)}\right\rangle$, only finitely many $c_{d}^{(j+1)}$ are nonzero. $)$

Proof. The existence of variables $x_{i}^{(j)}(i \in \llbracket 1, \ldots, t \rrbracket, j \in \llbracket 2, \ldots, m-1 \rrbracket)$ and algebras $A^{(j)}=k\left\langle x_{1}^{(j)}, \ldots, x_{t}^{(j)}\right\rangle$ satisfying conditions (3), (6) and (7) is a consequence of [4, Propositions 5.4.1, 5.4.2], replacing $R$ by $R^{(m)}$ and $\mathcal{P}$ by $\mathcal{P}^{(m)}$.

Using (3), we observe that, if $\overline{h_{l}} \circ f_{j}=f_{j} \circ h_{l}$ (as a homomorphism from $R^{(j)}$ towards $\left.D^{(m)}\right)$ holds for any $l$, then properties (1), (2), (4) and (5) are all satisfied. In fact,

1. For all $l$ and $i$ in $\llbracket 1, \ldots, t \rrbracket$, we have $\overline{h_{l}}\left(x_{i}^{(j)}\right)=\overline{h_{l}} \circ f_{j}\left(X_{i}^{(j)}\right)=f_{j} \circ h_{l}\left(X_{i}^{(j)}\right)$ $=\lambda_{l, i} f_{j}\left(X_{i}^{(j)}\right)=\lambda_{l, i} x_{i}^{(j)}$. This immediately implies that $\overline{h_{l}}\left(A^{(j)}\right)=A^{(j)}$ and $\overline{h_{l}}$ induces, by restriction, an automorphism of $A^{(j)}$.

4. If $P \in R^{(j)}$, we have $d_{l}^{(j)}\left(f_{j}(P)\right)=x_{l}^{(j)} f_{j}(P)-\overline{h_{l}}\left(f_{j}(P)\right) x_{l}^{(j)}=f_{j}\left(X_{l}^{(j)} P-\right.$ $\left.h_{l}(P) X_{l}^{(j)}\right)=f_{j} \circ \delta_{l}^{(j)}(P)$. This proves that $d_{l}^{(j)} \circ f_{j}=f_{j} \circ \delta_{l}^{(j)}$.

2. By (4), we have $d_{l}^{(j)}\left(k\left\langle x_{1}^{(j)}, \ldots, x_{l-1}^{(j)}\right\rangle\right)=d_{l}^{(j)}\left(f_{j}\left(k\left\langle X_{1}^{(j)}, \ldots, X_{l-1}^{(j)}\right\rangle\right)\right)=$ $f_{j}\left(\delta_{l}^{(j)}\left(k\left\langle X_{1}^{(j)}, \ldots, X_{l-1}^{(j)}\right\rangle\right)\right) \subset f_{j}\left(k\left\langle X_{1}^{(j)}, \ldots, X_{l-1}^{(j)}\right\rangle\right)=k\left\langle x_{1}^{(j)}, \ldots, x_{l-1}^{(j)}\right\rangle$. Moreover, if $a \in k\left\langle x_{1}^{(j)}, \ldots, x_{l-1}^{(j)}\right\rangle$, we can write $a=f_{j}(P)$ with $P \in$ $k\left\langle X_{1}^{(j)}, \ldots, X_{l-1}^{(j)}\right\rangle$. Since $\delta_{l}^{(j)}$ is locally nilpotent on $k\left\langle X_{1}^{(j)}, \ldots, X_{l-1}^{(j)}\right\rangle$, we have $\left(\delta_{l}^{(j)}\right)^{r}(P)=0$ for some $r \in \mathbb{N}$, and $\left(d_{l}^{(j)}\right)^{r}(a)=\left(d_{l}^{(j)}\right)^{r} \circ f_{j}(P)=$ $f_{j} \circ\left(\delta_{l}^{(j)}\right)^{r}(P)=0$. So, $d_{l}^{(j)}$ is locally nilpotent on $k\left\langle x_{1}^{(j)}, \ldots, x_{l-1}^{(j)}\right\rangle$.

5. If $i$ in $\llbracket 1, \ldots, t \rrbracket$, we have $\overline{h_{l}} \circ \overline{h_{e}}\left(x_{i}^{(j)}\right)=\lambda_{l, i} \lambda_{e, i} x_{i}^{(j)}=\overline{h_{e}} \circ \overline{h_{l}}\left(x_{i}^{(j)}\right)$ and, since $A^{(j)}$ is generated by the elements $x_{i}^{(j)}$, we have $\overline{h_{l}} \circ \overline{h_{e}}=\overline{h_{e}} \circ \overline{h_{l}}$. Consider two elements $a, b$ in $A^{(j)}$ such that the maps $\overline{h_{l}} \circ d_{e}^{(j)}$ and $\lambda_{l, e} d_{e}^{(j)} \circ \overline{h_{l}}$ coincide on $a$ and $b$. Then, we have $\overline{h_{l}} \circ d_{e}^{(j)}(a b)=\overline{h_{l}}\left(d_{e}^{(j)}(a) b+\overline{h_{e}}(a) d_{e}^{(j)}(b)\right)=$ $\lambda_{l, e} d_{e}^{(j)}\left(\overline{h_{l}}(a)\right) \overline{h_{l}}(b)+\lambda_{l, e} \overline{h_{e}}\left(\overline{h_{l}}(a)\right) d_{e}^{(j)}\left(\overline{h_{l}}(b)\right)=\lambda_{l, e} d_{e}^{(j)} \circ \overline{h_{l}}(a b)$. So, we just have to prove that $\overline{h_{l}} \circ d_{e}^{(j)}$ and $\lambda_{l, e} d_{e}^{(j)} \circ \overline{h_{l}}$ coincide on each $x_{i}^{(j)}$. Now, $\overline{h_{l}} \circ d_{e}^{(j)}\left(x_{i}^{(j)}\right)=\overline{h_{l}}\left(x_{e}^{(j)} x_{i}^{(j)}-\lambda_{e, i} x_{i}^{(j)} x_{e}^{(j)}\right)=\lambda_{l, e} \lambda_{l, i}\left(x_{e}^{(j)} x_{i}^{(j)}-\lambda_{e, i} x_{i}^{(j)} x_{j}^{(j)}\right)$ $=\lambda_{l, e} \lambda_{l, i} d_{e}^{(j)}\left(x_{i}^{(j)}\right)=\lambda_{l, e} d_{e}^{(j)}\left(\overline{h_{l}}\left(x_{i}^{(j)}\right)\right)$. So we can conclude that $\overline{h_{l}} \circ d_{e}^{(j)}$ $=\lambda_{l, e} d_{e}^{(j)} \circ \overline{h_{l}}$.

Now, it remains to verify that, for any $l$ in $\llbracket 1, \ldots, t \rrbracket$ and for any $j \in \llbracket 2, m \rrbracket$, we have $\overline{h_{l}} \circ f_{j}=f_{j} \circ h_{l}$. As $h_{l}\left(X_{i}^{(j)}\right)=\lambda_{l, i} X_{i}^{(j)}$, it is enough to check that $\overline{h_{l}}\left(x_{i}^{(j)}\right)=\lambda_{l, i} x_{i}^{(j)}$ for each $i$.

If $j=m$, the constructions of $f_{m}$ and of the variables $x_{i}^{(m)}$ (see the beginning of section 4) imply straightaway the equality $\bar{h} \circ f_{m}=f_{m} \circ h$ for any $h$ in $H$.

Assume that $j \in \llbracket 2, m-1 \rrbracket$ and $\overline{h_{l}} \circ f_{j+1}=f_{j+1} \circ h_{l}$ for any $l \in \llbracket 1, \ldots, t \rrbracket$. So properties (1) to (7) hold for $j+1$.

Now, consider $l$ and $i$ in $\llbracket 1, \ldots, t \rrbracket$. 
If $j \leq i$, by property (4) at the rank $j+1$, we have $\overline{h_{l}}\left(x_{i}^{(j)}\right)=\overline{h_{l}}\left(x_{i}^{(j+1)}\right)=$ $\lambda_{l, i} x_{i}^{(j+1)}=\lambda_{l, i} x_{i}^{(j)}$.

If $i<j$, it follows from property (5) at the rank $j+1$ that

$$
\overline{h_{l}} \circ d_{j}^{(j+1)}=\lambda_{l, j} d_{j}^{(j+1)} \circ \overline{h_{l}} .
$$

So, for each $d \in \mathbb{N}$, we have

$$
\begin{aligned}
\overline{h_{l}}\left(c_{d}^{(j+1)}\right) & =\overline{h_{l}}\left(\frac{\left(1-q_{j}\right)^{-d}}{[d] !_{q_{j}}} \lambda_{j, i}^{-d}\left(d_{j}^{(j+1)}\right)^{d}\left(x_{i}^{(j+1)}\right)\right) \\
& =\frac{\left(1-q_{j}\right)^{-d}}{[d] !_{q_{j}}} \lambda_{j, i}^{-d} \lambda_{l, j}^{d}\left(d_{j}^{(j+1)}\right)^{d} \circ \overline{h_{l}}\left(x_{i}^{(j+1)}\right)=\lambda_{l, i} \lambda_{l, j}^{d} c_{d}^{(j+1)} .
\end{aligned}
$$

From this, $\overline{h_{l}}\left(c_{d}^{(j+1)}\left(x_{j}^{(j+1)}\right)^{-d}\right)=\lambda_{l, i} c_{d}^{(j+1)}\left(x_{j}^{(j+1)}\right)^{-d}$, which implies that $\overline{h_{l}}\left(x_{i}^{(j)}\right)$ $=\lambda_{l, i} x_{i}^{(j)}$.

Recall (see section 3.3) that, since $\mathcal{P}^{(m)}$ is $H$-invariant, each $\mathcal{P}^{(j)}(2 \leq j \leq m$ ) is $H$-invariant. In particular, $\mathcal{P}^{(2)}$ is $H$-invariant. Set

$$
\Delta=\left\{i \in \llbracket 1, \ldots, t \| \mid Z_{i} \in \mathcal{P}^{(2)}\right\}=\left\{j_{1}<\cdots<j_{s}\right\} \text { (unless this set is empty) }
$$

and

$$
\bar{\Delta}=\llbracket 1, \ldots, t \rrbracket \backslash \Delta=\left\{l_{1}<\cdots<l_{e}\right\} \text { (unless this set is empty). }
$$

Recall that $\mathcal{P}^{(2)}=\mathcal{P}_{\Delta}^{(2)}$ (see section 3.3), $\bar{R}=R^{(2)}$ and set $\bar{A}=A^{(2)}$. For each $i \in \llbracket 1, \ldots, t \rrbracket$, set $z_{i}=x_{i}^{(2)}$ and observe that $f_{2}: \bar{R} \rightarrow \bar{A}$ transforms each $Z_{i}=$ $X_{i}^{(2)}$ into $z_{i}$. So, we can also describe $\Delta$ and $\bar{\Delta}$ as follows:

$$
\begin{aligned}
& \Delta=\left\{i \in \llbracket 1, \ldots, t \rrbracket \mid z_{i}=0\right\}, \\
& \bar{\Delta}=\left\{i \in \llbracket 1, \ldots, t \rrbracket \mid z_{i} \neq 0\right\} .
\end{aligned}
$$

Observation 4.2.1. Assume that $\bar{\Delta}$ is empty. Then each $x_{i}^{(m)}(i \in \llbracket 1, \ldots, t \rrbracket)$ is zero.

Proof. If $\bar{\Delta}$ is empty, we have $x_{j}^{(j+1)}=z_{j}=0$ for each $j \in \llbracket 1, \ldots, m-1 \rrbracket$. By (proposition 4.2.1, (6)), this implies that, if $i \in \llbracket 1, \ldots, t \rrbracket$ and $j \in \llbracket 1, \ldots, m-1 \rrbracket$, we have $x_{i}^{(j+1)}=x_{i}^{(j)}$. So, each $x_{i}^{(m)}=x_{i}^{(2)}=z_{i}=0$.

Until the end of section 4.2 , we assume that $\bar{\Delta}$ is nonempty. So, we have:

Proposition 4.2.2. $\quad$ (1) $\bar{A}=k\left\langle z_{l_{1}}, \ldots, z_{l_{e}}\right\rangle$.

(2) If $1 \leq i<d \leq e$, we have $z_{l_{d}} z_{l_{i}}=\lambda_{l_{d}, l_{i}} z_{l_{i}} z_{l_{d}}$.

(3) $z_{l_{1}}, \ldots, z_{l_{e}}$ are all nonzero and the Laurent (ordered) monomials $z^{\underline{a}}=$ $z_{l_{1}}^{a_{1}} \ldots z_{l_{e}}^{a_{e}}\left(\underline{a}=\left(a_{1}, \ldots, a_{e}\right) \in \mathbb{Z}^{e}\right)$ are k-linearly independent.

Proof. (1) $\bar{A}=k\left\langle z_{1}, \ldots, z_{t}\right\rangle$ (see proposition 4.2. 1) and, for $i \notin\left\{l_{1}, \ldots, l_{e}\right\}$, we have $i \in \Delta \Rightarrow Z_{i} \in \mathcal{P}^{(2)}=\operatorname{ker}\left(f_{2}\right) \Rightarrow z_{i}=0$.

(2) We know (section 3.2) that $Z_{l_{d}} Z_{l_{i}}=\lambda_{l_{d}, l_{i}} Z_{l_{i}} Z_{l_{d}}$. If we transform by $f_{2}$, we obtain the required equality.

(3) Denote by $\widehat{R}=k\left\langle Z_{l_{1}}, \ldots, Z_{l_{e}}\right\rangle$ the subalgebra of $\bar{R}$ generated by $Z_{l_{1}}, \ldots, Z_{l_{e}}$. By the property (1), $f_{2}$ induces (by restriction) a surjective homomorphism $\widehat{f_{2}}: \widehat{R} \rightarrow \bar{A}$ and $\operatorname{ker}\left(\widehat{f}_{2}\right)=\widehat{R} \cap \mathcal{P}^{(2)}$. As each $P \in \mathcal{P}^{(2)}=\mathcal{P}_{\Delta}^{(2)}=$ $\left(\left\{Z_{j_{1}}, \ldots, Z_{j_{s}}\right\}\right)$ is a linear combination of monomials in which at least one 
of the variables $Z_{j_{i}}$ with $1 \leq i \leq s$ appears, this intersection is reduced to zero and then, $\widehat{f}_{2}$ is an isomorphism which transforms each $Z_{l_{i}}$ into $z_{l_{i}}$. As the ordered monomials in $Z_{l_{1}}, \ldots, Z_{l_{e}}$ are linearly independent, we have the same property for the ordered monomials in $z_{l_{1}}, \ldots, z_{l_{e}}$. This easily implies that $z_{l_{1}}, \ldots, z_{l_{e}}$ are nonzero and that the Laurent ordered monomials in $z_{l_{1}}, \ldots, z_{l_{e}}$ are also linearly independent.

4.3. Each $x_{i}^{(m)}$ is a Laurent polynomial in $z_{l_{1}}, \ldots, z_{l_{e}}$. The conventions are the same as in section 4.2 and we still assume that $\bar{\Delta}$ is nonempty.

Let us consider some $i$ in $\llbracket 1, \ldots, t \rrbracket$.

If $u \in D_{m}$ and if $\gamma \in \mathbb{Z} \Pi$, we say (as in section 3.2) that $u$ is homogeneous of degree $\gamma$ if $\overline{h_{\rho}}(u)=q^{-(\rho, \gamma)} u$ for all $\rho$ in $\mathbb{Z} \Pi$. Since $q$ is not a root of unity and $V$ is spanned by $\Pi$, the degree of a nonzero homogeneous element is uniquely defined. Moreover, we immediately have the following properties:

- If $u_{1}, \ldots, u_{r}$ are homogeneous of same degree $\gamma$, then any linear combination of $u_{1}, \ldots, u_{r}$ (with coefficients in $k$ ) is homogeneous of degree $\gamma$.

- If $u_{1}$ is homogeneous of degree $\gamma_{1}$ and $u_{2}$ is homogeneous of degree $\gamma_{2}$, then $u_{1} u_{2}$ is homogeneous of degree $\gamma_{1}+\gamma_{2}$.

- Likewise, if $u$ is nonzero and homogeneous of degree $\gamma$, then $u^{-1}$ is homogeneous of degree $-\gamma$.

- So, if $u_{1}, \ldots, u_{r}$ are nonzero and homogeneous of degrees $\gamma_{1}, \ldots, \gamma_{r}$, respectively, if $\underline{a}=\left(a_{1}, \ldots, a_{r}\right) \in \mathbb{Z}^{r}$, then $u^{\underline{a}}:=u_{1}^{a_{1}} \ldots u_{r}^{a_{r}}$ is homogeneous of degree $a_{1} \gamma_{1}+\cdots+a_{r} \gamma_{r}$.

Lemma 4.3.1. Consider some $j$ in $\llbracket 2, \ldots, m \rrbracket$.

(1) If $j \leq i+1$, then $x_{i}^{(j)}=z_{i}$. In particular, $x_{i}^{(i+1)}=z_{i}$.

(2) If $1 \leq i<l \leq t$, then $x_{l}^{(j)} x_{i}^{(j)}-\lambda_{l, i} x_{i}^{(j)} x_{l}^{(j)}=d_{l}^{(j)}\left(x_{i}^{(j)}\right)=f_{j}\left(P_{l, i}^{(j)}\right) \in$ $k\left\langle x_{i+1}^{(j)}, \ldots, x_{l-1}^{(j)}\right\rangle$. Moreover, if $l \geq j$ or $l=i+1$, then $d_{l}^{(j)}\left(x_{i}^{(j)}\right)=0$.

(3) If $U \in R^{(j)}$ is homogeneous of degree $\gamma$, then $f_{j}(U)$ is homogeneous of same degree $\gamma$. In particular, $x_{i}^{(j)}$ is homogeneous of degree $\beta_{i}$.

(4) If $j \leq l \leq t$ and $u \in k\left\langle x_{1}^{(j)}, \ldots, x_{l-1}^{(j)}\right\rangle$, then $z_{l} u=\overline{h_{l}}(u) z_{l}$.

Proof. (1) By proposition 4.2.1, we have $x_{i}^{(i+1)}=x_{i}^{(i)}=\cdots=x_{i}^{(2)}=z_{i}$.

(2) By proposition 4.2.1, we have $x_{l}^{(j)} x_{i}^{(j)}-\lambda_{l, i} x_{i}^{(j)} x_{l}^{(j)}=x_{l}^{(j)} x_{i}^{(j)}-\overline{h_{l}}\left(x_{i}^{(j)}\right) x_{l}^{(j)}$ $=d_{l}^{(j)}\left(x_{i}^{(j)}\right)=d_{l}^{(j)} \circ f_{j}\left(X_{i}^{(j)}\right)=f_{j} \circ \delta_{l}^{(j)}\left(X_{i}^{(j)}\right)$ and we know (see section 3.2) that $\delta_{l}^{(j)}\left(X_{i}^{(j)}\right)=P_{l, i}^{(j)} \in k\left\langle X_{i+1}^{(j)}, \ldots, X_{l-1}^{(j)}\right\rangle$. Moreover, if $l \geq j$ or $l$ $=i+1$, we know (see section 3.2 ) that $P_{l, i}^{(j)}=0$. Transforming by $f_{j}$, we get the required result.

(3) Consider $\rho$ in $\mathbb{Z} \Pi$. By proposition 4.2.1, we have $\overline{h_{\rho}}\left(f_{j}(U)\right)=f_{j}\left(h_{\rho}(U)\right)$ $=q^{-(\rho, \gamma)} f_{j}(U)$.

(4) By (1), we have $z_{l}=x_{l}^{(j)}$. So, $z_{l} u-\overline{h_{l}}(u) z_{l}=x_{l}^{(j)} u-\overline{h_{l}}(u) x_{l}^{(j)}=d_{l}^{(j)}(u)$. By (2), if $i<l$, we have $d_{l}^{(j)}\left(x_{i}^{(j)}\right)=0$ (since $j \leq l$ ). This implies that $d_{l}^{(j)}$ is zero on $k\left\langle x_{1}^{(j)}, \ldots, x_{l-1}^{(j)}\right\rangle$ and, in particular, $d_{l}^{(j)}(u)=0$.

Lemma 4.3.2. Consider some $j$ in $\llbracket 2, \ldots, m \rrbracket$.

(1) If $j \leq l_{1}$, then $x_{i}^{(j)}=z_{i}$. 
(2) Assume that $l_{1}<j$ and denote by $d$ the greatest integer such that $l_{d}<j$ $(1 \leq d \leq p)$. Then $x_{i}^{(j)}=x_{i}^{\left(l_{d}+1\right)}$.

Proof.

2. We prove this by induction on $j$. If $j=l_{d}+1$, there is nothing to prove. Assume $j>l_{d}+1$ and set $j^{\prime}=j-1>l_{d}$. By lemma 4.3.1, we have $x_{j^{\prime}}^{\left(j^{\prime}+1\right)}=z_{j^{\prime}}=0\left(\right.$ since $\left.j^{\prime} \in \Delta\right)$. So, by proposition $4.2 .1, x_{i}^{(j)}=x_{i}^{\left(j^{\prime}+1\right)}=$ $x_{i}^{\left(j^{\prime}\right)}=x_{i}^{\left(l_{d}+1\right)}$ (by the induction assumption).

1. The proof is the same (observing that there is nothing to prove if $j=2$ ).

Now, assume that $l_{1}<m$ and denote by $p$ the greatest integer such that $l_{p}<m$.

Proposition 4.3.1. Consider some $j$ in $\llbracket 2, \ldots, m \rrbracket$ and assume that $i<j$.

(1) If $i<j \leq l_{1}$, then $x_{i}^{(j)}=z_{i}=0$. Assume that $l_{1}<j$ and denote by $d$ the greatest integer such that $l_{d}<j(1 \leq d \leq p)$.

(2) (a) If $l_{d} \leq i<j$, then $x_{i}^{(j)}=z_{i}$.

(b) If $i<l_{d}<j$, then

$$
x_{i}^{(j)}=x_{i}^{\left(l_{d}\right)}+Q_{1} z_{l_{d}}^{-1}+\cdots+Q_{K} z_{l_{d}}^{-K}
$$

with $K \geq 1$ and:

- If $d=1$, then each $Q_{l} \in k$.

- If $d>1$ and $i \geq l_{d-1}$, then each $Q_{l} \in k$.

- If $d>1$ and $i<l_{d-1}$, then each $Q_{l} \in k\left\langle x_{i+1}^{\left(l_{d}\right)}, \ldots, x_{l_{d-1}}^{\left(l_{d}\right)}\right\rangle$.

Proof. (1) By (lemma 4.3.2, (1)), we have $x_{i}^{(j)}=z_{i}$ and, since $i<l_{1}$, we have $i \in \Delta$, so that $z_{i}=0$.

(2) (a) By (lemma 4.3.2, (2)), we have $x_{i}^{(j)}=x_{i}^{\left(l_{d}+1\right)}$ and, since $l_{d}+1 \leq i$, this is also equal to $z_{i}$.

(b) We prove this result by decreasing induction on $i$.

As in (2)(a), we have $x_{i}^{(j)}=x_{i}^{\left(l_{d}+1\right)}$ and, since $x_{l_{d}}^{\left(l_{d}+1\right)}=z_{l_{d}} \neq 0$, it follows from (proposition 4.2.1, (7(b)) that

$$
x_{i}^{(j)}=x_{i}^{\left(l_{d}\right)}+P_{1} z_{l_{d}}^{-1}+\cdots+P_{L} z_{l_{d}}^{-L}
$$

with $L \geq 1$ and each $P_{l}=\mu_{l}\left(d_{l_{d}}^{\left(l_{d}+1\right)}\right)^{l}\left(x_{i}^{\left(l_{d}+1\right)}\right)\left(\mu_{l} \in k^{*}\right)$. So, by lemma 4.3.1, each $P_{l} \in k\left\langle x_{i+1}^{\left(l_{d}+1\right)}, \ldots, x_{l_{d}-1}^{\left(l_{d}+1\right)}\right\rangle=k\left\langle x_{i+1}^{(j)}, \ldots, x_{l_{d}-1}^{(j)}\right\rangle$ (by lemma 4.3.2).

Assume that $i=l_{d}-1$. Then, by lemma 4.3.1, we have $d_{l_{d}}^{\left(l_{d}+1\right)}\left(x_{i}^{\left(l_{d}+1\right)}\right)$ $=0$. This implies that $P_{l}=0$ for each $l$. So, $x_{i}^{(j)}=x_{i}^{\left(l_{d}\right)}$ and the proof is over.

Now, assume that $i<l_{d}-1$ and, for $i+1 \leq h \leq l_{d}-1$,

$$
x_{h}^{(j)}=x_{h}^{\left(l_{d}\right)}+Q_{1, h} z_{l_{d}}^{-1}+\cdots+Q_{M, h} z_{l_{d}}^{-M}
$$

with $M \geq 1$, and:

- each $Q_{l, h} \in k$ if $d=1$,

- $Q_{l, h} \in k$ if $d>1$ and $h \geq l_{d-1}$,

- $Q_{l, h} \in k\left\langle x_{h+1}^{\left(l_{d}\right)}, \ldots x_{l_{d-1}}^{\left(l_{d}\right)}\right\rangle$ if $d>1$ and $h<l_{d-1}$. 
This implies the following results:

- If $d=1$, then each $x_{h}^{(j)} \in k\left\langle x_{h}^{\left(l_{d}\right)}, z_{l_{d}}^{-1}\right\rangle\left(i+1 \leq h \leq l_{d}-1\right)$.

- If $d>1$ and $l_{d-1} \leq h \leq l_{d}-1$, then $x_{h}^{(j)} \in k\left\langle x_{h}^{\left(l_{d}\right)}, z_{l_{d}}^{-1}\right\rangle$.

- If $d>1$ and $i+1 \leq h<l_{d-1}$, then $x_{h}^{(j)} \in k\left\langle x_{h}^{\left(l_{d}\right)}, x_{h+1}^{\left(l_{d}\right)}, \ldots\right.$, $\left.x_{l_{d-1}}^{\left(l_{d}\right)}, z_{l_{d}}^{-1}\right\rangle$.

So, it turns out that each $P_{l} \in k\left\langle x_{i+1}^{\left(l_{d}\right)}, \ldots, x_{l_{d}-1}^{\left(l_{d}\right)}, z_{l_{d}}^{-1}\right\rangle$.

- If $d=1$ and $h<l_{d}$, we have (by the first point of the proposition) $x_{h}^{\left(l_{d}\right)}=z_{h}=0$. So, in this case, each $P_{l} \in k\left\langle z_{l_{d}}^{-1}\right\rangle$ and

$$
x_{i}^{(j)}=x_{i}^{\left(l_{d}\right)}+Q_{1} z_{l_{d}}^{-1}+\cdots+Q_{K} z_{l_{d}}^{-K}
$$

with $K \geq 1$ and each $Q_{l}$ in $k$.

- Now, assume that $d>1$.

If $l_{d-1}<h \leq l_{d}-1$, we have (by (2)(a)) $x_{h}^{\left(l_{d}\right)}=z_{h}=0$ (since $h \in \Delta)$. So,

- If $i \geq l_{d-1}$, each $P_{l} \in k\left\langle z_{l_{d}}^{-1}\right\rangle$ and

$$
x_{i}^{(j)}=x_{i}^{\left(l_{d}\right)}+Q_{1} z_{l_{d}}^{-1}+\cdots+Q_{K} z_{l_{d}}^{-K}
$$

with $K \geq 1$ and each $Q_{l}$ in $k$.

- If $i<l_{d-1}$, each $P_{l} \in k\left\langle x_{i+1}^{\left(l_{d}\right)}, \ldots, x_{l_{d-1}}^{\left(l_{d}\right)}, z_{l_{d}}^{-1}\right\rangle$. As, by lemma 4.3.1, $z_{l_{d}}=x_{l_{d}}^{\left(l_{d}\right)} q$-commutes with $x_{i+1}^{\left(l_{d}\right)}, \ldots, x_{l_{d-1}}^{\left(l_{d}\right)}$, each $P_{l}$ can be written as follows:

$$
P_{l}=S_{0, l}+S_{1, l} z_{l_{d}}^{-1}+\cdots+S_{E, l} z_{l_{d}}^{-E}
$$

with $E \geq 1$ and each $S_{j, l} \in k\left\langle x_{i+1}^{\left(l_{d}\right)}, \ldots, x_{l_{d-1}}^{\left(l_{d}\right)}\right\rangle$. So, we conclude that, in this case,

$$
x_{i}^{(j)}=x_{i}^{\left(l_{d}\right)}+Q_{1} z_{l_{d}}^{-1}+\cdots+Q_{K} z_{l_{d}}^{-K}
$$

with $K \geq 1$ and each $Q_{l}$ in $k\left\langle x_{i+1}^{\left(l_{d}\right)}, \ldots, x_{l_{d-1}}^{\left(l_{d}\right)}\right\rangle$.

Proposition 4.3.2. Consider some $j$ in $\llbracket 2, \ldots, m \rrbracket$, assume that $i+1<j$ and consider some $u \in k\left\langle x_{i+1}^{(j)}, \ldots, x_{j-1}^{(j)}\right\rangle$.

(1) If $j \leq l_{1}$, then $u \in k$.

Assume that $l_{1}<j$ and denote by $d$ the greatest integer such that $l_{d}<j$ $(1 \leq d \leq p)$.

(2) (a) If $l_{d} \leq i$, then $u \in k$.

(b) If $i<l_{d}$, then

$$
u=u_{1} z_{l_{d}}^{a_{1}}+\cdots+u_{K} z_{l_{d}}^{a_{K}}
$$

with $K \geq 1,\left(a_{1}, \ldots, a_{K}\right) \in \mathbb{Z}^{K}$ and:

- If $d=1$, then each $u_{l} \in k$.

- If $d>1$ and $i \geq l_{d-1}$, then each $u_{l} \in k$.

- If $d>1$ and $i<l_{d-1}$, then each $u_{l} \in k\left\langle x_{i+1}^{\left(l_{d}\right)}, \ldots, x_{l_{d-1}}^{\left(l_{d}\right)}\right\rangle$.

Proof. (1) By (proposition 4.3.1, (1)) we have $x_{h}^{(j)}=0$ for any $h<j$. So, $u \in k$.

(2) (a) Consider some integer $h$ such that $l_{d}<h<j$. By (proposition 4.3.1, (2)(a)) we have $x_{h}^{(j)}=z_{h}=0($ since $h \in \Delta)$. So, $u \in k$. 
(b) Consider some integer $h$ with $i<h<j$.

If $l_{d}<h$, then, as in (2)(a), we have $x_{h}^{(j)}=0$. If $h=l_{d}$, then, again by (proposition 4.3.1, (2)(a)), we have $x_{h}^{(j)}=z_{h}=z_{l_{d}}$.

- Assume that $d=1$. If $h<l_{d}$, then, by (proposition 4.3.1, (2)(b)), we have

$$
x_{h}^{(j)}=x_{h}^{\left(l_{d}\right)}+Q_{1} z_{l_{d}}^{-1}+\cdots+Q_{M} z_{l_{d}}^{-M}
$$

with $M \geq 1$ and each $Q_{l}$ in $k$. By (proposition 4.3.1, (1)), we also have $x_{h}^{\left(l_{d}\right)}=0$. So, $x_{h}^{(j)} \in k\left\langle z_{l_{d}}^{-1}\right\rangle$ and it turns out that $u \in k\left\langle z_{l_{d}}^{ \pm 1}\right\rangle$. This implies that

$$
u=u_{1} z_{l_{d}}^{a_{1}}+\cdots+u_{K} z_{l_{d}}^{a_{K}}
$$

with $N \geq 1, \quad\left(a_{1}, \ldots, a_{N}\right) \in \mathbb{Z}^{N}$ and each $u_{l} \in k$.

- Assume that $d>1$ and $i \geq l_{d-1}$. If $i<h<l_{d}$, we have $l_{d-1}$ $<h<l_{d}$ and, by (proposition 4.3.1, (2)(b)),

$$
x_{h}^{(j)}=x_{h}^{\left(l_{d}\right)}+Q_{1} z_{l_{d}}^{-1}+\cdots+Q_{M} z_{l_{d}}^{-M}
$$

with $M \geq 1$ and each $Q_{l} \in k$. By (proposition 4.3.1, (1)) we also have $x_{h}^{\left(l_{d}\right)}=z_{h}$ and, since $h \in \Delta, x_{h}^{\left(l_{d}\right)}=0$. As above, we conclude that

$$
u=u_{1} z_{l_{d}}^{a_{1}}+\cdots+u_{K} z_{l_{d}}^{a_{K}}
$$

with $K \geq 1, \quad\left(a_{1}, \ldots, a_{K}\right) \in \mathbb{Z}^{K}$ and each $u_{l} \in k$.

- Assume that $d>1$ and $i<l_{d-1}$. If $i<h<l_{d}$, we have, always by (proposition 4.3.1, (2)(b)),

$$
x_{h}^{(j)}=x_{h}^{\left(l_{d}\right)}+Q_{1} z_{l_{d}}^{-1}+\cdots+Q_{M} z_{l_{d}}^{-M}
$$

with $M \geq 1$ and:

- If $l_{d-1}<h$, then each $Q_{l} \in k$ and, as in the previous case, $x_{h}^{\left(l_{d}\right)}=0$. So, $x_{h}^{(j)} \in k\left\langle z_{l_{d}}^{-1}\right\rangle$.

- If $h=l_{d-1}$, then each $Q_{l} \in k$ and, consequently, $x_{h}^{(j)} \in$ $k\left\langle x_{h}^{\left(l_{d}\right)}, z_{l_{d}}^{-1}\right\rangle=k\left\langle x_{l_{d-1}}^{\left(l_{d}\right)}, z_{l_{d}}^{-1}\right\rangle$.

- If $h<l_{d-1}$, then each $Q_{l} \in<x_{h+1}^{\left(l_{d}\right)}, \ldots, x_{l_{d-1}}^{\left(l_{d}\right)}>$ and, consequently, $x_{h}^{(j)} \in k\left\langle x_{h}^{\left(l_{d}\right)}, x_{h+1}^{\left(l_{d}\right)}, \ldots, x_{l_{d-1}}^{\left(l_{d}\right)}, z_{l_{d}}^{-1}\right\rangle$.

So, it turns out that $u \in k\left\langle x_{i+1}^{\left(l_{d}\right)}, \ldots, x_{l_{d-1}}^{\left(l_{d}\right)}, z_{l_{d}}^{ \pm 1}\right\rangle$. As, by lemma 4.3.1, $z_{l_{d}}=x_{l_{d}}^{\left(l_{d}\right)} q$-commutes with $x_{i+1}^{\left(l_{d}\right)}, \ldots, x_{l_{d-1}}^{\left(l_{d}\right)}$, we conclude that

$$
u=u_{1} z_{l_{d}}^{a_{1}}+\cdots+u_{K} z_{l_{d}}^{a_{K}}
$$

with $K \geq 1,\left(a_{1}, \ldots, a_{K}\right) \in \mathbb{Z}^{K}$ and each $u_{l}$ in $k\left\langle x_{i+1}^{\left(l_{d}\right)}, \ldots\right.$, $\left.x_{l_{d-1}}^{\left(l_{d}\right)}\right\rangle$.

Proposition 4.3.3. Consider some $j$ in $\llbracket 2, \ldots, m \rrbracket$ with $i<j$.

(1) If $\left\{l_{1}, \ldots, l_{p}\right\} \cap \llbracket i+1, \ldots, j-1 \rrbracket=\emptyset$, then $x_{i}^{(j)}=z_{i}$. 
(2) Assume that $\left\{l_{1}, \ldots, l_{p}\right\} \cap \llbracket i+1, \ldots, j-1 \rrbracket \neq \emptyset$ and set $\left\{l_{1}, \ldots, l_{p}\right\} \cap \llbracket i+1$, $\ldots, j-1 \rrbracket=\left\{l_{c}<\cdots<l_{d}\right\}$. Then, we have

$$
x_{i}^{(j)}=z_{i}+\sum_{\underline{a}=\left(a_{c}, \ldots, a_{d}\right) \in F} \eta(\underline{a}) z_{l_{c}}^{a_{c}} \ldots z_{l_{d}}^{a_{d}},
$$

where

- $F$ is a finite (possibly empty) subset of $\mathbb{Z}^{d-c+1}$.

- If $\preceq$ denotes the inverse lexicographic order in $\mathbb{Z}^{d-c+1}$, then, for any $\underline{a}$ in $F$, we have $\underline{a} \prec 0$ (i.e. $\underline{a}=\left(a_{c}, \ldots, a_{r}, 0, \ldots, 0\right)$ with $\left.a_{r}<0\right)$.

- For each $\underline{a}$ in $F$, we have $\eta(\underline{a}) \in k^{*}$.

Proof. We prove the proposition by induction on $j-i$. In order to do this, we first prove assertion (1).

So, assume that $\left\{l_{1}, \ldots, l_{p}\right\} \cap \llbracket i+1, \ldots, j-1 \rrbracket=\emptyset$, and observe that there are two possibilities:

(1) (a) $j \leq l_{1}$. By proposition 4.3.1, we know that $x_{i}^{(j)}=z_{i}$.

(b) $l_{1}<j$ and, if $d$ is the greatest integer such that $l_{d}<j, l_{d} \leq i<j$. Again, by proposition 4.3.1, we know that $x_{i}^{(j)}=z_{i}$.

Assume $j-i=1$. In this case, we have $\left\{l_{1}, \ldots, l_{p}\right\} \cap \llbracket i+1, \ldots, j-1 \rrbracket=$ $\emptyset$. So, we are in the case of assertion (1), and the proof is over.

Now assume $j-i>1$. As assertion (1) is already proved, we may assume that $\left\{l_{1}, \ldots, l_{p}\right\} \cap \llbracket i+1, \ldots, j-1 \rrbracket=\left\{l_{c}<\cdots<l_{d}\right\}$ is nonempty. So, we have $l_{1}<j, d$ is the greatest integer such that $l_{d}<j$ and $i<l_{d}<j$. Now, by proposition 4.3.1, we can write

$$
x_{i}^{(j)}=x_{i}^{\left(l_{d}\right)}+Q_{1} z_{l_{d}}^{-1}+\cdots+Q_{K} z_{l_{d}}^{-K}
$$

with $K \geq 1$ and three possible cases:

(1) $d=1$. Then each $Q_{l} \in k$ and, since $x_{i}^{\left(l_{d}\right)}=z_{i}$ (see proposition 4.3.1, (1)), the proof is over.

(2) $d>1$ and $i \geq l_{d-1}$. Then each $Q_{l} \in k$ and, since $x_{i}^{\left(l_{d}\right)}=z_{i}$ (see proposition 4.3.1, (2)(a)), the proof is over.

(3) $d>1$ and $i<l_{d-1}$ (so that $c \leq d-1$ ). In this case, each $Q_{l} \in k\left\langle x_{i+1}^{\left(l_{d}\right)}, \ldots\right.$, $\left.x_{l_{d-1}}^{\left(l_{d}\right)}\right\rangle$. Since $l_{d}<j$, it follows from the induction assumption that the proposition is true for each $x_{h}^{\left(l_{d}\right)}$ with $i \leq h<l_{d}$. This implies that

- If $i+1 \leq h \leq l_{d-1}$, we have $x_{h}^{\left(l_{d}\right)} \in k\left\langle z_{l_{c}}^{ \pm 1}, \ldots, z_{l_{d-1}}^{ \pm 1}\right\rangle$ (since $\left\{l_{1}, \ldots, l_{p}\right\} \cap$ $\llbracket h+1, \ldots, l_{d}-1 \rrbracket \subseteq\left\{l_{c}<\cdots<l_{d-1}\right\}$ and $z_{h}$ is either 0 or equal to $z_{l_{r}}$ with $\left.c \leq r \leq d-1\right)$. So, each $Q_{l} \in k\left\langle z_{l_{c}}^{ \pm 1}, \ldots, z_{l_{d-1}}^{ \pm 1}\right\rangle$.

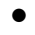

$$
x_{i}^{\left(l_{d}\right)}=z_{i}+\sum_{\underline{a}=\left(a_{c}, \ldots, a_{d-1}\right) \in F_{1}} \eta(\underline{a}) z_{l_{c}}^{a_{c}} \ldots z_{l_{d-1}}^{a_{d-1}},
$$

where

$\diamond F_{1}$ is a finite (possibly empty) subset of $\mathbb{Z}^{d-c}$.

$\diamond$ For each $\underline{a}$ in $F_{1}$, we have $\underline{a} \prec 0$ and $\eta(\underline{a}) \in k^{*}$.

As each $Q_{l}$ is a summand of (ordered) Laurent monomials in $z_{l_{c}}, \ldots, z_{l_{d-1}}$, it turns out that

$$
x_{i}^{(j)}=z_{i}+\sum_{\underline{a}=\left(a_{c}, \ldots, a_{d}\right) \in F} \eta(\underline{a}) z_{l_{c}}^{a_{c}} \ldots z_{l_{d}}^{a_{d}},
$$


where

$\diamond F$ is a finite (possibly empty) subset of $\mathbb{Z}^{d-c+1}$.

$\diamond$ For each $\underline{a}$ in $F$, we have $\underline{a} \prec 0$ and $\eta(\underline{a}) \in k^{*}$.

This proposition implies immediately

Corollary 4.3.1. Consider some integer $d$ with $1<d \leq p$. If $1 \leq i<l_{d}$, then $x_{i}^{\left(l_{d}\right)} \in k\left\langle z_{l_{1}}^{ \pm 1}, \ldots, z_{l_{d-1}}^{ \pm 1}\right\rangle$.

Proof. By proposition 4.3.3, $x_{i}^{\left(l_{d}\right)}=z_{i}+Q$ with $Q$ a (possibly 0) Laurent polynomial in $z_{l_{1}}, \ldots, z_{l_{d-1}}$. As $z_{i}$ is either 0 or $z_{l_{r}}$ with $1 \leq r \leq d-1$, the proof is over.

Corollary 4.3.2. Consider some integer $d$ with $1<d \leq p$. In $D_{m}$ considered as a left module over $k\left\langle x_{1}^{\left(l_{d}\right)}, \ldots, x_{l_{d}-1}^{\left(l_{d}\right)}\right\rangle$, the ordered Laurent monomials $z_{l_{d}}^{a_{d}} \ldots z_{l_{p}}^{a_{p}}\left(a_{d}, \ldots, a_{p}\right.$ in $\left.\mathbb{Z}\right)$ are linearly independent.

Proof. By corollary 4.3.1, we have $k\left\langle x_{1}^{\left(l_{d}\right)}, \ldots, x_{l_{d}-1}^{\left(l_{d}\right)}\right\rangle \subseteq k\left\langle z_{l_{1}}^{ \pm 1}, \ldots, z_{l_{d-1}}^{ \pm 1}\right\rangle$. So, the corollary results immediately from the $k$-linear independence of the ordered Laurent monomials in $z_{l_{1}}, \ldots, z_{l_{e}}$ (see proposition 4.2.2).

4.4. A new sufficient condition for $\mathcal{P}^{(m)}$ to be in $\operatorname{Im}\left(\phi_{m}\right)$. The notations are the same as in the preceding sections, but we do not assume that $\bar{\Delta}$ is nonempty a priori.

Assume that $\mathcal{P}^{(m)}$ is not in $\operatorname{Im}\left(\phi_{m}\right)$ so that, by proposition $4.1 .1, X_{m}^{(m)} \in \mathcal{P}^{(m)}$ and there is some $j$ in $\llbracket 1, \ldots, m-1 \rrbracket$ such that

$$
U=\Theta^{(m)}\left(\delta_{m}^{(m+1)}\left(X_{j}^{(m+1)}\right)\right) \notin \mathcal{P}^{(m)} .
$$

Let us choose such a $j$ maximal. So, we have

$$
\Theta^{(m)}\left(\delta_{m}^{(m+1)}\left(X_{i}^{(m+1)}\right)\right) \in \mathcal{P}^{(m)}
$$

for any $i$ in $\llbracket j+1, \ldots, m-1 \rrbracket$ and we observe that, since $U \notin \mathcal{P}^{(m)}$, we have $u:=$ $f_{m}(U) \neq 0$ in $A^{(m)}$.

Recall that

$$
\begin{gathered}
\Delta=\left\{i \in \llbracket 1, \ldots, t \rrbracket \mid Z_{i} \in \mathcal{P}^{(2)}\right\}=\left\{j_{1}<\cdots<j_{s}\right\} \text { (unless this set is empty), } \\
\bar{\Delta}=\| 1, \ldots, t \rrbracket \backslash \Delta=\left\{l_{1}<\cdots<l_{e}\right\} \text { (unless this set is empty). }
\end{gathered}
$$

Lemma 4.4.1. $\bar{\Delta}$ is nonempty, $l_{1}<m, m \notin \bar{\Delta}$ and, if $p$ denotes the greatest integer such that $l_{p}<m$, we have $j<l_{p}<m$.

Proof. First, recall that:

- If $\bar{\Delta}$ is empty, then (observation 4.2.1) each $x_{l}^{(m)}(l \in \llbracket 1, \ldots, t \rrbracket)$ is zero.

- $\delta_{m}^{(m+1)}\left(X_{j}^{(m+1)}\right)=P_{m, j}^{(m+1)}$. This is 0 if $m=j+1$ or a (finite) summand

$$
\sum_{\underline{a}=\left(a_{j+1}, \ldots, a_{m-1}\right)} c_{\underline{a}}\left(X_{j+1}^{(m+1)}\right)^{a_{j+1}} \ldots\left(X_{m-1}^{(m+1)}\right)^{a_{m-1}}
$$

with each $\underline{a}$ in $\mathbb{N}^{m-1-j}$ if $m>j+1$ and, moreover, $\underline{a}$ is nonzero when $c_{a}$ $\neq 0$.

- $\Theta^{(m)}: k\left\langle X_{1}^{(m+1)}, \ldots, X_{m-1}^{(m+1)}\right\rangle \rightarrow k\left\langle X_{1}^{(m)}, \ldots, X_{m-1}^{(m)}\right\rangle$ is an algebra homomorphism which transforms each $X_{l}^{(m+1)}$ into $X_{l}^{(m)}$. 
Assume that $m=j+1$. In this case, we have

$$
\delta_{m}^{(m+1)}\left(X_{j}^{(m+1)}\right)=0 \Rightarrow U=\Theta^{(m)}\left(\delta_{m}^{(m+1)}\left(X_{j}^{(m+1)}\right)\right)=0 \Rightarrow u=f_{m}(U)=0
$$

and we obtain a contradiction.

So, we have $m>j+1$, and

$$
\begin{aligned}
\delta_{m}^{(m+1)}\left(X_{j}^{(m+1)}\right) & =\sum_{\underline{a}=\left(a_{j+1}, \ldots, a_{m-1}\right)} c_{\underline{a}}\left(X_{j+1}^{(m+1)}\right)^{a_{j+1}} \ldots\left(X_{m-1}^{(m+1)}\right)^{a_{m-1}} \\
& \Rightarrow U=\Theta^{(m)}\left(\Delta_{m}^{(m+1)}\left(X_{j}^{(m+1)}\right)\right) \\
& =\sum_{\underline{a}=\left(a_{j+1}, \ldots, a_{m-1}\right)} c_{\underline{a}}\left(X_{j+1}^{(m)}\right)^{a_{j+1}} \ldots\left(X_{m-1}^{(m)}\right)^{a_{m-1}} \\
& \Rightarrow=f_{m}(U)=\sum_{\underline{a}=\left(a_{j+1}, \ldots, a_{m-1}\right)} c_{\underline{a}}\left(x_{j+1}^{(m)}\right)^{a_{j+1}} \ldots\left(x_{m-1}^{(m)}\right)^{a_{m-1}} .
\end{aligned}
$$

Assume that $\bar{\Delta}$ is empty. Then, each $x_{l}^{(m)}$ is zero and, since $\underline{a}$ is nonzero when $c_{\underline{a}} \neq 0$, we get that $u=0$ and we still have a contradiction. So, $\bar{\Delta}$ is nonempty.

If $l_{1} \geq m$, then, for each $l \in \llbracket 1, \ldots, m-1 \rrbracket$, we have (by lemma 4.3 .2 and, since $\left.l<l_{1}\right) x_{l}^{(m)}=z_{l}=0$. As above, this implies that $u=0$ and we still get a contradiction. So, $l_{1}<m$.

Since $X_{m}^{(m)} \in \mathcal{P}^{(m)}$, we have $x_{m}^{(m)}=0 . \quad$ As $x_{m}^{(m)}=z_{m}$ (lemma 4.3.1), we have $Z_{m} \in \mathcal{P}^{(2)}$ and so, $m \in \Delta$. Moreover, if $l_{p} \leq j$, then, for each $l \in \llbracket j+1, \ldots, m-1 \rrbracket$, we have (by propostion 4.3.1, (2)(a) and, since $\left.l_{p}<l<m\right) x_{l}^{(m)}=z_{l}=0$. As above, this implies that $u=0$ and we still get a contradiction. So, $j<l_{p}$.

Now, set $V=\delta_{m}^{(m+1)}\left(X_{j}^{(m+1)}\right)=X_{m}^{(m+1)} X_{j}^{(m+1)}-q^{-\left(\beta_{m}, \beta_{j}\right)} X_{j}^{(m+1)} X_{m}^{(m+1)}$ (see section 3.2), so that $U=\Theta^{(m)}(V)$.

Lemma 4.4.2. Consider some integer $l$ with $j<l<m$. Then we have, in $R^{(m+1)}$,

$$
\begin{gathered}
X_{l}^{(m+1)} V=q^{\left(\beta_{l}, \beta_{m}-\beta_{j}\right)} V X_{l}^{(m+1)} \\
+q^{\left(\beta_{l}, \beta_{m}\right)}\left[q^{-\left(\beta_{j}, \beta_{l}+\beta_{m}\right)} X_{j}^{(m+1)} \delta_{m}^{(m+1)}\left(X_{l}^{(m+1)}\right)-\delta_{m}^{(m+1)}\left(X_{l}^{(m+1)}\right) X_{j}^{(m+1)}\right] \\
+q^{\left(\beta_{l}, \beta_{m}\right)} \delta_{m}^{(m+1)} \circ \delta_{l}^{(m+1)}\left(X_{j}^{(m+1)}\right) .
\end{gathered}
$$

Proof. We observe that, as each $X_{i}^{(m+1)}$ is homogeneous of degree $\beta_{i}$ (see section 3.2 ), we have

$$
\begin{gathered}
\delta_{l}^{(m+1)}(V)=\delta_{l}^{(m+1)}\left(X_{m}^{(m+1)}\right) X_{j}^{(m+1)}+q^{-\left(\beta_{l}, \beta_{m}\right)} X_{m}^{(m+1)} \delta_{l}^{(m+1)}\left(X_{j}^{(m+1)}\right) \\
-q^{-\left(\beta_{m}, \beta_{j}\right)} \delta_{l}^{(m+1)}\left(X_{j}^{(m+1)}\right) X_{m}^{(m+1)}-q^{-\left[\left(\beta_{m}, \beta_{j}\right)+\left(\beta_{l}, \beta_{j}\right)\right]} X_{j}^{(m+1)} \delta_{l}^{(m+1)}\left(X_{m}^{(m+1)}\right) .
\end{gathered}
$$

So, we can write

with

$$
\delta_{l}^{(m+1)}(V)=A+B-C-D
$$

$$
\begin{gathered}
A=\delta_{l}^{(m+1)}\left(X_{m}^{(m+1)}\right) X_{j}^{(m+1)}, \\
B=q^{-\left(\beta_{l}, \beta_{m}\right)} X_{m}^{(m+1)} \delta_{l}^{(m+1)}\left(X_{j}^{(m+1)}\right), \\
C=q^{-\left(\beta_{m}, \beta_{j}\right)} \delta_{l}^{(m+1)}\left(X_{j}^{(m+1)}\right) X_{m}^{(m+1)}, \\
D=q^{-\left[\left(\beta_{m}, \beta_{j}\right)+\left(\beta_{l}, \beta_{j}\right)\right]} X_{j}^{(m+1)} \delta_{l}^{(m+1)}\left(X_{m}^{(m+1)}\right) .
\end{gathered}
$$


Now, we compute separately the different pieces of the right member:

- $\delta_{l}^{(m+1)}\left(X_{m}^{(m+1)}\right)=X_{l}^{(m+1)} X_{m}^{(m+1)}-q^{-\left(\beta_{l}, \beta_{m}\right)} X_{m}^{(m+1)} X_{l}^{(m+1)}$. As we also have $\delta_{m}^{(m+1)}\left(X_{l}^{(m+1)}\right)=X_{m}^{(m+1)} X_{l}^{(m+1)}-q^{-\left(\beta_{l}, \beta_{m}\right)} X_{l}^{(m+1)} X_{m}^{(m+1)}$, we can write $X_{l}^{(m+1)} X_{m}^{(m+1)}=q^{\left(\beta_{l}, \beta_{m}\right)}\left[X_{m}^{(m+1)} X_{l}^{(m+1)}-\delta_{m}^{(m+1)}\left(X_{l}^{(m+1)}\right)\right]$.

So, we get:

$\delta_{l}^{(m+1)}\left(X_{m}^{(m+1)}\right)=\left(q^{\left(\beta_{l}, \beta_{m}\right)}-q^{-\left(\beta_{l}, \beta_{m}\right)}\right) X_{m}^{(m+1)} X_{l}^{(m+1)}-q^{\left(\beta_{l}, \beta_{m}\right)} \delta_{m}^{(m+1)}\left(X_{l}^{(m+1)}\right)$.

- From this, we deduce that

$$
\begin{aligned}
& \delta_{l}^{(m+1)}\left(X_{m}^{(m+1)}\right) X_{j}^{(m+1)}=\left(q^{\left(\beta_{l}, \beta_{m}\right)}-q^{-\left(\beta_{l}, \beta_{m}\right)}\right) X_{m}^{(m+1)} X_{l}^{(m+1)} X_{j}^{(m+1)} \\
& \quad-q^{\left(\beta_{l}, \beta_{m}\right)} \delta_{m}^{(m+1)}\left(X_{l}^{(m+1)}\right) X_{j}^{(m+1)} .
\end{aligned}
$$

But we also have

$$
\delta_{l}^{(m+1)}\left(X_{j}^{(m+1)}\right)=X_{l}^{(m+1)} X_{j}^{(m+1)}-q^{-\left(\beta_{l}, \beta_{j}\right)} X_{j}^{(m+1)} X_{l}^{(m+1)},
$$

so that

$$
\begin{aligned}
\delta_{l}^{(m+1)} & \left(X_{m}^{(m+1)}\right) X_{j}^{(m+1)} \\
= & \left(q^{\left(\beta_{l}, \beta_{m}\right)}-q^{-\left(\beta_{l}, \beta_{m}\right)}\right) X_{m}^{(m+1)}\left[q^{-\left(\beta_{l}, \beta_{j}\right)} X_{j}^{(m+1)} X_{l}^{(m+1)}+\delta_{l}^{(m+1)}\left(X_{j}^{(m+1)}\right)\right] \\
& -q^{\left(\beta_{l}, \beta_{m}\right)} \delta_{m}^{(m+1)}\left(X_{l}^{(m+1)}\right) X_{j}^{(m+1)} .
\end{aligned}
$$

So, we get:

$$
\begin{gathered}
A=\left(q^{\left(\beta_{l}, \beta_{m}\right)}-q^{-\left(\beta_{l}, \beta_{m}\right)}\right) q^{-\left(\beta_{l}, \beta_{j}\right)} X_{m}^{(m+1)} X_{j}^{(m+1)} X_{l}^{(m+1)} \\
+\left(q^{\left(\beta_{l}, \beta_{m}\right)}-q^{-\left(\beta_{l}, \beta_{m}\right)}\right) X_{m}^{(m+1)} \delta_{l}^{(m+1)}\left(X_{j}^{(m+1)}\right) \\
-q^{\left(\beta_{l}, \beta_{m}\right)} \delta_{m}^{(m+1)}\left(X_{l}^{(m+1)}\right) X_{j}^{(m+1)}
\end{gathered}
$$

- Now, we have

$$
\begin{aligned}
A+B-C= & \left(q^{\left(\beta_{l}, \beta_{m}\right)}-q^{-\left(\beta_{l}, \beta_{m}\right)}\right) q^{-\left(\beta_{l}, \beta_{j}\right)} X_{m}^{(m+1)} X_{j}^{(m+1)} X_{l}^{(m+1)} \\
+ & q^{\left(\beta_{l}, \beta_{m}\right)} X_{m}^{(m+1)} \delta_{l}^{(m+1)}\left(X_{j}^{(m+1)}\right)-q^{-\left(\beta_{m}, \beta_{j}\right)} \delta_{l}^{(m+1)}\left(X_{j}^{(m+1)}\right) X_{m}^{(m+1)} \\
& -q^{\left(\beta_{l}, \beta_{m}\right)} \delta_{m}^{(m+1)}\left(X_{l}^{(m+1)}\right) X_{j}^{(m+1)} \\
= & \left(q^{\left(\beta_{l}, \beta_{m}\right)}-q^{-\left(\beta_{l}, \beta_{m}\right)}\right) q^{-\left(\beta_{l}, \beta_{j}\right)} X_{m}^{(m+1)} X_{j}^{(m+1)} X_{l}^{(m+1)} \\
& +q^{\left(\beta_{l}, \beta_{m}\right)}\left[X_{m}^{(m+1)} \delta_{l}^{(m+1)}\left(X_{j}^{(m+1)}\right)\right. \\
& \left.-q^{-\left(\beta_{m}, \beta_{l}+\beta_{j}\right)} \delta_{l}^{(m+1)}\left(X_{j}^{(m+1)}\right) X_{m}^{(m+1)}\right] \\
& -q^{\left(\beta_{l}, \beta_{m}\right)} \delta_{m}^{(m+1)}\left(X_{l}^{(m+1)}\right) X_{j}^{(m+1)} .
\end{aligned}
$$

Since $\delta_{l}^{(m+1)}\left(X_{j}^{(m+1)}\right)$ is homogeneous of degree $\beta_{l}+\beta_{j}$, we have

$$
\begin{aligned}
& X_{m}^{(m+1)} \delta_{l}^{(m+1)}\left(X_{j}^{(m+1)}\right)-q^{-\left(\beta_{m}, \beta_{l}+\beta_{j}\right)} \delta_{l}^{(m+1)}\left(X_{j}^{(m+1)}\right) X_{m}^{(m+1)} \\
& \quad=\delta_{m}^{(m+1)} \circ \delta_{l}^{(m+1)}\left(X_{j}^{(m+1)}\right) .
\end{aligned}
$$

So, we get:

$$
\begin{gathered}
A+B-C \\
=\left(q^{\left(\beta_{l}, \beta_{m}\right)}-q^{-\left(\beta_{l}, \beta_{m}\right)}\right) q^{-\left(\beta_{l}, \beta_{j}\right)} X_{m}^{(m+1)} X_{j}^{(m+1)} X_{l}^{(m+1)} \\
+q^{\left(\beta_{l}, \beta_{m}\right)} \delta_{m}^{(m+1)} \circ \delta_{l}^{(m+1)}\left(X_{j}^{(m+1)}\right)-q^{\left(\beta_{l}, \beta_{m}\right)} \delta_{m}^{(m+1)}\left(X_{l}^{(m+1)}\right) X_{j}^{(m+1)} .
\end{gathered}
$$


- Using the computation of $\delta_{l}^{(m+1)}\left(X_{m}^{(m+1)}\right)$ made in the first point above, we can write:

$$
\begin{aligned}
D & =q^{-\left[\left(\beta_{m}, \beta_{j}\right)+\left(\beta_{l}, \beta_{j}\right)\right]} X_{j}^{(m+1)} \delta_{l}^{(m+1)}\left(X_{m}^{(m+1)}\right) \\
& =q^{-\left(\beta_{j}, \beta_{l}+\beta_{m}\right)} X_{j}^{(m+1)}\left[\left(q^{\left(\beta_{l}, \beta_{m}\right)}-q^{-\left(\beta_{l}, \beta_{m}\right)}\right) X_{m}^{(m+1)} X_{l}^{(m+1)}-q^{\left(\beta_{l}, \beta_{m}\right)} \delta_{m}^{(m+1)}\left(X_{l}^{(m+1)}\right)\right] .
\end{aligned}
$$

So, we get:

$$
\begin{gathered}
D=q^{-\left(\beta_{j}, \beta_{l}+\beta_{m}\right)}\left(q^{\left(\beta_{l}, \beta_{m}\right)}-q^{-\left(\beta_{l}, \beta_{m}\right)}\right) X_{j}^{(m+1)} X_{m}^{(m+1)} X_{l}^{(m+1)} \\
-q^{-\left(\beta_{j}, \beta_{l}+\beta_{m}\right)} q^{\left(\beta_{l}, \beta_{m}\right)} X_{j}^{(m+1)} \delta_{m}^{(m+1)}\left(X_{l}^{(m+1)}\right) .
\end{gathered}
$$

- Now, we have

$$
\begin{aligned}
& \delta_{l}^{(m+1)}(V)=A+B-C-D \\
& \left.=q^{-\left(\beta_{j}, \beta_{l}\right.}\right)\left(q^{\left(\beta_{l}, \beta_{m}\right)}-q^{-\left(\beta_{l}, \beta_{m}\right)}\right)\left[X_{m}^{(m+1)} X_{j}^{(m+1)}-q^{-\left(\beta_{j}, \beta_{m}\right)} X_{j}^{(m+1)} X_{m}^{(m+1)}\right] X_{l}^{(m+1)} \\
& \quad+q^{\left(\beta_{l}, \beta_{m}\right)}\left[q^{-\left(\beta_{j}, \beta_{l}+\beta_{m}\right)} X_{j}^{(m+1)} \delta_{m}^{(m+1)}\left(X_{l}^{(m+1)}\right)-\delta_{m}^{(m+1)}\left(X_{l}^{(m+1)}\right) X_{j}^{(m+1)}\right] \\
& \quad+q^{\left(\beta_{l}, \beta_{m}\right)} \delta_{m}^{(m+1)} \circ \delta_{l}^{(m+1)}\left(X_{j}^{(m+1)}\right) .
\end{aligned}
$$

So, we get:

$$
\begin{gathered}
\left.\delta_{l}^{(m+1)}(V)=q^{-\left(\beta_{j}, \beta_{l}\right.}\right)\left(q^{\left(\beta_{l}, \beta_{m}\right)}-q^{-\left(\beta_{l}, \beta_{m}\right)}\right) V X_{l}^{(m+1)} \\
+q^{\left(\beta_{l}, \beta_{m}\right)}\left[q^{-\left(\beta_{j}, \beta_{l}+\beta_{m}\right)} X_{j}^{(m+1)} \delta_{m}^{(m+1)}\left(X_{l}^{(m+1)}\right)-\delta_{m}^{(m+1)}\left(X_{l}^{(m+1)}\right) X_{j}^{(m+1)}\right] \\
+q^{\left(\beta_{l}, \beta_{m}\right)} \delta_{m}^{(m+1)} \circ \delta_{l}^{(m+1)}\left(X_{j}^{(m+1)}\right) .
\end{gathered}
$$

As $V$ is homogeneous of degree $\beta_{j}+\beta_{m}$, we have also

$$
\delta_{l}^{(m+1)}(V)=X_{l}^{(m+1)} V-q^{-\left(\beta_{l}, \beta_{j}+\beta_{m}\right)} V X_{l}^{(m+1)},
$$

which gives the required formula.

Now, let us observe that, if $j<l<m$, we have the following results:

- $\delta_{m}^{(m+1)}\left(X_{l}^{(m+1)}\right) \in k\left\langle X_{l+1}^{(m+1)}, \ldots, X_{m-1}^{(m+1)}\right\rangle$ (or is zero if $l=m-1$ ) (see section 3.2).

- Similarly, $V=\delta_{m}^{(m+1)}\left(X_{j}^{(m+1)}\right) \in k\left\langle X_{j+1}^{(m+1)}, \ldots, X_{m-1}^{(m+1)}\right\rangle$ (observe that $j<m-1$ by lemma 4.4.1).

- $\delta_{l}^{(m+1)}\left(X_{j}^{(m+1)}\right) \in k\left\langle X_{j+1}^{(m+1)}, \ldots, X_{l-1}^{(m+1)}\right\rangle$ (or is zero if $l=j+1$ ), which implies that $\delta_{m}^{(m+1)} \circ \delta_{l}^{(m+1)}\left(X_{j}^{(m+1)}\right) \in k\left\langle X_{j+1}^{(m+1)}, \ldots, X_{m-1}^{(m+1)}\right\rangle$.

So, $V, \delta_{m}^{(m+1)}\left(X_{l}^{(m+1)}\right), \delta_{l}^{(m+1)} \circ \delta_{l}^{(m+1)}\left(X_{j}^{(m+1)}\right), X_{l}^{(m+1)}, X_{j}^{(m+1)}$ are all in $k\left\langle X_{1}^{(m+1)}, \ldots, X_{m-1}^{(m+1)}\right\rangle$ and, if we transform the equality of lemma 4.4 .2 by the algebra homomorphism $\Theta^{(m)}$, we obtain:

\section{Lemma 4.4.3.}

$$
\begin{gathered}
X_{l}^{(m)} U=q^{\left(\beta_{l}, \beta_{m}-\beta_{j}\right)} U X_{l}^{(m)} \\
+q^{\left(\beta_{l}, \beta_{m}\right)}\left[q^{-\left(\beta_{j}, \beta_{l}+\beta_{m}\right)} X_{j}^{(m)} \Theta^{(m)}\left(\delta_{m}^{(m+1)}\left(X_{l}^{(m+1)}\right)\right)-\Theta^{(m)}\left(\delta_{m}^{(m+1)}\left(X_{l}^{(m+1)}\right)\right) X_{j}^{(m)}\right] \\
+q^{\left(\beta_{l}, \beta_{m}\right)} \Theta^{(m)}\left(\delta_{m}^{(m+1)} \circ \delta_{l}^{(m+1)}\left(X_{j}^{(m+1)}\right)\right) .
\end{gathered}
$$


- It results from the choice of $j$ that $\Theta^{(m)}\left(\delta_{m}^{(m+1)}\left(X_{l}^{(m+1)}\right)\right) \in \mathcal{P}^{(m)}$.

Assume that $l>j+1$, so that (by section 3.2)

$$
\delta_{l}^{(m+1)}\left(X_{j}^{(m+1)}\right)=\sum_{\underline{a}=\left(a_{j+1}, \ldots, a_{l-1}\right)} c_{\underline{a}}\left(X_{j+1}^{(m+1)}\right)^{a_{j+1}} \ldots\left(X_{l-1}^{(m+1)}\right)^{a_{l-1}}
$$

with each $c_{a} \in k$.

As each $X_{i}^{(m+1)}$ is a $h_{m}$-eigenvector, each $\left.\delta_{m}^{(m+1)}\left(X_{j+1}^{(m+1)}\right)^{a_{j+1}} \ldots\left(X_{l-1}^{(m+1)}\right)^{a_{l-1}}\right)$ is a linear combination of products $M_{1} \delta_{m}^{(m+1)}\left(X_{i}^{(m+1)}\right) M_{2}$ with $j<i<l$ and $M_{1}$ (resp. $M_{2}$ ) an ordered monomial in $X_{j+1}^{(m+1)}, \ldots, X_{i}^{(m+1)}$ (resp. in $X_{i}^{(m+1)}, \ldots$, $\left.X_{l-1}^{(m+1)}\right)$. This implies that $\Theta^{(m)}\left(\delta_{m}^{(m+1)} \circ \delta_{l}^{(m+1)}\left(X_{j}^{(m+1)}\right)\right)$ is a linear combination of products $N_{1} \Theta^{(m)}\left(\delta_{m}^{(m+1)}\left(X_{i}^{(m+1)}\right)\right) N_{2}$ with $j<i<l, N_{1}$ (resp. $N_{2}$ ) an ordered monomial in $X_{j+1}^{(m)}, \ldots, X_{i}^{(m)}$ (resp. in $X_{i}^{(m)}, \ldots, X_{l-1}^{(m)}$ ).

As $\Theta^{(m)}\left(\delta_{m}^{(m+1)}\left(X_{i}^{(m+1)}\right)\right) \in \mathcal{P}^{(m)}$ for $j<i<m$, we conclude that

- $\Theta^{(m)}\left(\delta_{m}^{(m+1)} \circ \delta_{l}^{(m+1)}\left(X_{j}^{(m+1)}\right)\right) \in \mathcal{P}^{(m)}$.

We observe that this result is also true when $l=j+1$ since, in this case, $\delta_{l}^{(m+1)}\left(X_{j}^{(m+1)}\right)$ is zero (see section 3.2 ). So, if we transform the equality of lemma 4.4 .3 by the algebra homomorphism $f_{m}$, we obtain:

\section{Lemma 4.4.4.}

$$
x_{l}^{(m)} u=q^{\left(\beta_{l}, \beta_{m}-\beta_{j}\right)} u x_{l}^{(m)} .
$$

Recall (see section 3.2) that $V=\delta_{m}^{(m+1)}\left(X_{j}^{(m+1)}\right)$ ) is a homogeneous polynomial in $X_{j+1}^{(m+1)}, \ldots, X_{m-1}^{(m+1)}$ of degree $\beta_{m}+\beta_{j}$. So, since $\Theta^{(m)}$ transforms each $X_{i}^{(m+1)}$ $(1 \leq i<m)$ into $X_{i}^{(m)}, U=\Theta^{(m)}(V)$ is a homogeneous polynomial in $X_{j+1}^{(m)}$, $\ldots, X_{m-1}^{(m)}$ of degree $\beta_{m}+\beta_{j}$. This implies:

Lemma 4.4.5. $u$ is a nonzero polynomial in $x_{j+1}^{(m)}, \ldots, x_{m-1}^{(m)}$. Moreover, $u$ is homogeneous of degree $\beta_{m}+\beta_{j}$.

Proof. $u=f_{m}(U)$ is nonzero by construction. Since $f_{m}$ transforms each $X_{i}^{(m)}$ into $x_{i}^{(m)}$, it turns out that $u$ is a polynomial in $x_{j+1}^{(m)}, \ldots, x_{m-1}^{(m)}$. Moreover, $u=f_{m}(U)$ is homogeneous of degree $\beta_{m}+\beta_{j}$ by lemma 4.3.1.

Recall (lemma 4.4.1) that $j<l_{p}$ and that $p$ is the greatest integer such that $l_{p}<m$. Denote by $c$ the smallest integer such that $j<l_{c}(1 \leq c \leq p)$. As $u \in$ $k\left\langle x_{j+1}^{(m)}, \ldots, x_{m-1}^{(m)}\right\rangle$, it follows from (proposition 4.3.2, (2)(b)) that

$$
u=u_{1} z_{l_{p}}^{a_{1}}+\cdots+u_{M} z_{l_{p}}^{a_{M}}
$$

with $M \geq 1,\left(a_{1}, \ldots, a_{M}\right) \in \mathbb{Z}^{M}$ and:

- If $p=1$, then each $u_{i} \in k$.

- If $p>1$ and $j \geq l_{p-1}$, then each $u_{i} \in k$.

- If $p>1$ and $j<l_{p-1}$, then each $u_{i} \in k\left\langle x_{j+1}^{\left(l_{p}\right)}, \ldots, x_{l_{p-1}}^{\left(l_{p}\right)}\right\rangle$, so that we can write:

- If $c=p$, then each $u_{i} \in k$.

- If $c<p$, then each $u_{i} \in k\left\langle x_{j+1}^{\left(l_{p}\right)}, \ldots, x_{l_{p-1}}^{\left(l_{p}\right)}\right\rangle$.

In both cases, we may assume that $u_{1}, \ldots, u_{M}$ are all nonzero, that $a_{1}<\cdots<$ $a_{M}$, and we observe that 
- Each $u_{i} \in k\left\langle x_{1}^{\left(l_{p}\right)}, \ldots, x_{l_{p-1}}^{\left(l_{p}\right)}\right\rangle$ and is homogeneous of degree $\beta_{m}+\beta_{j}-a_{i} \beta_{l_{p}}$.

In fact, since $u$ is homogeneous of degree $\beta_{m}+\beta_{j}$ and $z_{l_{p}}=x_{l_{p}}^{\left(l_{p}\right)}$ is homogeneous of degree $\beta_{l_{p}}$, we have, for any $\rho \in \mathbb{Z} \Pi$,

$$
\begin{aligned}
& \overline{h_{\rho}}(u)=q^{-\left(\rho, a_{1} \beta_{l_{p}}\right)} \overline{h_{\rho}}\left(u_{1}\right) z_{l_{p}}^{a_{1}}+\cdots+q^{-\left(\rho, a_{M} \beta_{l_{p}}\right)} \overline{h_{\rho}}\left(u_{M}\right) z_{l_{p}}^{a_{M}} \\
& =q^{-\left(\rho, \beta_{m}+\beta_{j}\right)}\left[u_{1} z_{l_{p}}^{a_{1}}+\cdots+u_{M} z_{l_{p}}^{a_{M}}\right]
\end{aligned}
$$

and, by corollary 4.3.2, we can identify the coefficients of $z_{l_{p}}^{a_{i}}$, so that $q^{-\left(\rho, a_{i} \beta_{l_{p}}\right)} \overline{h_{\rho}}\left(u_{i}\right)$ $=q^{-\left(\rho, \beta_{m}+\beta_{j}\right)} u_{i}$ and $\overline{h_{\rho}}\left(u_{i}\right)=q^{-\left(\rho, \beta_{m}+\beta_{j}-a_{i} \beta_{l_{p}}\right)} u_{i}$.

By (proposition 4.3.3, (1)) we have $x_{l_{p}}^{(m)}=z_{l_{p}}=x_{l_{p}}^{\left(l_{p}\right)}$. So, using lemma 4.4.4 with $l=l_{p}$, we obtain:

$$
\left.z_{l_{p}} u=q^{\left(\beta_{l_{p}}, \beta_{m}-\beta_{j}\right)}\right) u z_{l_{p}}
$$

and, by (lemma 4.3.1, (4)),

$$
z_{l_{p}} u=\overline{h_{l_{p}}}\left(u_{1}\right) z_{l_{p}}^{a_{1}+1}+\cdots+\overline{h_{l_{p}}}\left(u_{M}\right) z_{l_{p}}^{a_{M}+1} .
$$

This implies that

$$
\begin{gathered}
q^{\left(\beta_{l_{p}}, \beta_{m}-\beta_{j}\right)}\left[u_{1} z_{l_{p}}^{a_{1}}+\cdots+u_{M} z_{l_{p}}^{a_{M}}\right] z_{l_{p}} \\
=\left[q^{-\left(\beta_{l_{p}}, \beta_{m}+\beta_{j}-a_{1} \beta_{l_{p}}\right)} u_{1} z_{l_{p}}^{a_{1}}+\cdots+q^{-\left(\beta_{l_{p}}, \beta_{m}+\beta_{j}-a_{N} \beta_{l_{p}}\right)} u_{M} z_{l_{p}}^{a_{M}}\right] z_{l_{p}} .
\end{gathered}
$$

As above, if $1 \leq i \leq M$, we can identify the coefficients of $z_{l_{p}}^{a_{i}+1}$, so that, since $u_{i} \neq 0$ and $q$ is not a root of unity, we have $-\left(\beta_{l_{p}}, \beta_{m}+\beta_{j}-a_{i} \beta_{l_{p}}\right)=\left(\beta_{l_{p}}, \beta_{m}-\beta_{j}\right)$. So, $a_{i}\left\|\beta_{l_{p}}\right\|^{2}=2\left(\beta_{l_{p}}, \beta_{m}\right)$ and then $a_{i}=\left(\beta_{l_{p}}^{\vee}, \beta_{m}\right)$.

This implies that $M=1$ and we conclude:

\section{Lemma 4.4.6.}

$$
u=u_{p} z_{l_{p}}^{a_{p}}
$$

with:

- If $c=p$, then $u_{p} \in k^{*}$.

- If $c<p$, then $u_{p} \in k\left\langle x_{j+1}^{\left(l_{p}\right)}, \ldots, x_{l_{p-1}}^{\left(l_{p}\right)}\right\rangle \backslash\{0\}$.

- $a_{p}=\left(\beta_{l_{p}}^{\vee}, \beta_{m}\right)$.

- $u_{p}$ is homogeneous of degree $\beta_{m}+\beta_{j}-a_{p} \beta_{l_{p}}=\gamma_{p}+\beta_{j}$ with $\gamma_{p}=s_{\beta_{l_{p}}}\left(\beta_{m}\right)$.

Now, we extend this result as follows:

Lemma 4.4 1. Consider some integer $d$ with $c \leq d \leq p$. Then

$$
u=u_{d} z_{l_{d}}^{a_{d}} \ldots z_{l_{p}}^{a_{p}}
$$

with:

- If $c=d$, then $u_{d} \in k^{*}$.

- If $c<d$, then $u_{d} \in k\left\langle x_{j+1}^{\left(l_{d}\right)}, \ldots, x_{l_{d-1}}^{\left(l_{d}\right)}\right\rangle \backslash\{0\}$.

- Denote by $\left(\gamma_{d}, \ldots, \gamma_{p}, \gamma_{p+1}\right)$ the sequence of (not necessarily positive) roots recursively defined by $\gamma_{p+1}=\beta_{m}$ and, for $d \leq i \leq p, \quad \gamma_{i}=s_{\beta_{l_{i}}}\left(\gamma_{i+1}\right)$. Then, each $a_{i}=\left(\beta_{l_{i}}^{\vee}, \gamma_{i+1}\right)$.

- $u_{d}$ is homogeneous of degree $\gamma_{d}+\beta_{j}$. 
Proof. We proceed by decreasing induction on $d$.

If $d=p$, everything has been done in lemma 4.4.6.

Assume that $c \leq d<p$ and that the lemma is true at the rank $d+1$, namely:

with:

$$
u=u_{d+1} z_{l_{d+1}}^{a_{d+1}} \ldots z_{l_{p}}^{a_{p}}
$$

- $u_{d+1} \in k\left\langle x_{j+1}^{\left(l_{d+1}\right)}, \ldots, x_{l_{d}}^{\left(l_{d+1}\right)}\right\rangle \backslash\{0\}$.

- If $\left(\gamma_{d+1}, \ldots, \gamma_{p}, \gamma_{p+1}\right)$ is the sequence of (not necessarily positive) roots recursively defined by $\gamma_{p+1}=\beta_{m}$ and, for $d+1 \leq i \leq p, \gamma_{i}=s_{\beta_{l_{i}}}\left(\gamma_{i+1}\right)$, then, each $a_{i}=\left(\beta_{l_{i}}^{\vee}, \gamma_{i+1}\right)$.

- $u_{d+1}$ is homogeneous of degree $\gamma_{d+1}+\beta_{j}$.

By (proposition 4.3.2, (2)(b)), we have:

$$
u_{d+1}=v_{1} z_{l_{d}}^{b_{1}}+\cdots+v_{M} z_{l_{d}}^{b_{M}}
$$

with $M \geq 1,\left(b_{1}, \ldots, b_{M}\right) \in \mathbb{Z}^{M}$ and:

- If $d=c$, then each $v_{i} \in k$.

- If $d>c$, then each $v_{i} \in k\left\langle x_{j+1}^{\left(l_{d}\right)}, \ldots, x_{l_{d-1}}^{\left(l_{d}\right)}\right\rangle$.

As above, we may assume that $v_{1}, \ldots, v_{M}$ are all nonzero, that $b_{1}<\cdots<b_{M}$, and observe that

- Each $v_{i}$ is homogeneous of degree $\gamma_{d+1}+\beta_{j}-b_{i} \beta_{l_{d}}$.

By (proposition 4.3.3, (2)), we have

$$
x_{l_{d}}^{(m)}=z_{l_{d}}+\sum_{\underline{s}=\left(s_{d+1}, \ldots, s_{p}\right) \in F} \eta(\underline{s}) z_{l_{d+1}}^{s_{d+1}} \ldots z_{l_{p}}^{s_{p}}
$$

where:

- $F$ is a finite (possibly empty) subset of $\mathbb{Z}^{p-d}$.

- If $\preceq$ denotes the inverse lexicographic order in $\mathbb{Z}^{p-d}$, then, for any $\underline{s}$ in $F$, we have $s \prec 0$.

- For each $\underline{s}$ in $F$, we have $\eta(\underline{s}) \in k^{*}$.

By (lemma 4.3.1,(4)), we have

$$
z_{l_{d}} u_{d+1}=\overline{h_{l_{d}}}\left(v_{1}\right) z_{l_{d}}^{b_{1}+1}+\cdots+\overline{h_{l_{d}}}\left(v_{M}\right) z_{l_{d}}^{b_{M}+1} .
$$

For any $\underline{e}=\left(e_{d+1}, \ldots, e_{p}\right) \in \mathbb{Z}^{p-d}$, let us denote $z^{\underline{e}}:=z_{l_{d+1}}^{e_{d+1}} \ldots z_{l_{p}}^{e_{p}}$.

Again by (lemma $4.3 .1,(4))$, there exists $\left(\lambda_{\underline{e}, 1}, \ldots, \lambda_{\underline{e}, M}\right) \in\left(k^{*}\right)^{M}$ such that

$$
z^{\underline{e}} u_{d+1}=\lambda_{\underline{e}, 1} v_{1} z_{l_{d}}^{b_{1}} z^{\underline{e}}+\cdots+\lambda_{\underline{e}, M} v_{M} z_{l_{d}}^{b_{M}} z^{\underline{e}} .
$$

Now, if we set $\underline{a}=\left(a_{d+1}, \ldots, a_{p}\right)$, we have

$$
x_{l_{d}}^{(m)} u=(1)+(2)
$$

with

$$
\begin{gathered}
(1)=z_{l_{d}} u_{d+1} z^{\underline{a}}=\overline{h_{l_{d}}}\left(v_{1}\right) z_{l_{d}+1}^{b_{1}+1} z^{\underline{a}}+\cdots+\overline{h_{l_{d}}}\left(v_{M}\right) z_{l_{d}}^{b_{M}+1} z^{\underline{a}} \\
=q^{-\left(\beta_{l_{d}}, \gamma_{d+1}+\beta_{j}-b_{1} \beta_{l_{d}}\right)} v_{1} z_{l_{d}}^{b_{1}+1} z^{\underline{a}}+\cdots+q^{-\left(\beta_{l_{d}}, \gamma_{d+1}+\beta_{j}-b_{M} \beta_{l_{d}}\right)} v_{M} z_{l_{d}}^{b_{M}+1} z^{\underline{a}}
\end{gathered}
$$

and

$$
(2)=\left(\sum_{\underline{s} \in F} \eta(\underline{s}) z^{\underline{s}}\right) u_{d+1} z^{\underline{a}}=\sum_{\underline{s} \in F} \eta(\underline{s})\left(\lambda_{\underline{s}, 1} v_{1} z_{l_{d}}^{b_{1}} z^{\underline{s}}+\cdots+\lambda_{\underline{s}, M} v_{M} z_{l_{d}}^{b_{M}} z^{\underline{s}}\right) z^{\underline{a}} .
$$


Thus, we also have

with

$$
u x_{l_{d}}^{(m)}=\left(1^{\prime}\right)+\left(2^{\prime}\right)
$$

$$
\begin{gathered}
\left(1^{\prime}\right)=u_{d+1} z^{\underline{a}} z_{l_{d}}=v_{1} z_{l_{d}}^{b_{1}} z^{\underline{a}} z_{l_{d}}+\cdots+v_{M} z_{l_{d}}^{b_{M}} z^{\underline{\underline{a}}} z_{l_{d}} \\
=q^{-\left(a_{d+1} \beta_{l_{d+1}}+\cdots+a_{p} \beta_{l_{p}}, \beta_{l_{d}}\right)}\left(v_{1} z_{l_{d}}^{b_{1}+1}+\cdots+v_{M} z_{l_{d}}^{b_{M}+1}\right) z^{\underline{a}}
\end{gathered}
$$

and

$$
\left(2^{\prime}\right)=u_{d+1} z^{\underline{a}}\left[\sum_{\underline{s} \in F} \eta(\underline{s}) z^{\underline{s}}\right]=\sum_{\underline{s} \in F} \eta(\underline{s})\left(v_{1} z_{l_{d}}^{b_{1}} z^{\underline{a}} z^{\underline{s}}+\cdots+v_{M} z_{l_{d}}^{b_{M}} z^{\underline{a}} z^{\underline{s}}\right) .
$$

Using lemma 4.4.4 and with $l=l_{d}$, we obtain:

$$
(1)+(2)=q^{\left(\beta_{l_{d}}, \beta_{m}-\beta_{j}\right)}\left[\left(1^{\prime}\right)+\left(2^{\prime}\right)\right] .
$$

We observe that (1) and $\left(1^{\prime}\right)$ are left linear combinations of monomials of type $z_{l_{d}}^{h} z^{\underline{a}}(h \in \mathbb{Z})$ with coefficients in $A=k\left\langle x_{j+1}^{\left(l_{d}\right)}, \ldots, x_{l_{d-1}}^{\left(l_{d}\right)}\right\rangle$, while $(2)$ and $\left(2^{\prime}\right)$ are left linear combinations of monomials of type $z_{l_{d}}^{h} z^{\underline{e}}(h \in \mathbb{Z}, \underline{e}=\underline{a}+\underline{s}, s \in F)$ with coefficients in $A$. Recall that, for any $\underline{s} \in F$, we have $\underline{s} \prec 0 \Rightarrow \underline{e}=\underline{a}+\underline{s} \prec \underline{a}$. So, by corollary 4.3 .2 , we have $(1)=q^{\left(\bar{\beta}_{d}, \beta_{m}-\beta_{j}\right)}\left(1^{\prime}\right)$, which implies that, if $1 \leq i$ $\leq M$, then

$$
q^{-\left(\beta_{l_{d}}, \gamma_{d+1}+\beta_{j}-b_{i} \beta_{l_{d}}\right)}=q^{\left(\beta_{l_{d}}, \beta_{m}-\beta_{j}\right)} q^{-\left(a_{d+1} \beta_{l_{d+1}}+\cdots+a_{p} \beta_{l_{p}}, \beta_{l_{d}}\right)} .
$$

Recall that, for $d+1 \leq h \leq p, \quad \gamma_{h}=s_{\beta_{l_{h}}}\left(\gamma_{h+1}\right)=\gamma_{h+1}-\left(\beta_{l_{h}}^{\vee}, \gamma_{h+1}\right) \beta_{l_{h}}$ $=\gamma_{h+1}-a_{h} \beta_{l_{h}}$. If we add up all these equalities, we obtain $\gamma_{d+1}=\gamma_{p+1}-$ $\left(a_{d+1} \beta_{l_{d+1}}+\cdots+a_{p} \beta_{l_{p}}\right)$. As $\gamma_{p+1}=\beta_{m}$, we get the formula

$$
-\left(\beta_{l_{d}}, \gamma_{d+1}+\beta_{j}-b_{i} \beta_{l_{d}}\right)=\left(\beta_{l_{d}}, \beta_{m}-\beta_{j}\right)-\left(\beta_{m}-\gamma_{d+1}, \beta_{l_{d}}\right)
$$

for any $i \in \llbracket 1, \ldots, M \rrbracket$.

This implies that $b_{i}\left\|\beta_{l_{d}}\right\|^{2}-\left(\beta_{l_{d}}, \gamma_{d+1}\right)=\left(\beta_{l_{d}}, \gamma_{d+1}\right)$. So, as the integers $b_{i}$ are distinct, we necessarily have $M=1, b_{1}=\left(\beta_{l_{d}}^{\vee}, \gamma_{d+1}\right)$ and $v_{1}$ is homogeneous of degree $\gamma_{d+1}-b_{1} \beta_{l_{d}}+\beta_{j}=\gamma_{d}+\beta_{j}$ if we set $\gamma_{d}=\gamma_{d+1}-b_{1} \beta_{l_{d}}=s_{\beta_{l_{d}}}\left(\gamma_{d+1}\right)$.

Now, in the case $d=c$, lemma 4.4 .7 becomes:

$$
u=u_{c} z_{l_{c}}^{a_{c}} \ldots z_{l_{p}}^{a_{p}}
$$

with:

- $u_{c} \in k^{*}$.

- If $\left(\gamma_{c}, \ldots, \gamma_{p}, \gamma_{p+1}\right)$ is the sequence of (not necessarily positive) roots recursively defined by $\gamma_{p+1}=\beta_{m}$ and, for $c \leq i \leq p, \gamma_{i}=s_{\beta_{l_{i}}}\left(\gamma_{i+1}\right)$, then each $a_{i}=\left(\beta_{l_{i}}^{\vee}, \gamma_{i+1}\right)$.

In this case, $u_{c}$ is homogeneous of degree 0 and, since each $z_{l_{d}}=x_{l_{d}}^{\left(l_{d}\right)}$ is homogeneous of degree $\beta_{l_{d}}$ (see lemma 4.3.1), we obtain that $u$ is also homogeneous of degree $a_{c} \beta_{l_{c}}+\cdots+a_{p} \beta_{l_{p}}$. So, as the degree of $u$ is uniquely defined, we have

$$
\beta_{m}+\beta_{j}=a_{c} \beta_{l_{c}}+\cdots+a_{p} \beta_{l_{p}}
$$

and, by proposition 2.3 .3 , we conclude:

Proposition 4.4.1. If $\mathcal{P}^{(m)}$ is not in $\operatorname{Im}\left(\phi_{m}\right)$, then

$$
\Delta=\left\{i \in \llbracket 1, \ldots, t \| \mid Z_{i} \in \mathcal{P}^{(2)}\right\}
$$

is not a positive diagram with respect to (1) in the sense of definition 2.2.2. 


\section{ConneCtions BetWeEn ADMissible AND POSITIVE DiAGRAMS}

Assume that $w$ and the algebra $R=U^{+}[w]$ are defined as in section 2. The admissible diagrams (with respect to the reduced expression (1)) are defined as in section 3.3.

We know that the longest element $w_{0}$ has a reduced expression of the form

$$
w_{0}=s_{\alpha_{1}} \ldots s_{\alpha_{N}}\left(\alpha_{i} \in \Pi \text { for } 1 \leq i \leq N=\left|\Phi^{+}\right|\right)
$$

such that $s_{\alpha_{1}} \ldots s_{\alpha_{t}}$ is the reduced expression (1) of $w$.

In this section, we shall use the following conventions:

- Denote by $R_{0}=U^{+}\left[w_{0}\right]$ the algebra associated to the reduced expression (17) as explained in section 2.1 and set $F_{0}=\operatorname{Fract}\left(R_{0}\right)$ its division ring of fractions.

- As in section 3.1, we set $X_{i}=X_{\beta_{i}}$ for $1 \leq i \leq N$ and we observe that $X_{1}$, $\ldots, X_{t}$ are also the generators of $R=U^{+}[w]$ defined in section 2.1, so that $R=k\left\langle X_{1}, \ldots, X_{t}\right\rangle$ is a subalgebra of $R_{0}=k\left\langle X_{1}, \ldots, X_{N}\right\rangle$.

- For any $\rho \in \mathbb{Z} \Pi$ we still denote by $h_{\rho}$ the automorphism of $R_{0}$ defined as in section 3.1 .

- Each $h_{\rho}$ can uniquely be extended to an automorphism, still denoted $h_{\rho}$, of $F_{0}$.

- We still denote by $H$ the set of all the automorphisms $h_{\rho}$ of $F_{0}(\rho \in \mathbb{Z} \Pi)$. It is a subgroup of $A u t\left(F_{0}\right)$.

- For any $m$ in $\llbracket 1, \ldots, N+1 \rrbracket$, we define the algebra $R_{0}^{(m)}$ and its canonical generators $X_{1}^{(m)}, \ldots, X_{N}^{(m)}$ as in section 3.2 .

5.1. A property of admissible diagrams. Consider $\Delta$ a diagram with respect to (11) (i.e. a subset of $\llbracket 1, \ldots, t \rrbracket)$. It is also a diagram with respect to (17) but it is not quite clear whether the properties " $\Delta$ is admissible with respect to (1)" and " $\Delta$ is admissible with respect to (17)" are equivalent. In this section, we clarify this point.

Lemma 5.1.1. (1) There exists a unique algebra isomorphism $g: R=$ $k\left\langle X_{1}, \ldots, X_{t}\right\rangle \rightarrow k\left\langle X_{1}^{(t+1)}, \ldots, X_{t}^{(t+1)}\right\rangle \subset R_{0}^{(t+1)}$ which transforms each $X_{i}(1 \leq i \leq t)$ into $X_{i}^{(t+1)}$.

(2) For any $\rho \in \mathbb{Z} \Pi$ and $B \in R$, we have $g \circ h_{\rho}(B)=h_{\rho} \circ g(B)$.

(3) The map $\mathcal{P} \mapsto g(\mathcal{P})$ is a bijection from the set of $H$-invariant prime ideals of $R$ onto the set of $H$-invariant prime ideals of $k\left\langle X_{1}^{(t+1)}, \ldots, X_{t}^{(t+1)}\right\rangle$.

Proof. (1) We know (see section 3.1) that $R$ is the $k$-algebra generated by the variables $X_{1}, \ldots, X_{t}$ submitted to the Levendorskii-Soibelman relations (10) and (11). As $X_{1}^{(t+1)}, \ldots, X_{t}^{(t+1)}$ satisfy the "same" relations (12) and (14) (see section 3.2), there exists a unique homomorphism $g: R=k\left\langle X_{1}, \ldots, X_{t}\right\rangle$ $\rightarrow k\left\langle X_{1}^{(t+1)}, \ldots, X_{t}^{(t+1)}\right\rangle \subset R_{0}^{(t+1)}$ which transforms each $X_{i}(1 \leq i \leq t)$ into $X_{i}^{(t+1)}$. As $g$ transforms the base of the ordered monomials $X_{1}, \ldots, X_{t}$ into the base of the ordered monomials in $X_{1}^{(t+1)}, \ldots, X_{t}^{(t+1)}$, it is an isomorphism.

(2) For $1 \leq i \leq t$, we have $g \circ h_{\rho}\left(X_{i}\right)=q^{-\left(\rho, \beta_{i}\right)} g\left(X_{i}\right)=q^{-\left(\rho, \beta_{i}\right)} X_{i}^{(t+1)}=h_{\rho}$ $\left(X_{i}^{(t+1)}\right)=h_{\rho} \circ g\left(X_{i}\right)$. This implies that the homomorphisms $g \circ h_{\rho}$ and $h_{\rho} \circ g$ coincide on $R$.

(3) Results thoroughly from (1) and (2). 
Until the end of this section, we identify the canonical generators $X_{1}, \ldots, X_{t}$ of $R$ with $X_{1}^{(t+1)}, \ldots, X_{t}^{(t+1)}$, respectively, so that $R$ is identified with the subalgebra $k\left\langle X_{1}^{(t+1)}, \ldots, X_{t}^{(t+1)}\right\rangle$ of $R_{0}^{(t+1)}, H$-Spec $(R)$ is identified with the set of $H$-invariant prime ideals of $k\left\langle X_{1}^{(t+1)}, \ldots, X_{t}^{(t+1)}\right\rangle$ and the division $\operatorname{ring} F=\operatorname{Fract}(R)$ is identified with the subalgebra of $F_{0}$ defined as follows: $F=\left\{a s^{-1} \mid a \in R, s \in R \backslash\{0\}\right\}$. ( $R$ and $F$ are both $H$-invariant.)

Lemma 5.1.2. Consider an integer $m$ with $2 \leq m \leq t+1$.

If $X_{1}^{(m)}, \ldots, X_{N}^{(m)}$ are the canonical generators of $R_{0}^{(m)}$, then $X_{1}^{(m)}, \ldots, X_{t}^{(m)}$ are the canonical generators of $R^{(m)}$.

Proof. We proceed by decreasing induction on $m$.

- If $m=t+1, X_{1}^{(t+1)}, \ldots, X_{t}^{(t+1)}$ are the canonical generators of $R$ by the previous identification. So, they also are the canonical generators of $R^{(t+1)}$ by the definition of this algebra.

- Assume that $2 \leq m \leq t$ and that $X_{1}^{(m+1)}, \ldots, X_{t}^{(m+1)}$ are the canonical generators of $R^{(m+1)}$. Denote (temporarily) by $X_{1}^{\prime(m)}, \ldots, X_{t}^{\prime(m)}$ the canonical generators of $R^{(m)}$. Recall (see section 3.2) that they are defined as follows:

- For each $i \in \llbracket 1, \ldots, t \rrbracket$, we have

$$
\begin{gathered}
\text { (1) } m \leq i \Rightarrow X_{i}^{\prime(m)}=X_{i}^{(m+1)} . \\
\text { (2) } i<m \Rightarrow X_{i}^{\prime(m)}=X_{i}^{(m+1)}+\sum_{l=1}^{+\infty} C_{l}^{(m+1)}\left(X_{m}^{(m+1)}\right)^{-l} \text { with } \\
C_{l}^{(m+1)}=\frac{\left(1-q_{m}\right)^{-l}}{[l] !_{q_{m}}} \lambda_{m, i}^{-l}\left(\delta_{m}^{(m+1)}\right)^{l}\left(X_{i}^{(m+1)}\right) .
\end{gathered}
$$

Moreover, if $h_{m}$ is the (unique) automorphism of $k\left\langle X_{1}^{(m+1)}, \ldots, X_{m-1}^{(m+1)}\right\rangle$ which satisfies $h_{m}\left(X_{i}^{(m+1)}\right)=\lambda_{m, i} X_{i}^{(m+1)}\left(\lambda_{m, i}=q^{-\left(\beta_{m}, \beta_{i}\right)}\right)$, we have $\delta_{m}^{(m+1)}(a)=$ $X_{m}^{(m+1)} a-h_{m}(a) X_{m}^{(m+1)}$ for any $a \in k\left\langle X_{1}^{(m+1)}, \ldots, X_{m-1}^{(m+1)}\right\rangle$. As $X_{1}^{(m)}, \ldots, X_{t}^{(m)}$ are defined by the same formulas, the proof is over.

Recall (see sections 3.1 and 3.2) that each prime ideal of $R_{0}^{(t+1)}$ (resp. $R$ ) is completely prime. So, if $\mathcal{Q}$ is any prime ideal of $R_{0}^{(t+1)}$, then $\mathcal{P}=\mathcal{Q} \cap R$ is a prime ideal of $R$. Moreover, since $R=k\left\langle X_{1}^{(t+1)}, \ldots, X_{t}^{(t+1)}\right\rangle$ is $H$-invariant, we have: ( $\mathcal{Q}$ is $H$-invariant $) \Rightarrow(\mathcal{P}$ is $H$-invariant $)$.

Lemma 5.1.3. If $\mathcal{P}$ is any $H$-invariant prime ideal of $R$, there exists a $H$ invariant prime ideal $\mathcal{Q}$ of $R_{0}^{(t+1)}$, such that

- $\mathcal{P}=\mathcal{Q} \cap R$.

- $\mathcal{Q} \cap\left\{X_{t+1}^{(t+1)}, \ldots, X_{N}^{(t+1)}\right\}=\emptyset$.

Proof. Observe that $R_{0}^{(t+1)}=R\left[X_{t+1}^{(t+1)} ; \sigma_{t+1}\right] \cdots\left[X_{N}^{(t+1)} ; \sigma_{N}\right]$, where each $\sigma_{i}$ is defined by the action of some element of $H$. So, lemma 5.1.3 actually follows easily from $N-t$ iterations of ([15], corollary 2.4).

Lemma 5.1.4. Consider $\mathcal{P}_{0} \in \operatorname{Spec}\left(R_{0}\right)$ and $\mathcal{P} \in \operatorname{Spec}(R)$.

Assume that $\mathcal{P}=\mathcal{Q} \cap R$ with $\mathcal{Q}=\mathcal{P}_{0}^{(t+1)}=$ the canonical image of $\mathcal{P}_{0}$ in $\operatorname{Spec}\left(R_{0}^{(t+1)}\right)$. 
For any $m \in \llbracket 2, \ldots, t+1 \rrbracket$, we denote by $\mathcal{P}^{(m)}\left(\right.$ resp. $\left.\mathcal{P}_{0}^{(m)}\right)$ the canonical image of $\mathcal{P}\left(\right.$ resp. $\left.\mathcal{P}_{0}\right)$ in $\operatorname{Spec}\left(R^{(m)}\right)$ (resp. Spec $\left(R_{0}^{(m)}\right)$ ) and we recall that, by lemma 5.1.2, $R^{(m)}$ is the subalgebra of $R_{0}^{(m)}$ generated by its $t$ first canonical generators $X_{1}^{(m)}, \ldots, X_{t}^{(m)}$. Then

$$
\mathcal{P}^{(m)}=\mathcal{P}_{0}^{(m)} \cap R^{(m)} .
$$

Proof. We prove this by decreasing induction on $m$.

- If $m=t+1$, we have $R^{(m)}=R, \mathcal{P}^{(m)}=\mathcal{P}$ and $\mathcal{P}_{0}^{(m)}=\mathcal{Q}$. So, we have the required equality by assumption.

- Assume $m \leq t$ and $\mathcal{P}^{(m+1)}=\mathcal{P}_{0}^{(m+1)} \cap R^{(m+1)}$.

- Assume that $X_{m}^{(m+1)} \notin \mathcal{P}_{0}^{(m+1)}$, so that $X_{m}^{(m+1)} \notin \mathcal{P}^{(m+1)}$.

Set $S_{m}=\left\{\left(X_{m}^{(m+1)}\right)^{d} \mid d \in \mathbb{N}\right\}$ and recall (sections 3.2 and 3.3) that

$* R^{(m)} S_{m}^{-1}=R^{(m+1)} S_{m}^{-1}$.

* $\mathcal{P}^{(m)}=\mathcal{P}^{(m+1)} S_{m}^{-1} \cap R^{(m)}$.

* $\mathcal{P}_{0}^{(m)}=\mathcal{P}_{0}^{(m+1)} S_{m}^{-1} \cap R_{0}^{(m)}$

As $R^{(m)} \subseteq R_{0}^{(m)}$ and $\mathcal{P}^{(m+1)} \subseteq \mathcal{P}_{0}^{(m+1)}$, we have

$\mathcal{P}^{(m)}=\mathcal{P}^{(m+1)} S_{m}^{-1} \cap R^{(m)} \subseteq \mathcal{P}_{0}^{(m+1)} S_{m}^{-1} \cap R_{0}^{(m)}=\mathcal{P}_{0}^{(m)}$.

This implies that

$$
\mathcal{P}^{(m)} \subseteq \mathcal{P}_{0}^{(m)} \cap R^{(m)} .
$$

Now, consider some $u \in \mathcal{P}_{0}^{(m)} \cap R^{(m)}$.

We have $\mathcal{P}_{0}^{(m)} \subseteq \mathcal{P}_{0}^{(m+1)} S_{m}^{-1} \Rightarrow u\left(X_{m}^{(m+1)}\right)^{d_{1}} \in \mathcal{P}_{0}^{(m+1)}$ with $d_{1} \in \mathbb{N}$.

We have $R^{(m)} \subseteq R^{(m+1)} S_{m}^{-1} \Rightarrow u\left(X_{m}^{(m+1)}\right)^{d_{2}} \in R^{(m+1)}$ with $d_{2} \in \mathbb{N}$.

Since $m \leq t, X_{m}^{(m+1)}$ is in $R^{(m+1)}$ and so, with $d=\max \left(d_{1}, d_{2}\right)$, we obtain that

$$
u\left(X_{m}^{(m+1)}\right)^{d} \in \mathcal{P}_{0}^{(m+1)} \cap R^{(m+1)}=\mathcal{P}^{(m+1)} \Rightarrow u \in \mathcal{P}^{(m+1)} S_{m}^{-1} \cap R^{(m)}=\mathcal{P}^{(m)} .
$$

This implies that

$$
\mathcal{P}^{(m)}=\mathcal{P}_{0}^{(m)} \cap R^{(m)} .
$$

- Assume that $X_{m}^{(m+1)} \in \mathcal{P}_{0}^{(m+1)}$, so that, since $m \leq t$,

$$
X_{m}^{(m+1)} \in \mathcal{P}_{0}^{(m+1)} \cap R^{(m+1)}=\mathcal{P}^{(m+1)} .
$$

Denote by

$$
g: R^{(m)} \rightarrow \frac{R^{(m+1)}}{\mathcal{P}^{(m+1)}}
$$

the only algebra homomorphism which transforms each $X_{i}^{(m)}(1 \leq$ $i \leq t$ ) into $g\left(X_{i}^{(m)}\right)=X_{i}^{(m+1)}+\mathcal{P}^{(m+1)}$ (the canonical image of $X_{i}^{(m+1)}$ in $\left.\frac{R^{(m+1)}}{\mathcal{P}^{(m+1)}}\right)$. We recall (section 3.3) that

$$
\mathcal{P}^{(m)}=\operatorname{ker}(g) .
$$

Denote by

$$
g_{0}: R_{0}^{(m)} \rightarrow \frac{R_{0}^{(m+1)}}{\mathcal{P}_{0}^{(m+1)}}
$$


the only algebra homomorphism which transforms each $X_{i}^{(m)}(1 \leq i$ $\leq N)$ into $g_{0}\left(X_{i}^{(m)}\right)=X_{i}^{(m+1)}+\mathcal{P}_{0}^{(m+1)}$ (the canonical image of $X_{i}^{(m+1)}$ in $\left.\frac{R_{0}^{(m+1)}}{\mathcal{P}_{0}^{(m+1)}}\right)$, so that

$$
\mathcal{P}_{0}^{(m)}=\operatorname{ker}\left(g_{0}\right)
$$

The canonical homomorphism $R_{0}^{(m+1)} \rightarrow \frac{R_{0}^{(m+1)}}{\mathcal{P}_{0}^{(m+1)}}$ restricted to $R^{(m+1)}$ has its kernel equal to $\mathcal{P}_{0}^{(m+1)} \cap R^{(m+1)}=\mathcal{P}^{(m+1)}$. So, it induces an injective homomorphism

$$
\epsilon: \frac{R^{(m+1)}}{\mathcal{P}^{(m+1)}} \rightarrow \frac{R_{0}^{(m+1)}}{\mathcal{P}_{0}^{(m+1)}}
$$

which transforms each $X_{i}^{(m+1)}+\mathcal{P}^{(m+1)}(1 \leq i \leq t)$ into $X_{i}^{(m+1)}+$ $\mathcal{P}_{0}^{(m+1)}$. So,

$$
\epsilon \circ g: R^{(m)} \rightarrow \frac{R_{0}^{(m+1)}}{\mathcal{P}_{0}^{(m+1)}}
$$

is an algebra homomorphism which transforms each $X_{i}^{(m)}(1 \leq i \leq t)$ into $X_{i}^{(m+1)}+\mathcal{P}_{0}^{(m+1)}=g_{0}\left(X_{i}^{(m)}\right)$. This implies that $\epsilon \circ g$ is the restriction of $g_{0}$ to $R^{(m)}$ and so, $\operatorname{Ker}(\epsilon \circ g)=\mathcal{P}_{0}^{(m)} \cap R^{(m)}$. Since $\epsilon$ is injective, we also have $\operatorname{Ker}(\epsilon \circ g)=\operatorname{Ker}(g)=\mathcal{P}^{(m)}$ and the proof is over.

Proposition 5.1.1. Consider a diagram $\Delta$ with respect to (1) (i.e. a subset of $\llbracket 1, \ldots, t \rrbracket)$, so that $\Delta$ is also a diagram with respect to (17)). Then $\Delta$ is admissible with respect to (11) if and only if $\Delta$ is admissible with respect to (17).

Proof. - Assume that $\Delta$ is admissible with respect to (17). This means (see section 3.3) that there exists some $\mathcal{P}_{0}$ in $\operatorname{Spec}\left(R_{0}\right)$ such that its canonical image $\mathcal{P}_{0}^{(2)}$ in $\operatorname{Spec}\left(R_{0}^{(2)}\right)$ satisfies:

$$
\mathcal{P}_{0}^{(2)} \cap\left\{Z_{1}, \ldots, Z_{N}\right\}=\left\{Z_{i} \mid i \in \Delta\right\} .
$$

Denote by $\mathcal{Q}=\mathcal{P}_{0}^{(t+1)}$ the canonical image of $\mathcal{P}_{0}$ in $\operatorname{Spec}\left(R_{0}^{(t+1)}\right)$ and set $\mathcal{P}=\mathcal{Q} \cap R$. We know that $\mathcal{P} \in \operatorname{Spec}(R)$ and, by lemma 5.1.4, its canonical image $\mathcal{P}^{(2)}$ in $\operatorname{Spec}\left(R^{(2)}\right)$ satisfies

$$
\mathcal{P}^{(2)}=\mathcal{P}_{0}^{(2)} \cap R^{(2)} .
$$

So,

$$
\mathcal{P}^{(2)} \cap\left\{Z_{1}, \ldots, Z_{t}\right\}=\mathcal{P}_{0}^{(2)} \cap R^{(2)} \cap\left\{Z_{1}, \ldots, Z_{t}\right\}=\mathcal{P}_{0}^{(2)} \cap\left\{Z_{1}, \ldots, Z_{t}\right\} .
$$

Since $\Delta$ is a subset of $\llbracket 1, \ldots, t \rrbracket)$, this implies that

$$
\mathcal{P}^{(2)} \cap\left\{Z_{1}, \ldots, Z_{t}\right\}=\left\{Z_{i} \mid i \in \Delta\right\} .
$$

So $\Delta$ is admissible with respect to (11).

- Assume that $\Delta$ is admissible with respect to (1). This implies (see section 3.3) that there exists some $\mathcal{P}$ in $H-\operatorname{Spec}(R)$ whose canonical image $\mathcal{P}^{(2)}$ in $\operatorname{Spec}\left(R^{(2)}\right)$ is the ideal of $R^{(2)}$ generated by $\left\{Z_{i} \mid i \in \Delta\right\}\left(\mathcal{P}^{(2)}=0\right.$ if $\Delta=\emptyset)$. 
Since $\mathcal{P}$ is $H$-invariant, it follows from lemma 5.1.3 that there exists a $(H$ invariant) prime ideal $\mathcal{Q}$ of $R_{0}^{(t+1)}$, such that

$-\mathcal{P}=\mathcal{Q} \cap R$.

$-\mathcal{Q} \cap\left\{X_{t+1}^{(t+1)}, \ldots, X_{N}^{(t+1)}\right\}=\emptyset$.

Now, by corollary 4.1.1, there exists $\mathcal{P}_{0}$ in $\operatorname{Spec}\left(R_{0}\right)$ whose canonical image $\mathcal{P}_{0}^{(t+1)}$ in $\operatorname{Spec}\left(R_{0}^{(t+1)}\right)$ is equal to $\mathcal{Q}$ and whose canonical image $\mathcal{P}_{0}^{(2)}$ in $\operatorname{Spec}\left(R_{0}^{(2)}\right)$ does not meet the set $\left\{Z_{t+1}, \ldots, Z_{N}\right\}$.

As above, by lemma 5.1.4, we have

$$
\mathcal{P}^{(2)}=\mathcal{P}_{0}^{(2)} \cap R^{(2)} .
$$

Since $\mathcal{P}_{0}^{(2)}$ does not meet the set $\left\{Z_{t+1}, \ldots, Z_{N}\right\}$, we have

$$
\mathcal{P}_{0}^{(2)} \cap\left\{Z_{1}, \ldots, Z_{N}\right\}=\mathcal{P}_{0}^{(2)} \cap\left\{Z_{1}, \ldots, Z_{t}\right\} .
$$

So, since $\left\{Z_{1}, \ldots, Z_{t}\right\} \subseteq R^{(2)}$, we can write

$$
\begin{gathered}
\mathcal{P}_{0}^{(2)} \cap\left\{Z_{1}, \ldots, Z_{N}\right\}=\mathcal{P}_{0}^{(2)} \cap R^{(2)} \cap\left\{Z_{1}, \ldots, Z_{t}\right\} \\
=\mathcal{P}^{(2)} \cap\left\{Z_{1}, \ldots, Z_{N}\right\}=\left\{Z_{i} \mid i \in \Delta\right\} .
\end{gathered}
$$

This proves that $\Delta$ is admissible with respect to (17).

\subsection{A partial result.}

Lemma 5.2.1. For any diagram $\Delta$ (with respect to (1)) we have:

$$
\Delta \text { positive } \Rightarrow \Delta \text { admissible. }
$$

Proof. As in section 3.3, we denote by $\mathcal{P}_{\Delta}^{(2)}$ the ideal of $\bar{R}=R^{(2)}$ generated by the canonical generators $Z_{i}$ with $i \in \Delta\left(\mathcal{P}_{\Delta}^{(2)}=0\right.$ if $\left.\Delta=\emptyset\right)$. Recall (see section 3.3) that $\mathcal{P}_{\Delta}^{(2)} \in H-\operatorname{Spec}\left(R^{(2)}\right)$.

Consider an integer $m$ with $2 \leq m \leq t+1$. We first prove, by induction on $m$, that there exists a prime ideal $\mathcal{P}^{(m)} \in \operatorname{Spec}\left(R^{(m)}\right)$ such that $\mathcal{P}_{\Delta}^{(2)}$ is the canonical image of $\mathcal{P}^{(m)}$ in $\operatorname{Spec}\left(R^{(2)}\right)$.

- If $m=2$, we just have to choose $\mathcal{P}^{(2)}=\mathcal{P}_{\Delta}^{(2)}$.

- Assume that, for some $m$ with $2 \leq m \leq t$, there exists a prime ideal $\mathcal{P}^{(m)} \in \operatorname{Spec}\left(R^{(m)}\right)$ such that $\mathcal{P}_{\Delta}^{(2)}$ is the canonical image of $\mathcal{P}^{(m)}$ in $\operatorname{Spec}\left(R^{(2)}\right)$. Since $\Delta$ is positive, we deduce from proposition 4.4.1 that $\mathcal{P}^{(m)}=\phi_{m}\left(\mathcal{P}^{(m+1)}\right)$ with $\mathcal{P}^{(m+1)}$ in $\operatorname{Spec}\left(R^{(m+1)}\right)$.

So, $\mathcal{P}_{\Delta}^{(2)}$ is the canonical image of $\mathcal{P}^{(m+1)}$ in $\operatorname{Spec}\left(R^{(2)}\right)$, and our affirmation is proved.

In particular, there exists $\mathcal{P} \in \operatorname{Spec}\left(R^{(t+1)}\right)=\operatorname{Spec}(R)$ such that $\mathcal{P}_{\Delta}^{(2)}$ is the canonical image of $\mathcal{P}$ in $\operatorname{Spec}\left(R^{(2)}\right)$. This means (see section 3.3) that $\Delta$ is admissible.

Proposition 5.2.1. Assume that $w=w_{0}$. Then, the positive diagrams (with respect to (1) coincide with the admissible diagrams (with respect to (1D)).

Proof. By lemma 5.2.1, the set of positive diagrams is contained in the set of admissible diagrams. By proposition 2.3.1, the number of positive diagrams is equal to the number of $u$ in the Weyl group $W$ such that $u \leq w$ (where $\leq$ denotes 
the Bruhat order). Since we assume that $w=w_{0}$, we get that the number of positive diagrams is equal to the cardinal $|W|$ of $W$. Since the set of admissible diagrams is in one-to-one correspondence with $H-\operatorname{Spec}(R)=H-\operatorname{Spec}\left(U^{+}\right)$, the number of admissible diagrams does not depend on the reduced decomposition of $w$. In [19, Antoine Mériaux gives a precise description of admissible diagrams, for each type of the simple complex Lie algebra $\mathfrak{g}$, and for a particular reduced decomposition of $w_{0}$ so that he can compute their number and check that it is precisely equal to $|W|$. This implies that the positive diagrams coincide with the admissible diagrams.

Let us observe that, in this proof, we use a result of Antoine Mériaux to prove that the number of admissible diagrams (which is also the cardinal of $H-\operatorname{Spec}\left(U^{+}\right)$) is equal to $|W|$. This equality can also be obtained using results of M. Gorelik, N. Andruskiewitsch and F. Dumas ([9] and [2]) or T. J. Hodges and T. Levasseur and M. Toro [11, but this should require some (minor) restricted assumptions on the choice of the ground field $k(\operatorname{char}(k)=0)$ or on the parameter $q$ ( $q$ transcendental).

5.3. The general case. We can now prove, in the general case (i.e. $w$ is no longer assumed to be the longest element of $W$ ):

Theorem 5.3.1. The positive diagrams (with respect to (11) coincide with the admissible diagrams (with respect to (11)).

Proof. Consider a diagram $\Delta$ with respect to (11) (i.e. a subset of $\llbracket 1, \ldots, t \rrbracket$ ), so that $\Delta$ is also a diagram with respect to (17).

- Assume that $\Delta$ is positive (with respect to (1)). By proposition 2.3.2, $\Delta$ is positive with respect to (17) and, by proposition 5.2.1, $\Delta$ is admissible with respect to (17). Now, by proposition $5.1 .1, \Delta$ is admissible with respect to (1).

- Assume that $\Delta$ is admissible (with respect to (10)). By proposition 5.1.1, $\Delta$ is admissible with respect to (17) and, by proposition $5.2 .1, \Delta$ is positive with respect to (17). Now, by proposition $2.3 .2, \Delta$ is positive with respect to (1).

Corollary 5.3.1. (All the diagrams are with respect to (11).)

(1) The map $\zeta: \Delta=\left\{j_{1}, \ldots, j_{s}\right\} \mapsto u=w^{\Delta}=s_{\alpha_{j_{1}}} \circ \cdots \circ s_{\alpha_{j_{s}}}$ is a bijection from the set of admissible diagrams onto the set $\{u \in W \mid u \leq w\}(\zeta(\emptyset)=I d)$.

(2) Consider an admissible diagram $\Delta=\left\{j_{1}, \ldots, j_{s}\right\}$ and some integer $i \in$ $\llbracket 1, \ldots, t \rrbracket$. Set $\Delta \cap \llbracket i+1, \ldots, t \rrbracket=\left\{j_{c}, \ldots, j_{s}\right\}(1 \leq c \leq s)$. Then the expression $s_{\alpha_{i}} \circ s_{\alpha_{j_{c}}} \circ \cdots \circ s_{\alpha_{j_{s}}}$ is reduced. In particular, $s_{\alpha_{j_{1}}} \circ \cdots \circ s_{\alpha_{j_{s}}}$ is a reduced expression of $\zeta(\Delta)$.

(3) Consider some $u \in W$ with $u \leq w$. Then, the only admissible diagram $\Delta$ such that $\zeta(\Delta)=u$ is recursively defined as follows:

- $1 \in \Delta \Leftrightarrow l\left(s_{\alpha_{1}} \circ u\right)=l(u)-1 \Leftrightarrow u^{-1}\left(\alpha_{1}\right)$ is a negative root.

- Consider some integer $i \in \llbracket 1, \ldots, t-1 \rrbracket$ and assume that $\Delta \cap \llbracket 1, \ldots, i \rrbracket$ $=\left\{j_{1}, \ldots, j_{d}\right\}$.

Set $u_{i}=s_{\alpha_{j_{d}}} \circ \cdots \circ s_{\alpha_{j_{2}}} \circ s_{\alpha_{j_{1}}} \circ u\left(u_{i}=u\right.$ if $\left.\Delta \cap \llbracket 1, \ldots, i \rrbracket=\emptyset\right)$. Then $i+1 \in \Delta \Leftrightarrow l\left(s_{\alpha_{i+1}} \circ u_{i}\right)=l\left(u_{i}\right)-1 \Leftrightarrow u_{i}^{-1}\left(\alpha_{i+1}\right)$ is a negative root.

Proof. As the admissible diagrams coincide with the positive diagrams, (1) is proposition 2.3.1 (assertion (2)) and (2) is proposition 2.2.2. Now, let us prove (3). 
- Assume that $1 \in \Delta$, so that $\Delta=\left\{j_{1}, \ldots, j_{s}\right\}$ with $j_{1}=1$. As $u=\zeta(\Delta)=$ $s_{\alpha_{j_{1}}} \circ \cdots \circ s_{\alpha_{j_{s}}}$, we have $l\left(s_{\alpha_{1}} \circ u\right)=l\left(s_{\alpha_{j_{2}}} \circ \cdots \circ s_{\alpha_{j_{s}}}\right)=s-1=l(u)-1$.

Conversely, assume that $l\left(s_{\alpha_{1}} \circ u\right)=l(u)-1$. If $1 \notin \Delta$, then $\Delta \cap \llbracket 2, \ldots, t \rrbracket=\Delta$ and, by $(2), l\left(s_{\alpha_{1}} \circ u\right)=l(u)+1$. So, we obtain a contradiction.

Now, we have classically: $l\left(s_{\alpha_{1}} \circ u\right)=l(u)-1 \Leftrightarrow l\left(u^{-1} \circ s_{\alpha_{1}}\right)=l\left(u^{-1}\right)-1 \Leftrightarrow$ $u^{-1}\left(\alpha_{1}\right)$ is a negative root.

- Assume that $i+1 \in \Delta$, so that $\Delta \cap \llbracket i+1, \ldots, t \rrbracket=\left\{j_{d+1}, \ldots, j_{s}\right\}$ with $j_{d+1}=$ $i+1$. As $u_{i}=s_{\alpha_{j_{d}}} \circ \cdots \circ s_{\alpha_{j_{2}}} \circ s_{\alpha_{j_{1}}} \circ u=s_{\alpha_{j_{d+1}}} \circ \cdots \circ s_{\alpha_{j_{s}}}$, we have $l\left(s_{\alpha_{i+1}} \circ\right.$ $u)=l\left(s_{\alpha_{j_{d+2}}} \circ \cdots \circ s_{\alpha_{j_{s}}}\right)=s-(d+1)=l\left(u_{i}\right)-1$.

Conversely, assume that $l\left(s_{\alpha_{i+1}} \circ u_{i}\right)=l\left(u_{i}\right)-1$. If $i+1 \notin \Delta$, then $\Delta \cap \llbracket i+$ $2, \ldots, t \rrbracket=\left\{j_{d+1}, \ldots, j_{s}\right\}$ and, by $(2), l\left(s_{\alpha_{i+1}} \circ u_{i}\right)=l\left(u_{i}\right)+1$. So, we still have a contradiction and we conclude as above.

From corollary 5.3.1 (assertions (1) and (2)) and lemma 2.2.1 (assertion (2)), we deduce:

Corollary 5.3.2. (1) The map $\zeta^{\prime}: \Delta=\left\{j_{1}, \ldots, j_{s}\right\} \mapsto u^{\prime}=v^{\Delta}=s_{\alpha_{j_{s}}} \circ$ $\cdots \circ s_{\alpha_{j_{1}}}$ is a bijection from the set of admissible diagrams onto the set $\left\{u \in W \mid u \leq v=w^{-1}\right\}\left(\zeta^{\prime}(\Delta)=(\zeta(\Delta))^{-1}, \zeta^{\prime}(\emptyset)=I d\right)$.

(2) Consider an admissible diagram $\Delta=\left\{j_{1}, \ldots, j_{s}\right\}$ and some integer $i \in$ $\llbracket 1, \ldots, t \rrbracket$. Set $\Delta \cap \llbracket i+1, \ldots, t \rrbracket=\left\{j_{c}, \ldots, j_{s}\right\}(1 \leq c \leq s)$. Then the expression $s_{\alpha_{j_{s}}} \circ \cdots \circ s_{\alpha_{j_{c}}} \circ s_{\alpha_{i}}$ is reduced. In particular, $s_{\alpha_{j_{s}}} \circ \cdots \circ s_{\alpha_{j_{1}}}$ is a reduced expression of $\zeta^{\prime}(\Delta)$.

If $w$ and its reduced decomposition are chosen as in the example of section 2.1, we know (proposition 2.1.1) that $U^{+}[w]$ is the algebra of quantum matrices $O_{q}\left(M_{p, m}(k)\right)$ with $m=n-p+1$ and with the same canonical generators (as $\left.U^{+}[w]\right)$. So, by [5], the admissible diagrams are the I-diagrams. In this case, corollary 5.3.2 has also been proved (with quite different methods) by A. Postnikov ([20, theorem 19.1) and by T. Lam and L. Williams ([17, theorem 5.3).

In the general case, using the bijection between the set of admissible diagrams and $H-\operatorname{Spec}\left(U^{+}[w]\right)$ recalled in section 3.3, the two bijections of proposition 2.3.1 and the coincidence of admissible and positive diagrams (theorem 5.3.1), we get

Corollary 5.3.3. (1) There exists a natural bijection from the set $\{u \in W \mid u \leq$ $\left.v=w^{-1}\right\}$ onto $H-\operatorname{Spec}\left(U^{+}[w]\right)$. It is defined by

$$
v^{\Delta} \mapsto \mathcal{P}_{\Delta}
$$

where $\Delta$ runs through the set of admissible diagrams.

(2) There exists a natural bijection from the set $\{u \in W \mid u \leq w\}$ onto $H-$ $\operatorname{Spec}\left(U^{+}[w]\right)$. It is defined by

$$
w^{\Delta} \mapsto \mathcal{P}_{\Delta}
$$

where $\Delta$ runs through the set of admissible diagrams.

When $U^{+}[w]$ is the quantum matrix algebra $O_{q}\left(M_{p, m}(k)\right)$ as above, S. Launois 14] has constructed (with different methods) a bijection from $\{u \in W \mid u \leq v\}$ onto $H-\operatorname{Spec}\left(O_{q}\left(M_{p, m}(k)\right)\right)$ which, moreover, preserves the ordering (where the Weyl group is provided with the Bruhat order and $H-\operatorname{Spec}\left(O_{q}\left(M_{p, m}(k)\right)\right)$ is provided with the inclusion). So, it seems natural to ask the following questions: 
Question 5.3.1. Assume $w$ and its reduced decomposition chosen as in the example of section 2.1.

Does the S. Launois bijection from $\{u \in W \mid u \leq v\}$ onto $H-\operatorname{Spec}\left(O_{q}\left(M_{p, m}(k)\right)\right)$ $=H-\operatorname{Spec}\left(U^{+}[w]\right)$ coincide with the first bijection of corollary 5.3.3? If not, is there a simple relation between those two bijections?

Question 5.3.2. In the general case, are the two bijections of corollary 5.3 .3 isomorphisms of ordered sets, where the set $\{u \in W \mid u \leq v\}$, (resp. $\{u \in W \mid u \leq w\}$ ) is provided with the Bruhat order and $H-\operatorname{Spec}\left(U^{+}[w]\right)$ is provided with the inclusion?

Under stricter assumptions than in the present paper (namely assuming that $k$ has characteristic zero and that $q$ is transcendental over $\mathbb{Q}$ ), M. Yakimov 22 constructs an order isomorphism between the set $\{u \in W \mid u \leq w\}$ equipped with the Bruhat order and $H-\operatorname{Spec}\left(U^{+}[w]\right)$ equipped with inclusion. This raises a new question:

Question 5.3.3. Is this Yakimov bijection the same as the one in the corollary $5.3 .3(2)$ ?

\section{REFERENCES}

[1] J. Alev and F. Dumas, Sur le corps des fractions de certaines algèbres quantiques, J. Algebra 170 (1994), 229-265. MR1302839 (96c:16033)

[2] N. Andruskiewitsch and F. Dumas, On the automorphisms of $U_{q}^{+}(\mathfrak{g})$ "Quantum Groups" (B. Enriquez, ed.), IRMA Lectures in Mathematical and Theoretical Physics 12 European Math. Society (2008), 107-133. MR2432991(2009i:17019)

[3] K. A. Brown and K. R. Goodearl, Lectures on algebraic quantum groups, Advanced Courses in Mathematics, CRM Barcelona, Birkhäuser Verlag, Basel, 2002. MR.1898492 (2003f:16067)

[4] G. Cauchon, Effacement des dérivations et spectres premiers des algèbres quantiques, J. Algebra 260 (2003), 476-518. MR:1967309 (2004g:16044)

[5] G. Cauchon, Spectre premier de $O_{q}\left(M_{n}(k)\right.$ : image canonique et séparation normale. J. Algebra 260 (2003), 419-569. MR1967310 (2004g:16045)

[6] V. Deodhar, A combinatorial setting for questions in Kazhdan-Lusztig theory, Geometriae Dedicata 36 (1990), 95-119. MR.1065215 (91h:20075)

[7] K. R. Goodearl and E. S. Letzter, Prime factor algebras of the coordinate ring of quantum matrices, Proc. Amer. Math. Soc. 121 (1994), 1017-1025. MR.1211579 (94j:16066)

[8] K. R. Goodearl and R. B. Warfield Jr., An introduction to noncommutative notherian rings, London Math. Soc. Student texts 16, Cambridge University Press, Cambridge (1989). MR.1020298 (91c:16001)

[9] M. Gorelik, The prime and primitive spectra of a quantum Bruhat cell translate, J. Algebra 227 (2000), 211-253. MR.1754232 (2001d:17012)

[10] J. E. Humphreys, Introduction to Lie Algebras and Representations Theory, Graduate Texts in Mathematics 9, Springer-Verlag, New York (1980). MR499562 (81b:17007)

[11] T. J. Hodges and T. Levasseur and M. Toro, Algebraic structure of multiparameter quantum groups, Advances in Maths. 126 (1997), 52-92. MR.1440253 (98e:17022)

[12] J. C. Jantzen, Lectures on quantum groups, Graduate Studies in Mathematics 6, Amer. Math. Soc., Providence (1996). MR.1359532 (96m:17029)

[13] A. Joseph, Quantum Groups and Their Primitive Ideals, Ergebnisse der Mathematik (3) 29, Springer-Verlag, Berlin (1995). MR1315966 (96d:17015)

[14] S. Launois, Combinatorics of H-primes in quantum matrices, J. Algebra 309 (2007), 139-167. MR2301235 (2007k:05221)

[15] S. Launois, T. H. Lenagan and L. Rigal, Quantum unique factorisation domains, J. London Math. Soc. (2) 74 (2006), 321-340. MR2269632(2007h:16047)

[16] S. Z. Levendorskii and Ya. S. Soibelman, Algebras of functions on a compact quantum group, Schubert cells and quantum tori, Comm. Math. Phys. 139 (1991), 141-170. MR.1116413 (92h:58020) 
[17] T. Lam and L. Williams, Total positivity for cominuscule grassmannians, New York Journal of Mathematics 14 (2008), 53-99. MR.2383586 (2008m:05307)

[18] R. Marsh and K. Rietsch, Parametrisations of flag varieties, Representation Theory, 8 (2004), 212-242. MR2058727 (2005c:14061)

[19] A. Mériaux, Les diagrammes de Cauchon pour $U_{q}^{+}(\mathfrak{g})$, J. Algebra 323 (2010), 1060-1097.

[20] A. Postnikov, Total positivity, Grassmannians and Networks, Preprint, 2006: http:// arxiv.org/abs/math/0609764.

[21] M. Yakimov, Invariant prime ideals in quantizations of nilpotent Lie algebras, Preprint, 2009: http://arxiv.org/abs/0905.0852

[22] H. Yamane, A Poincaré-Birkhoff-Witt theorem for quantized universal enveloping algebra of type $A_{N}$, Publ. Res. Inst. Math. Sci. 25 (1989), 503-520. MR1018513 (91a:17016)

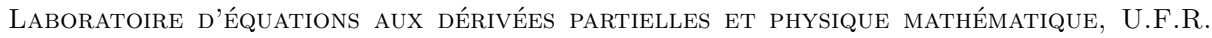
Sciences, B.P. 1039, 51687 Reims Cedex 2, France.

E-mail address: antoine.meriaux@univ-reims.fr

Laboratoire D'Équations auX dérivées Partielles et Physique mathématique, U.F.R. Sciences, B.P. 1039, 51687 Reims Cedex 2, France

E-mail address: gerard.cauchon@univ-reims.fr 